\title{
OSCILLATIONS FORTES SUR UN CHAMP LINÉAIREMENT DÉGÉNÉRÉ
}

\author{
PAr Christophe CHEVERry, Olivier GUÈS et Guy MÉtIVIER
}

\begin{abstract}
RÉSUMÉ. - On s'intéresse à des systèmes quasilinéaires en multidimension d'espace. En présence de valeurs propres linéairement dégénérées, des transparences se produisent. Les calculs classiques d'optique géométrique conduisent à des équations qui sont linéaires. On montre dans cet article que les amplitudes des développements asymptotiques peuvent être augmentées pour atteindre des régimes non linéaires sans pour autant compromettre la construction de solutions approchées. On met en valeur les contraintes permettant de justifier l'existence de solutions exactes qui correspondent à de telles solutions approchées. En l'absence de ces contraintes, on classifie les différents types d'instabilité créés.
\end{abstract}

(C) 2003 Published by Elsevier SAS

ABSTRACT. - Transparencies happen for quasilinear waves associated to linearly degenerate eigenvalues, leading to linear geometric optics. To reach nonlinear phenomena, we have to consider larger amplitudes. We show that approximate solutions can still be constructed. We find intrinsic conditions allowing to justify the approximation. In the absence of these conditions, instabilities are created. We analyse the different types of the underlying instabilities.

ㄷ 2003 Published by Elsevier SAS

\section{Introduction}

Des résultats généraux ont vu le jour ces dernières années en matière d'optique géométrique non linéaire (voir par exemple [17]). Lorsque les équations possèdent des structures particulières, comme c'est souvent le cas pour les modèles issus de la physique, les équations de transport, au lieu d'être non linéaires, sont linéaires. C'est le phénomène appelé transparence dans [15]. Pour atteindre des régimes non linéaires, on doit soit augmenter les temps (ou distances) de propagation, soit augmenter les amplitudes au-delà des régimes considérés dans les théorèmes généraux.

Pour des systèmes semi-linéaires, ce programme a connu de sérieuses avancées, cf. les travaux de Joly, Métivier et Rauch [15] sur les équations de Maxwell-Bloch, de Colin-Lannes [5] pour la mécanique des fluides, et de Jeanne [14] pour les équations de Yang-Mills.

Pour les systèmes de lois de conservation, l'équation de propagation générale pour les enveloppes de paquets d'onde est de la forme :

$$
\partial_{t} u+c \partial_{x} u+\gamma \partial_{\theta} u^{2}=0 .
$$

Le coefficient d'auto-interaction $\gamma$ s'annule exactement quand l'oscillation est associée à un mode linéairement dégénéré. La plupart des systèmes physiques ont des valeurs propres linéairement dégénérées et la question de l'optique géométrique transparente est donc extrêmement 
naturelle. Le but de cet article est d'aborder ce problème, dans le contexte des systèmes multidimensionnels.

On se place en dimension $d$ d'espace et on considère le système

$$
\partial_{t} f_{0}(u)+\sum_{j=1}^{d} \partial_{x_{j}} f_{j}(u)=0
$$

où les flux $f_{j}$ sont définis et $C^{\infty}$ sur un ouvert $\mathcal{U} \subset \mathbb{R}^{N}$, et à valeurs dans $\mathbb{R}^{N}$. On suppose que ce système admet une entropie strictement convexe. On se donne une solution $\bar{u}_{0}$ de l'équation (1.1). On cherche des familles de solutions paramétrées par $\varepsilon \in] 0,1]$, ayant un développement asymptotique de la forme :

$$
u^{\varepsilon}(t, x) \underset{\varepsilon \rightarrow 0}{\sim} \dot{u}_{a}^{\varepsilon}(t, x)=\bar{u}_{0}(t, x)+\sum_{n=n_{0}}^{n_{1}}(\sqrt{\varepsilon})^{n} u_{n}\left(t, x, \frac{\varphi_{\varepsilon}(t, x)}{\varepsilon}\right)
$$

où $n_{0} \leqslant n_{1}$ sont deux entiers. Le paramètre $\varepsilon$ joue le rôle d'une longueur d'onde. Le terme principal des oscillations est d'amplitude en $\mathrm{O}\left(\varepsilon^{n_{0} / 2}\right)$ pour une fréquence en $\mathrm{O}\left(\varepsilon^{-1}\right)$. Le cas $n_{0}=2$ est le régime général de l'optique géométrique dite faiblement non linéaire. Pour $n_{0}=1$, on parlera d'oscillations fortes. Pour $n_{0}=0$, on atteint le domaine des oscillations de grande amplitude.

Pour un système quelconque, les oscillations de faible amplitude $\left(n_{0}=2\right)$ se propagent sur n'importe quel mode. Ce résultat est établi dans les articles [11,12,18]. Appliqué aux modes associés à des valeurs propres linéairement dégénérées, il conduit à des équations de transport qui sont linéaires, donnant lieu au phénomène de transparence. Dans le cas d'ocillations associées à des modes linéairement dégénérés, on s'attend alors à pouvoir augmenter la force des oscillations.

En dimension un d'espace, le problème de l'existence et la stabilité d'oscillations de grande amplitude $\left(n_{0}=0\right)$ associées à des modes linéairement dégénérés a été étudié par W. $\mathrm{E}$ [7] pour la dynamique des gaz 1D, puis par A. Heibig [13] et B. Sévennec [24] dans un cadre général. Ces contributions sont aujourd'hui englobées dans un énoncé obtenu par A. Corli et O. Guès [6], puis précisé par A. Museux [20], et qui se situe dans le cadre plus général des solutions stratifiées.

Par ailleurs, en dimension quelconque, associées à des modes linéairement dégénérés, on dispose toujours de solutions particulières de (1.1) sous la forme d'ondes simples

$$
u(t, x)=v(h(\xi \cdot x-\sigma t))
$$

avec $v$ de classe $C^{1}$ sur un intervalle $I \subset \mathbb{R}, \xi$ et $\sigma$ bien choisis et $h$ une fonction arbitraire de $C^{1}(\mathbb{R} ; I)$ (cf. [19]). On peut choisir $h$ périodique de période $\varepsilon$, obtenant ainsi des oscillations de grande amplitude associées à la phase $\varphi=\xi \cdot x-\sigma t$. La question est alors d'étudier la stabilité de telles solutions.

Dans ce travail, on aborde de façon systématique en dimension $d>1$ la construction de solutions ayant un développement asymptotique de la forme (1.2), avec $n_{0}=1$. Les résultats vont principalement dans deux directions.

- En premier lieu, on montre que, en général, le régime pertinent de l'optique géométrique associée à un champ linéairement dégénéré est celui des oscillations fortes. Pour cela, par une méthode BKW, on établit l'existence de solutions approchées (1.2) avec $n_{0}=1$, polarisées sur un mode linéairement dégénéré (théorème 3.1). Ce sont les conditions de transparence résultant de l'hypothèse de dégénérescence linéaire, qui permettent de dévisser la cascade des 
équations $\mathrm{BKW}$ en une suite de problèmes bien posés résolus par récurrence. Le régime des oscillations fortes donne donc toujours lieu à des équations BKW bien posées, au contraire de celui des oscillations de grandes amplitudes comme montré par D. Serre [22] dans le cadre de la dynamique des gaz isentropiques, pour les oscillations de grande amplitude polarisées sur la vitesse.

D'autre part, l'évolution du profil principal $u_{1}^{*}$ est en général couplée à celle de la moyenne $\left\langle u_{2}\right\rangle$. Le système $\left(\mathcal{Z}_{1}\right)$ qui gouverne cette évolution est non linéaire, ce qui montre bien que le premier régime non linéaire est, en général, celui des oscillations fortes.

- La question est ensuite de savoir si les solutions approchées $u_{a}^{\varepsilon}$ construites par la méthode BKW sont effectivement asymptotes à des solutions exactes sur un intervalle de temps $[0, T]$ uniforme en $\varepsilon$. C'est la question de la stabilité des solutions approchées. La discussion porte d'abord sur le linéarisé $\mathcal{L}_{c}$ de (1.1) autour d'une solution approchée $u_{a}^{\varepsilon}$. Ce linéarisé contient des termes singuliers en $\varepsilon^{-1}$ et en $\varepsilon^{-1 / 2}$ qui font que l'hyperbolicité ne suffit pas à assurer la validité d'estimations à priori uniformes en $\varepsilon$.

En dimension $d=1$, la stabilité est obtenue en utilisant seulement l'existence d'un bon symétriseur cf. $[6,13,22]$. En dimension $d>1$, cela ne suffit pas à garantir la stabilité, comme le montre la dynamique des gaz. L'existence d'un bon symétriseur n'empêche pas les perturbations dans des directions transverses à la phase $\varphi$ de donner lieu à des instabilités fortes de type Rayleigh cf. [9,10]. En fait il semble que ce phénomène soit de portée générale.

On organise la discussion de la stabilité en deux parties. Au paragraphe 5, on discute la signification intrinsèque des termes singuliers, ainsi que la mise du linéarisé sous forme canonique. On obtient la stabilité linéaire et non linéaire des solutions BKW sous deux hypothèses. La première est l'existence d'un bon symétriseur. La seconde exige que la valeur propre linéairement dégénérée ne dépende pas de $u$. La première hypothèse est réaliste au vu des applications mais la seconde est très restrictive.

Dans un deuxième temps, au paragraphe 6, on s'intéresse à la cause des instabilités. Les instabilités sont dues à l'interaction des oscillations de phase $\varphi$ avec des oscillations de phase $\psi$ arbitraire. Si l'hypothèse de dégénérescence linéaire de la valeur propre implique que les coefficients d'auto-interaction s'annulent (c'est-à-dire donne accès à certaines conditions de transparence), il n'en est pas de même pour les coefficients d'interaction entre ondes associées à des phases différentes. On est donc amené à faire une analyse de type optique géométrique multi-phases pour le linéarisé $\mathcal{L}_{c}$ et cette optique-là n'a pas de raisons d'être transparente. Par exemple, pour les équations de la dynamique des gaz, l'analyse des oscillations transverses fait apparaître le système linéarisé des équations d'Euler incompressibles, qui donne lieu aux instabilités de Rayleigh (cf. [9]). Dans ce cas, l'équation linéarisée $\mathcal{L}_{c} \dot{u}=0$ admet des solutions qui croissent exponentiellement en temps, en $e^{\gamma t / \sqrt{\varepsilon}}$. Comme dans [10], ces instabilités linéaires exponentielles donnent lieu à des instabilités non linéaires (cf. paragraphe 6; voir aussi [15] dans le cas semi-linéaire). Si l'on n'énonce pas de théorème précis dans ce sens, il semble que les conditions qui conduisent à ces instabilités exponentielles sont générales.

L'analyse ci-dessus ne clôt pas la question de la stabilité des solutions BKW. Dans certains cas, le linéarisé peut être faiblement stable (ou faiblement instable), c'est-à-dire que les solutions de $\mathcal{L}_{c} \dot{u}=0$ sont à croissance au plus polynomiale en $\varepsilon^{-1}$. Il est alors naturel de chercher des estimations uniformes en pondérant avec des puissances de $\varepsilon$ les différentes composantes du vecteur $u$. Par un changement d'inconnues singulier en $\varepsilon$, on peut alors espérer se ramener à des équations linéairement et non linéairement uniformément stables pour les nouvelles inconnues. Cette stratégie est effectivement gagnante en dimension d'espace égale à un (cf. [6,13]) ou, dans le cas des équations d'Euler, pour des oscillations $u_{1}$ portées uniquement sur l'entropie (cf. [4]). L'idée d'utiliser un changement d'inconnues singulier est présentée dans [15] dans le contexte des équations semi-linéaires. 
Enfin, les calculs BKW effectués pour les oscillations fortes, et l'analyse de stabilité ci-dessus, indiquent quelles sont les contraintes qui permettent d'envisager avec efficacité l'existence d'oscillations de grande amplitude. Les conditions requises portent à la fois sur la structure du système, la polarisation du profil principal des oscillations et sur le choix de la fonction $\bar{u}_{0}$. Pour la dynamique des gaz, cela revient à considérer des oscillations dont le terme principal est polarisé sur l'entropie uniquement. La construction sous ces hypothèses et l'analyse de solutions (1.2) avec $n_{0}=0$ est l'objet de [4].

Notre article est organisé comme suit. Le paragraphe 2 précise le contexte (équations, notations, définitions, ...). Le chapitre 3 regroupe les résultats principaux. Le paragraphe 4 se rapporte à la construction des solutions approchées. Les parties 5 et 6 parlent de stabilité et d'instabilité.

\section{Le contexte}

\subsection{Les équations}

On considère le système de lois de conservation (1.1). Les flux $f_{j}$ sont des fonctions $C^{\infty}$ sur l'ouvert $\mathcal{U} \subset \mathbb{R}^{N}$ à valeurs dans $\mathbb{R}^{N}$. On suppose que le système est hyperbolique symétrisable dans la direction du temps :

HYPOTHÈSE 2.1. - Il existe une matrice $\Sigma_{0}(u)$ symétrique définie positive, de classe $C^{\infty}$ sur $\mathcal{U}$ et telle que les d matrices:

$$
\Sigma_{j}(u):=\Sigma_{0}(u) f_{0}^{\prime}(u)^{-1} f_{j}^{\prime}(u), \quad j \in\{1, \ldots, d\}
$$

sont symétriques pour tout $u \in \mathcal{U}$.

L'hypothèse 2.1 est satisfaite par de nombreux exemples physiques et en particulier dès que le système admet une entropie strictement convexe [8]. Sous l'hypothèse 2.1, la matrice

$$
A(u, \xi):=\sum_{j=1}^{d} \xi_{j} f_{0}^{\prime}(u)^{-1} f_{j}^{\prime}(u)
$$

possède des valeurs propres qui sont toutes réelles. On en sélectionne une, notée $\lambda(u, \xi)$. On pose $E(u, \xi):=\operatorname{ker}(A(u, \xi)-\lambda(u, \xi) \mathrm{Id})$.

La plupart des systèmes issus de la physique possèdent une valeur propre linéairement dégénérée (en abrégé 1.d.g.) ce qui motive :

HYPOTHÈSE 2.2. - La valeur propre $\lambda$ est linéairement dégénérée et de multiplicité constante $\kappa$ pour $(u, \xi)$ parcourant $\mathcal{U} \times \mathbb{S}^{d-1}$.

On a noté $\mathbb{S}^{d-1}$ la sphère unité de $\mathbb{R}^{d}$. L'hypothèse 2.2 impose :

$$
\begin{aligned}
& \exists \kappa \in \mathbb{N}^{*}, \forall(u, \xi) \in \mathcal{U} \times \mathbb{S}^{d-1}: \quad \operatorname{dim} E(u, \xi)=\kappa, \\
& \forall(u, \xi) \in \mathcal{U} \times \mathbb{S}^{d-1}, \forall v \in E(u, \xi): \quad v \cdot \nabla_{u} \lambda(u, \xi)=0 .
\end{aligned}
$$

Pour $\kappa>1$, il n'est pas nécessaire d'ajouter (2.3) car la relation (2.3) est alors une conséquence de (2.2) (voir Boillat [2]). Un résultat important est que sous l'hypothèse 2.2, pour tout $\xi \neq 0$, le champ de $\kappa$-plans $E(\cdot, \xi)$ est intégrable (cf. [2,23]). Il engendre donc un feuilletage noté $\Im_{\xi}$. Localement, il existe un changement de variables $\widetilde{u}=(z, w) \in \mathbb{R}^{N-\kappa} \times \mathbb{R}^{\kappa} \mapsto \Phi_{\xi}(\widetilde{u})=u$ tel 
que pour le nouveau système écrit pour l'inconnue $\widetilde{u}$, l'espace propre correspondant $\widetilde{E}(\widetilde{u}, \xi):=$ $\left[D_{\widetilde{u}} \Phi_{\xi}(\widetilde{u})\right]^{-1} E\left(\Phi_{\xi}(u), \xi\right)$ vérifie :

$$
\forall \widetilde{u}, \quad \widetilde{E}(\widetilde{u}, \xi)=\left\{(0, w): w \in \mathbb{R}^{\kappa}\right\}
$$

Dans toute la suite, on suppose que les hypothèses 2.1 et 2.2 sont satisfaites.

\subsection{Les phases et les profils}

On s'intéresse au régime des oscillations fortes. Le développement asymptotique (1.2) s'écrit

$$
\begin{aligned}
& u^{\varepsilon}(t, x) \underset{\varepsilon \rightarrow 0}{\sim} u_{a}^{\varepsilon}\left(t, x, \frac{\varphi_{\varepsilon}}{\varepsilon}\right), \\
& u_{a}^{\varepsilon}(t, x, \theta)=\bar{u}_{0}(t, x)+\sum_{n=1}^{n_{1}}(\sqrt{\varepsilon})^{n} u_{n}(t, x, \theta) .
\end{aligned}
$$

On cherche des solutions telles que $\partial_{\theta} u_{1} \not \equiv 0$, qui échappent donc au cadre faiblement non linéaire traité dans $[11,12,18]$. L'objectif de ce paragraphe est de décrire les ingrédients $\varphi_{\varepsilon}, \bar{u}_{0}$ et $u_{n}$ pour $1 \leqslant n \leqslant n_{1}$.

Le problème étant hyperbolique, on pourrait s'intéresser à des solutions locales en espace et en temps de (1.1). Néanmoins, pour simplifier, on fera des hypothèses globales en espace. Par vitesse finie de propagation, les résultats locaux s'en déduisent.

Les profils. $\quad$ En (2.6), $\bar{u}_{0}(t, x)$ est une solution particulière du système (1.1), définie sur $[0, T] \times \mathbb{R}^{d}$ avec $T>0$ :

$$
\partial_{j} f_{0}\left(\bar{u}_{0}\right)+\sum_{j=1}^{d} \partial_{x_{j}} f_{j}\left(\bar{u}_{0}\right)=0, \quad \operatorname{sur}[0, T] \times \mathbb{R}^{d} .
$$

La fonction $\bar{u}_{0}$ prend ses valeurs dans un compact de l'ouvert $\mathcal{U}$. Elle est dans l'espace $C_{b}^{\infty}(T)$ des fonctions qui sont bornées et dont les dérivées à tout ordre sont bornées sur $[0, T] \times \mathbb{R}^{d}$. Par exemple, on peut choisir $\bar{u}_{0}(t, x)=\underline{u}$ constante. Dans toute la suite, la fonction $\bar{u}_{0}$ est considérée comme une donnée fixée.

Pour $n \geqslant 1, u_{n}(t, x, \theta)$ est une fonction des variables lentes $(t, x) \in[0, T] \times \mathbb{R}^{d}$ et de la variable rapide $\theta \in \mathbb{T}:=\mathbb{R} /(2 \pi \mathbb{Z})$. Elle est périodique de période $2 \pi$ en $\theta$. Elle se sépare en :

$$
u_{n}(t, x, \theta)=\bar{u}_{n}(t, x)+u_{n}^{*}(t, x, \theta) .
$$

On a noté respectivement $\bar{u}(t, x)$ et $u^{*}(t, x, \theta)$ la moyenne et l'oscillation de la fonction $u(t, x, \theta)$, qui est périodique en $\theta$. On a :

$$
\bar{u}(t, x)=\langle u\rangle(t, x):=\frac{1}{2 \pi} \int_{\mathbb{T}} u(t, x, \theta) d \theta, \quad u^{*}=u-\bar{u} .
$$

Les profils sont cherchés dans des espaces de Sobolev basés sur $L^{2}$. Pour $s$ entier, $s>\frac{d+3}{2}$ et $T>0$, on considère l'espace

$$
\mathcal{W}^{s}(T):=\left\{u ; u \in C^{j}\left([0, T] ; H^{s-j}\left(\mathbb{R}^{d} \times \mathbb{T}\right)\right), \forall j \in\{0, \ldots, s\}\right\} .
$$


On note $H^{\infty}$ l'intersection des espaces $H^{s}$ et $\mathcal{W}^{\infty}(T)$ l'intersection des espaces $\mathcal{W}^{s}(T)$. On définit des espaces similaires sur $[0, T] \times \mathbb{R}^{d}$ pour des fonctions indépendantes de $\theta$. Pour simplifier, et sans risque de confusion, on notera encore $\mathcal{W}^{s}(T)$ les espaces correspondants.

La moyenne $\bar{u}_{1}$ du premier profil $u_{1}$ est une solution dans $\mathcal{W}^{\infty}(T)$ de l'équation linéarisée

$$
\begin{aligned}
& f_{0}^{\prime}\left(\bar{u}_{0}\right) \partial_{t} \bar{u}_{1}+\sum_{j=1}^{d} f_{j}^{\prime}\left(\bar{u}_{0}\right) \partial_{x_{j}} \bar{u}_{1}+\left(D_{u}^{2} f_{0}\right)\left(\bar{u}_{0}\right)\left(\bar{u}_{1}, \partial_{t} \bar{u}_{0}\right) \\
& +\sum_{j=1}^{d}\left(D_{u}^{2} f_{j}\right)\left(\bar{u}_{0}\right)\left(\bar{u}_{1}, \partial_{x_{j}} \bar{u}_{0}\right)=0 .
\end{aligned}
$$

Étant donnée une condition initiale dans $H^{\infty}\left(\mathbb{R}^{d}\right)$ l'équation (2.9) détermine $\bar{u}_{1} \in \mathcal{W}^{\infty}(T)$.

Les oscillations $u_{n}^{*}$ et les moyennes $\bar{u}_{n+1}$ sont, pour $n \geqslant 1$, déterminées à l'aide d'équations qui seront décrites au cours du paragraphe 4 .

Les phases. La phase $\varphi_{\varepsilon}(t, x)$ se partage en :

$$
\varphi_{\varepsilon}(t, x):=\varphi(t, x)+\sqrt{\varepsilon} \varphi^{1}(t, x) .
$$

La phase dominante $\varphi(t, x)$ est une fonction $C^{\infty}$ définie sur $[0, T] \times \mathbb{R}^{d}$, à valeurs dans $\mathbb{R}$, solution de l'équation eikonale :

$$
\partial_{t} \varphi+\lambda\left(\bar{u}_{0}, \partial_{x} \varphi\right)=0, \quad \varphi(0, x)=\varphi_{0}(x) .
$$

On suppose que $d \varphi \in C_{b}^{\infty}(T)$ et qu'elle est non stationnaire :

$$
\exists c>0, \forall(t, x) \in[0, T] \times \mathbb{R}^{d}, \quad\left|\partial_{x} \varphi(t, x)\right| \geqslant c .
$$

Par exemple, si $\bar{u}_{0}$ est une constante $\underline{u}$, on peut choisir pour $\varphi$ une phase plane

$$
\varphi(t, x)=\underline{\tau} t+\underline{\xi} \cdot x, \quad \text { avec } \underline{\xi} \neq 0 \text { et } \underline{\tau}=-\lambda(\underline{u}, \underline{\xi}) .
$$

L'expression $\varphi^{1} \in \mathcal{W}^{\infty}(T)$ s'obtient par résolution de :

$$
\left\{\begin{array}{l}
\partial_{t} \varphi^{1}+\left(\partial_{x} \varphi^{1} \cdot \nabla_{\xi}\right) \lambda\left(\bar{u}_{0}, \partial_{x} \varphi\right)+\left(\bar{u}_{1} \cdot \nabla_{u}\right) \lambda\left(\bar{u}_{0}, \partial_{x} \varphi\right)=0 \\
\varphi^{1}(0, x)=0 .
\end{array}\right.
$$

Avec (2.10), le développement asymptotique (1.2) peut s'interpréter selon une optique géométrique bi-phase :

$$
\dot{u}^{\varepsilon}(t, x) \sim \bar{u}_{0}(t, x)+\sum_{n=1}^{+\infty}(\sqrt{\varepsilon})^{n} \tilde{u}_{n}\left(t, x, \frac{\varphi^{1}(t, x)}{\sqrt{\varepsilon}}, \frac{\varphi(t, x)}{\varepsilon}\right)
$$

où les $\tilde{u}_{n}$ se déduisent des $u_{n}$. Notons ici que la phase $\varphi^{1}$ est figée par les choix de $\bar{u}_{0}, \bar{u}_{1}$ et $\varphi$. La fonction $\varphi^{1}$ joue le rôle d'un terme de correction (sur lequel on ne dispose d'aucune liberté). On peut se ramener (voir le paragraphe 4.3) au cas particulier où $\bar{u}_{1}=0$ et $\varphi^{1}=0$. Le développement (1.2) fait alors intervenir une seule phase $\varphi_{\varepsilon}=\varphi$ qui ne dépend plus du paramètre $\varepsilon$. 


\subsection{Notations}

Ce paragraphe regroupe la plupart des notations qui sont utilisées par la suite. Le lecteur qui s'interroge sur la définition d'une expression peut s'y reporter. On écrit en abrégé :

$$
\partial_{0}:=\partial_{t}, \quad \partial_{d+1}:=\partial_{\theta}, \quad \partial_{j}:=\partial_{x_{j}}, \quad 1 \leqslant j \leqslant d .
$$

On travaille avec les variables éclatées $(t, x, \theta)$. On résout :

$$
\mathcal{S}\left(u^{\varepsilon} ; \partial_{t, x, \theta}\right) u^{\varepsilon}=\sum_{j=0}^{d} \Sigma_{j}\left(u^{\varepsilon}\right) \partial_{j} u^{\varepsilon}+\frac{1}{\varepsilon} \sum_{j=0}^{d} \partial_{j} \varphi_{\varepsilon} \Sigma_{j}\left(u^{\varepsilon}\right) \partial_{\theta} u^{\varepsilon}=0
$$

où les matrices $\Sigma_{j}(u)$ sont définies en $(2.1)$. Toute solution $u^{\varepsilon}(t, x, \theta)$ du système $(\mathcal{S})$ fournit une solution $\tilde{u}^{\varepsilon}(t, x)=u^{\varepsilon}\left(t, x, \frac{\varphi_{\varepsilon}}{\varepsilon}\right)$ du système (1.1).

Nous noterons $S(u, \tau, \xi)$ la matrice symétrique

$$
S(u, \tau, \xi):=\tau \Sigma_{0}(u)+\sum_{j=1}^{d} \xi_{j} \Sigma_{j}(u) .
$$

Soit $k$ un entier non nul. On note $\mathcal{L}_{k}\left(\mathbb{R}^{l} ; \mathbb{R}^{m}\right)$ l'ensemble des applications $k$-linéaires symétriques sur $\mathbb{R}^{l}$ à valeurs dans $\mathbb{R}^{m}$. Pour $\xi \in \mathbb{R}^{d}$ et $u \in \mathcal{U}$ on désigne par $L_{k}(u, \xi)$ l'élément de $\mathcal{L}_{k}\left(\mathbb{R}^{N} ; \mathbb{R}^{N}\right)$ qui est défini par la relation :

$$
k ! f_{0}^{\prime}(u) L_{k}(u, \xi):=\sum_{j=1}^{d} \xi_{j}\left(D_{u}^{k} f_{j}\right)(u)-\lambda(u, \xi)\left(D_{u}^{k} f_{0}\right)(u) .
$$

En particulier, $L_{1}(u, \xi)=A(u, \xi)-\lambda(u, \xi) \operatorname{Id} \in \mathcal{L}_{1}\left(\mathbb{R}^{N} ; \mathbb{R}^{N}\right)$. On introduit aussi la forme symétrique

$$
\Sigma(u, \xi):=\Sigma_{0}(u) L_{1}(u, \xi)=-\lambda(u, \xi) \Sigma_{0}(u)+\sum_{j=1}^{d} \xi_{j} \Sigma_{j}(u),
$$

de sorte que $E(u, \xi)=\operatorname{ker} \Sigma(u, \xi)$ On remarque que :

$$
S(u, \tau, \xi)=\Sigma(u, \xi)+(\tau+\lambda(u, \xi)) \Sigma_{0}(u) .
$$

On désigne par $\Pi(u, \xi)$, une application $C^{\infty} \operatorname{sur} \mathcal{U} \times \mathbb{R}^{d} \backslash\{0\}$ telle que pour tout $(u, \xi), \Pi(u, \xi)$ est un projecteur de $\mathbb{R}^{N}$ sur $\operatorname{ker} L_{1}(u, \xi)=\operatorname{ker} \Sigma(u, \xi)$ :

$$
L_{1}(u, \xi) h=0 \quad \Leftrightarrow \quad h=\Pi(u, \xi) h .
$$

En particulier, $L_{1}(u, \xi) \Pi(u, \xi)=0$. On se donne une application $Q \in C^{\infty}\left(\mathcal{U} \times \mathbb{R}^{d} \backslash\{0\} ; \mathcal{L}\left(\mathbb{R}^{N}\right)\right)$ telle que $Q(u, \xi)$ soit un inverse partiel de $L_{1}(u, \xi)$ à valeurs dans $\operatorname{ker} \Pi(u, \xi)$ :

$$
Q(u, \xi) L_{1}(u, \xi)=\mathrm{Id}-\Pi(u, \xi), \quad \Pi(u, \xi) Q(u, \xi)=0 .
$$

Enfin on se donne une application $\Pi^{\prime}(u, \xi), C^{\infty} \operatorname{sur} \mathcal{U} \times \mathbb{R}^{d} \backslash\{0\}$ telle que

$$
\operatorname{ker} \Pi^{\prime}(u, \xi)=\operatorname{im} L_{1}(u, \xi) \text {. }
$$


En particulier, $\Pi^{\prime}(u, \xi) L_{1}(u, \xi)=0$. D'autre part, comme $\lambda$ est une valeur propre semi-simple de $A(u, \xi)$, en tout point $(u, \xi) \in \mathcal{U} \times \mathbb{R}^{d} \backslash\{0\}$ on a im $L_{1} \cap \operatorname{ker} L_{1}=\{0\}$ et

$$
\operatorname{rang}\left(\Pi^{\prime} \Pi\right)=\operatorname{rang} \Pi^{\prime}=\operatorname{rang} \Pi=\kappa .
$$

Les identités ci-dessus impliquent que pour tout $(u, \xi) \in \mathcal{U} \times \mathbb{R}^{d} \backslash\{0\}$ :

$$
f=0 \Leftrightarrow\left\{\begin{array}{l}
\Pi^{\prime}(u, \xi) f=0, \\
Q(u, \xi) f=0 .
\end{array}\right.
$$

Exemples. Plusieurs choix sont naturels. On peut choisir $\Pi=\Pi^{\prime}=\Pi_{S}$ le projecteur spectral de $A(u, \xi)$ associé à la valeur propre $\lambda(u, \xi)$. On peut aussi choisir $\Pi=\Pi_{\perp}$ le projecteur orthogonal sur ker $L_{1}=\operatorname{ker} \Sigma$. Par symétrie on a aussi $\Pi_{\perp} \Sigma=0$ et on peut par exemple choisir $\Pi^{\prime}=\Sigma_{0}^{-1} \Pi_{\perp} \Sigma_{0}$. Dans la pratique, il est parfois intéressant de faire d'autres choix. Par exemple, $\Pi^{\prime}$ peut être donné par une base du co-noyau de $L_{1}$ (vecteurs propres à gauche). Quant à l'inverse partiel, il est uniquement déterminé de im $L_{1}$ dans ker $\Pi$. Sa définition sur un supplémentaire de $\operatorname{im} L_{1}$ n'intervient pas.

On définit un élément $\Gamma_{2}(u, \xi)$ dans $\mathcal{L}_{2}\left(\mathbb{R}^{N} ; \mathbb{R}^{N}\right)$ par :

$$
\Gamma_{2}(u, \xi)\left(w_{1}, w_{2}\right):=-2 Q(u, \xi) L_{2}(u, \xi)\left(w_{1}, w_{2}\right) .
$$

Enfin, on introduit les formes trilinéaires :

$$
\begin{aligned}
& 2 B l^{\prime}(u, \xi)\left(u_{1} ; v, w\right):=-\left(\left(u_{1} \cdot \nabla_{u} \Sigma\right) v, w\right)+\left(\left(v \cdot \nabla_{u} \Sigma\right) u_{1}, w\right)+\left(\left(w \cdot \nabla_{u} \Sigma\right) u_{1}, v\right) \\
& 2 B l^{\prime \prime}(u, \xi)\left(u_{1} ; v, w\right):=\left(\left(v \cdot \nabla_{u} \lambda\right) \Sigma_{0} u_{1}, w\right)+\left(\left(w \cdot \nabla_{u} \lambda\right) \Sigma_{0} u_{1}, v\right)
\end{aligned}
$$

Ici les applications $\nabla_{u} \Sigma, \nabla_{u} \lambda$ et $\Sigma_{0}$ sont évaluées en $(u, \xi)$. On remarque que ces formes sont symétriques en $(v, w)$. On note $B l=B l^{\prime}+B l^{\prime \prime}$.

Une fonction qui dépend des variables $(u, \xi)$ donne lieu, après substitution de $(u, \xi)$ par $\left(\bar{u}_{0}, \partial_{x} \varphi\right)$, à une fonction de $(t, x)$. On définit ainsi :

$$
\begin{aligned}
& v \cdot \nabla \lambda:=v \cdot \nabla_{u} \lambda\left(\bar{u}_{0}, \partial_{x} \varphi\right), \quad \mathcal{L}_{k}:=L_{k}\left(\bar{u}_{0}, \partial_{x} \varphi\right), \\
& \mathcal{P}:=\Pi\left(\bar{u}_{0}, \partial_{x} \varphi\right), \quad \mathcal{P}^{\prime}:=\Pi^{\prime}\left(\bar{u}_{0}, \partial_{x} \varphi\right), \quad \mathcal{Q}:=Q\left(\bar{u}_{0}, \partial_{x} \varphi\right), \\
& \mathcal{B} l^{\prime}=B l^{\prime}\left(\bar{u}_{0}, \partial_{x} \varphi\right), \quad \mathcal{B} l^{\prime \prime}=B l^{\prime \prime}\left(\bar{u}_{0}, \partial_{x} \varphi\right), \quad \mathcal{B} l=B l\left(\bar{u}_{0}, \partial_{x} \varphi\right) .
\end{aligned}
$$

Par souci de simplification, on ne fait pas mention dans ces expressions des variables $(t, x)$. Cela ne doit pas prêter à confusion. Les relations précédentes se transcrivent comme suit :

$$
\begin{aligned}
& \mathcal{L}_{1} \mathcal{P}=0 \quad \text { et } \quad \operatorname{ker} \mathcal{L}_{1}=\operatorname{im} \mathcal{P}, \\
& \mathcal{Q} \mathcal{L}_{1}=\operatorname{Id}-\mathcal{P}, \quad \mathcal{Q P}=\mathcal{P} \mathcal{Q}=0 \\
& \mathcal{P}^{\prime} \mathcal{L}_{1}=0 \quad \text { et } \quad \operatorname{im} \mathcal{L}_{1}=\operatorname{ker} \mathcal{P}^{\prime}
\end{aligned}
$$

On a aussi, en tout point $(t, x)$ :

$$
f=0 \Leftrightarrow\left\{\begin{array}{l}
\mathcal{P}^{\prime} f=0 \\
\mathcal{Q} f=0
\end{array}\right.
$$

\subsection{Définitions}

Ce paragraphe est destiné à donner un sens précis aux diverses terminologies qui ont été employées. 
Solutions approchées. On cherche à résoudre l'équation $(\mathcal{S})$ dans les espaces $\mathcal{W}^{s}(T)$ avec $s$ assez grand. On munit ces espaces de la famille de normes :

$$
\left.\left.\|u\|_{\mathcal{W}_{\varepsilon}^{s}(T)}:=\sup _{t \in[0, T]} \sum_{|\alpha| \leqslant s}\left\|\varepsilon^{|\alpha|} \partial_{t, x, \theta}^{\alpha} u(t, \cdot)\right\|_{L^{2}\left(\mathbb{R}^{d} \times \mathbb{T}\right)}, \quad \varepsilon \in\right] 0,1\right] .
$$

Pour $\varepsilon=1$, on note simplement $\|\cdot\|_{\mathcal{W}^{s}(T)}$. Pour $\left.\left.\varepsilon \in\right] 0,1\right]$, on a clairement :

$$
\varepsilon^{s}\|u\|_{\mathcal{W}^{s}(T)} \leqslant\|u\|_{\mathcal{W}_{\varepsilon}^{s}(T)} \leqslant\|u\|_{\mathcal{W}^{s}(T)}
$$

On dit que la famille $\left\{u^{\varepsilon}\right\}_{\varepsilon \in] 0,1]}$ est bornée dans $\mathcal{W}_{\varepsilon}^{s}(T)$ si on a la majoration uniforme : $\sup _{\varepsilon \in] 0,1]}\left\|u^{\varepsilon}\right\|_{\mathcal{W}_{\varepsilon}^{s}(T)}<+\infty$.

Par extension, $\mathcal{W}^{s}(+\infty)$ est l'espace des fonctions $u(t, \cdot)$ définies sur $\mathbb{R}^{+}$et telles que $u$ soit dans $\mathcal{W}^{s}(T)$ pour tout $T<+\infty$. De même, on dit que la famille $\left\{u^{\varepsilon}\right\}_{\varepsilon \in] 0,1]}$ est bornée dans $\mathcal{W}_{\varepsilon}^{s}(+\infty)$ si elle est bornée dans $\mathcal{W}_{\varepsilon}^{s}(T)$ pour tout $T<+\infty$.

On considère des profils $u_{n}$ choisis dans $\mathcal{W}^{s+1}(T)$. Pour $n_{1}<+\infty$, par la formule (2.6) on définit $u_{a}^{\varepsilon}-\bar{u}_{0}=\sum_{n=1}^{n_{1}} \varepsilon^{n / 2} u_{n}$ comme une famille bornée dans $\mathcal{W}^{s+1}(T)$. Pour $n_{1}=+\infty$, par le procédé de sommation de Borel on définit une famille $u_{a}^{\varepsilon}$ bornée dans $\mathcal{W}^{s+1}(T)$ et telle que

$$
u_{a}^{\varepsilon}-\bar{u}_{0} \sim \sum_{n=1}^{\infty} \varepsilon^{n / 2} u_{n}
$$

Cette famille $u_{a}^{\varepsilon}$ est définie modulo une famille qui est $\mathrm{O}\left(\varepsilon^{\infty}\right)$ dans $\mathcal{W}^{s+1}(T)$.

Si $s>\frac{d+3}{2}$, pour tout $\left.\left.\varepsilon \in\right] 0,1\right]$ la fonction $u_{a}^{\varepsilon}$ est de classe $C^{1}$ et on a :

$$
R_{a}^{\varepsilon}:=\mathcal{S}\left(u_{a}^{\varepsilon} ; \partial_{t, x, \theta}\right) u_{a}^{\varepsilon} \in \mathcal{W}^{s}(T)
$$

C'est encore vrai pour $s=\infty$.

DÉFINITION 2.1. - Soit $r$ avec $0 \leqslant r \leqslant+\infty$. On dit que la famille $\left\{u_{a}^{\varepsilon}\right\}_{\varepsilon \in] 0,1]}$, donnée par (2.6) ou (2.32) si $n_{1}=\infty$, avec les $u_{n} \in \mathcal{W}^{s+1}(T)$, est une solution approchée du système $(\mathcal{S})$ à l'ordre $r$ dans $\mathcal{W}^{s}(T)$ si :

$$
\left\|R_{a}^{\varepsilon}\right\|_{\mathcal{W}^{s}(T)}=\mathrm{O}\left(\varepsilon^{r}\right) \text {. }
$$

On note $\mathcal{O}_{a}^{s}(r)$ l'ensemble des solutions approchées d'ordre $r$ à coefficients $u_{n} \in \mathcal{W}^{s+1}(T)$.

Stabilité. Une fois construites les solutions approchées, la question est de savoir si elles sont proches de solutions exactes. On formalise ici cette notion de stabilité. On se donne $r>s+1>\frac{d+5}{2}$.

DÉfINITION 2.2. - On dit que la famille de solutions approchées $\left\{u_{a}^{\varepsilon}\right\}_{\varepsilon \in] 0,1]} \in \mathcal{O}_{a}^{s}(r)$ est stable sur $[0, T]$ si la condition suivante est satisfaite. Pour toute famille $\left\{R^{\varepsilon}\right\}_{\varepsilon \in] 0,1]}$ bornée dans $\mathcal{W}_{\varepsilon}^{s}(T)$ et toute famille $\left\{I^{\varepsilon}\right\}_{\varepsilon \in] 0,1]}$ bornée dans $H^{s}\left(\mathbb{R}^{d} \times \mathbb{T}\right)$, il existe un seuil $\varepsilon_{0}>0$ tel que pour tout $\left.\varepsilon \in] 0, \varepsilon_{0}\right]$, le problème de Cauchy :

$$
\mathcal{S}\left(u^{\varepsilon} ; \partial_{t, x, \theta}\right) u^{\varepsilon}=R_{a}^{\varepsilon}+\varepsilon^{r} R^{\varepsilon}, \quad u^{\varepsilon}(0, x, \theta)=u_{a}^{\varepsilon}(0, x, \theta)+\varepsilon^{r} I^{\varepsilon}
$$

admet une solution unique $u^{\varepsilon}$ définie sur l'intervalle $[0, T]$ qui vérifie :

$$
\left\|u^{\varepsilon}-u_{a}^{\varepsilon}\right\|_{\mathcal{W}_{\varepsilon}^{s}(T)}=\mathrm{O}\left(\varepsilon^{r}\right) .
$$


Par extension, on dit que la famille $\left\{u_{a}^{\varepsilon}\right\}_{\varepsilon \in] 0,1]}$ est stable sur $[0,+\infty]$ si elle est stable sur tous les intervalles $[0, T]$ avec $0 \leqslant T<+\infty$.

En particulier, en choisissant $R^{\varepsilon}=-\varepsilon^{-r} R_{a}^{\varepsilon}$ et, par exemple, $I^{\varepsilon}=0$, on obtient des solutions exactes $u^{\varepsilon}$ du système $(\mathcal{S})$, définies sur l'intervalle $[0, T]$ et voisine à $\mathrm{O}\left(\varepsilon^{r}\right)$ près de $u_{a}^{\varepsilon}$ au sens de la norme $\|\cdot\|_{\mathcal{W}_{\varepsilon}^{s}(T)}$. Comme $r>s+1>\frac{d+5}{2}$, l'encadrement (2.31), l'injection de Sobolev et l'estimation (2.33) donnent :

$$
\left\|u^{\varepsilon}-u_{a}^{\varepsilon}\right\|_{L^{\infty}\left([0, T] ; \operatorname{Lip}\left(\mathbb{R}^{d} \times \mathbb{T}\right)\right)}=\mathrm{O}(\varepsilon) .
$$

La substitution $\theta=\varphi_{\varepsilon} / \varepsilon$ fournit des solutions $\widetilde{u}^{\varepsilon}(t, x)=u^{\varepsilon}\left(t, x, \varphi_{\varepsilon} / \varepsilon\right)$ du système initial (1.1). On a donc

$$
\left\|\widetilde{u}^{\varepsilon}-\widetilde{u}_{a}^{\varepsilon}\right\|_{L^{\infty}\left([0, T] \times \mathbb{R}^{d}\right)}=\mathrm{O}(\varepsilon),
$$

et aussi :

$$
\left\|\widetilde{u}^{\varepsilon}-\widetilde{u}_{a}^{\varepsilon}\right\|_{L^{\infty}\left([0, T] ; \operatorname{Lip}\left(\mathbb{R}^{d}\right)\right)}=\mathrm{O}(1) .
$$

Lorsque $\partial_{\theta} u_{1} \not \equiv 0$, on observe que la famille $\left\{\widetilde{u}_{a}^{\varepsilon}\right\}_{\varepsilon \in] 0,1]}$ n'est pas bornée dans $W^{1, \infty}$. Il s'ensuit que la famille $\left\{\widetilde{u}^{\varepsilon}\right\}_{\varepsilon \in] 0,1]}$ n'est pas bornée dans $W^{1, \infty}$, ni même à variations locales bornées. En fait les dérivées premières dans les directions transverses à la phase $\varphi$ des solutions $\widetilde{u}^{\varepsilon}$ sont de taille $\frac{1}{\sqrt{\varepsilon}}$.

Lorsque $\partial_{\theta} u_{1} \equiv 0$, le régime est celui de l'optique géométrique faiblement non linéaire. Des résultats standards (cf. [11,12]) affirment que les solutions approchées sont stables au sens de la définition 2.2.

Instabilités non linéaires. Nous décrirons aussi des exemples d'instabilités fortes, du type des instabilités de Rayleigh. Le mécanisme est celui d'une amplification exponentielle en temps rapide $t / \sqrt{\varepsilon}$ des petites perturbations. On formalise ici cette notion d'instabilité (cf. $[10,15]$ ).

DÉFINITION 2.3. - On dit que la famille de solutions approchées $\left\{u_{a}^{\varepsilon}\right\}_{\varepsilon \in] 0,1]} \in \mathcal{O}_{a}^{s}(\infty)$ est non linéairement instable sur $[0, T]$ si la condition suivante est satisfaite. Pour tous $m \in \mathbb{N}$ et $\delta>0$, il existe des constantes $C, \varepsilon_{0}>0$ et $c>0$, et une famille de solutions $\left\{u^{\varepsilon}\right\}_{\varepsilon \in] 0,1]}$ de $(\mathcal{S})$, définies et régulières sur les intervalles $[0, T(\varepsilon)]$, telles que pour tout $\left.\varepsilon \in] 0, \varepsilon_{0}\right]$ on a :

$$
\begin{gathered}
\left\|u_{a}^{\varepsilon}(0)-u^{\varepsilon}(0)\right\|_{L^{\infty}} \leqslant C \varepsilon^{m}, \\
\left\|u_{a}^{\varepsilon}(t)-u^{\varepsilon}(t)\right\|_{L^{\infty}} \geqslant c \sqrt{\varepsilon} .
\end{gathered}
$$

Autrement dit, pour des données initiales $u^{\varepsilon}(0)$ proches à $\varepsilon^{m}$ de $u_{a}^{\varepsilon}(0)$, l'écart entre les solutions exactes $u^{\varepsilon}$ et la solution approchée $u_{a}^{\varepsilon}$ devient, en un temps arbitrairement petit, d'ordre $\sqrt{\varepsilon}$. La taille $\sqrt{\varepsilon}$ correspond à l'amplitude du second terme $\sqrt{\varepsilon} u_{1}$ du développement BKW. Audessus de $\sqrt{\varepsilon}$, le problème étant non linéaire, on ne sait plus estimer la différence qui sépare $u^{\varepsilon}$ et $u_{a}^{\varepsilon}$. C'est pourquoi on s'arrête à l'ordre $\sqrt{\varepsilon}$ et non $\varepsilon^{0}$ en (2.35).

La substitution $\theta=\varphi_{\varepsilon} / \varepsilon$ conduit à des énoncés analogues pour des solutions de l'équation initiale (1.1).

\subsection{Un modèle : la dynamique des gaz}

Les solutions régulières des équations de la dynamique des gaz en dimension $d$ d'espace vérifient le système conservatif :

$$
\left\{\begin{array}{l}
\partial_{t} \varrho+\nabla_{x} \cdot(\varrho v)=0 \\
\partial_{t}\left(\varrho v_{i}\right)+\nabla_{x} \cdot\left(\varrho v v_{i}\right)+\partial_{i} p=0, \quad 1 \leqslant i \leqslant d, \\
\partial_{t}(\varrho s)+\nabla_{x} \cdot(\varrho s v)=0 .
\end{array}\right.
$$


On choisit ici pour variables d'état $(\varrho, v, s, p)$ dans $\mathbb{R}^{+} \times \mathbb{R}^{d} \times \mathbb{R} \times \mathbb{R}^{+}$. Elles représentent respectivement la densité de particules par unité de volume, la vitesse de la particule, la densité d'entropie par unité de masse et de volume, et la pression. Les quantités $\varrho, p$ et $s$ sont liées par une loi d'état $\varrho=\beta(p, s)$ qui vérifie :

$$
\beta(p, s)>0, \quad \frac{\partial \beta}{\partial p}(p, s)>0, \quad p>0, \quad s \in \mathbb{R} .
$$

On manipule le système (2.36) sous une forme quasi-linéaire symétrique, en utilisant les variables $p, v$ et $s$ :

$$
\left\{\begin{array}{l}
\alpha(p, s)\left(\partial_{t} p+v \cdot \nabla_{x} p\right)+\nabla_{x} \cdot v=0 \\
\beta(p, s)\left(\partial_{t} v+v \cdot \nabla_{x} v\right)+\nabla_{x} p=0 \\
\partial_{t} s+v \cdot \nabla_{x} s=0
\end{array}\right.
$$

avec $\alpha(p, s):=\frac{1}{\beta(p, s)} \frac{\partial \beta}{\partial p}(p, s)$. Pour un gaz parfait, $\beta(p, s)=p^{1 / \gamma} e^{-s / \gamma}$ et $\alpha(p, s)=\gamma p$, la constante $\gamma$ étant positive.

Le système (2.36) s'inscrit tout à fait dans le cadre de notre étude. La symétrie (hypothèse 2.1) est évidente sur l'écriture (2.37). La valeur propre $\lambda(u, \xi)=v \cdot \xi$ est linéairement dégénérée et de multiplicité $d$ constante. L'hypothèse 2.2 est donc satisfaite. La discussion met en jeu :

- les lois d'état $\alpha$ et $\beta$.

- le propagateur $\partial_{t}+v \cdot \nabla_{x}$. Cette dérivée particulaire devient en variables éclatées

$$
X:=\partial_{t}+v \cdot \nabla_{x}+\frac{1}{\varepsilon}\left(\dot{X} \varphi_{\varepsilon}\right) \partial_{\theta}
$$

- les matrices :

$$
\Sigma_{0}(u)=\left(\begin{array}{ccc}
\alpha & 0 & 0 \\
0 & \beta \times \operatorname{Id} & 0 \\
0 & 0 & 1
\end{array}\right), \quad \Sigma(u, \xi)=\left(\begin{array}{ccc}
0 & { }^{t} \xi & 0 \\
\xi & 0 & 0 \\
0 & 0 & 0
\end{array}\right) .
$$

$\Sigma_{0}$ est le coefficient de $\partial_{t}$ dans la forme symétrique et $\Sigma$ est défini en (2.17). On remarque que le symbole $\Sigma(u, \xi)=\Sigma(\xi)$ ne dépend pas de l'état $u$. On le note $\Sigma(\xi)=\sum \xi_{j} M_{j}$. Par suite, le projecteur orthogonal $\Pi_{\perp}(u, \xi)=\Pi_{\perp}(\xi)$ sur $\operatorname{ker} \Sigma(\xi)$ ne dépend pas de l'état $u$. Le système éclaté s'écrit alors :

$$
\mathcal{D}\left(u^{\varepsilon} ; \partial_{t, x, \theta}\right) u^{\varepsilon}=\Sigma_{0}\left(u^{\varepsilon}\right) X u^{\varepsilon}+\sum_{j=1}^{d} M_{j} \partial_{j} u^{\varepsilon}+\frac{1}{\varepsilon} \Sigma\left(\partial_{x} \varphi_{\varepsilon}\right) u^{\varepsilon}=0 .
$$

Le système isentropique concerne les solutions pour lesquelles la fonction $s$ reste constante. Il concerne les $d+1$ inconnues $(\varrho, v)$. On récupère alors $\kappa=d-1>0$ si $d>1$.

\section{3. Énoncé des résultats}

\subsection{Solutions BKW}

Le premier résultat important de ce travail concerne l'existence de solutions fortes asymptotiques. On suppose que le système (1.1) vérifie les hypothèses 2.1 et 2.2. On se donne $\bar{u}_{0} \in C_{b}^{\infty}(T)$ solution de (1.1), $\bar{u}_{1} \in \mathcal{W}^{\infty}(T)$ solution de (2.9) et les phases $\varphi$ et $\varphi^{1}$ solutions de (2.11) et (2.14) avec $d \varphi \in C_{b}^{\infty}(T)$ et $\varphi_{1} \in \mathcal{W}^{\infty}(T)$. 
THÉORÈme 3.1. - Il existe des suites de profils $u_{1}^{*}$ et $u_{n}$ pour $2 \leqslant n \leqslant n_{1}$, dans $W^{\infty}(T)$, telles que les fonctions définies par (2.6) sont solutions approchées du système $(\mathcal{S})$ à l'ordre $\left(n_{1}-1\right) / 2$ dans $\mathcal{W}^{s}(T)$ pour tout $s \in \mathbb{N}$. En outre, on peut choisir arbitrairement dans $H^{\infty}\left(\mathbb{R}^{d} \times \mathbb{T}\right)$ les données initiales associées aux composantes $\mathcal{P} u_{n}^{*}$ et $\bar{u}_{n+1}$ pour $n \geqslant 1$.

On obtient aussi des solutions approchées à l'ordre infini en remplaçant la somme de (2.6) par une série asymptotique sommée par le procédé de Borel.

Dans cet énoncé, on a $\mathcal{P}=\Pi\left(\bar{u}_{0}, \partial_{x} \varphi\right)$ comme indiqué au paragraphe 2.3. Le théorème 3.1 est démontré au paragraphe 4. On fait d'abord la démonstration dans le cas où $\bar{u}_{1}=0$ et $\varphi^{1}=0$. En substituant le développement (2.6) dans $\mathcal{S}$ et en ordonnant en puissances de $\varepsilon^{1 / 2}$, on obtient une suite d'équations $F_{n}=0$. La première étape consiste à montrer que ce système équivaut à un système triangulaire de la forme

$$
\left\{\begin{array}{l}
(\mathrm{Id}-\mathcal{P}) u_{n}^{*}=h_{n} \\
\mathcal{Z}_{n}\left(\mathcal{P} u_{n}^{*}, \bar{u}_{n+1}\right)=g_{n}
\end{array}\right.
$$

où $h_{n}$ et $g_{n}$ s'expriment à l'aide des $u_{k}^{*}$ et $\bar{u}_{k+1}$ pour $k \leqslant n-1$ et où $\mathcal{Z}_{n}$ est un système couplé d'équations en $\mathcal{P} u_{n}^{*}$ et $\underline{u}_{n+1}$. Cette réduction des équations $F_{n}=0$ n'est pas du tout évidente. Elle n'est possible que parce que, à chaque étape, un certain nombre de termes s'annulent, comme conséquence des hypothèses de transparence, ici de l'hypothèse 2.2.

La deuxième étape consiste à résoudre les équations $\mathcal{Z}_{n}\left(\mathcal{P} u_{n}^{*}, \bar{u}_{n+1}\right)=g_{n}$. Ceci n'est pas immédiatement clair non plus, le caractère hyperbolique des équations n'étant pas assuré. Néanmoins, en introduisant une inconnue supplémentaire qui s'interprète comme un terme de déphasage $\psi_{n}$ pour $\mathcal{P} u_{n}^{*}$, on résout ce système en déterminant d'abord $v_{n}^{*}=\mathcal{P} u_{n}^{*}\left(t, x, \theta-\psi_{n}\right)$ et $\bar{u}_{n+1}$ comme solution d'un système hyperbolique, puis en résolvant une équation pour $\psi_{n}$.

Enfin, au paragraphe 4.3, par un argument assez simple, on réduit le cas général où $\bar{u}_{1} \neq 0$ au cas précédent.

En particulier, les premiers profils $u_{1}^{*}$ et $\bar{u}_{2}$ sont déterminés par

$$
\left\{\begin{array}{l}
(\operatorname{Id}-\mathcal{P}) u_{1}^{*}=0 \\
\mathcal{Z}_{1}\left(\mathcal{P} u_{1}^{*}, \bar{u}_{2}\right)=0
\end{array}\right.
$$

On reconnait en premier rang la condition de polarisation sur les modes propres associés à la valeur propre $\lambda$. Par contre, on peut choisir la donnée initiale de $\mathcal{P} u_{1}^{*}$ arbitrairement, donc non nulle, créant ainsi de véritables oscillations fortes. Le système $\mathcal{Z}_{1}$ est non linéaire. Néanmoins, la solution existe sur l'intervalle de temps $[0, T]$ en entier. Cela est dû à la réduction triangulaire de ce système qui se ramène à une succession de problèmes linéaires, les non linéarités n'intervenant que comme termes sources identifiés durant les étapes précédentes. Mais les effets non linéaires (interaction entre les différentes composantes, effets des oscillations sur le champ moyen) sont bien présents et décrits par l'équation $\mathcal{Z}_{1}=0$. Ceci indique clairement que le régime pertinent en présence d'un champ l.d.g. est (en général) fourni par les oscillations fortes.

La marge de liberté obtenue sur les données initiales est celle qu'on attend en pareilles circonstances (comparer avec [11] et [12]). Le fait que les données initiales des composantes $(\mathrm{Id}-\mathcal{P}) u_{n}^{*}$ sont déterminées traduit les conditions de compatibilités nécessaires sur les données de Cauchy pour que la solution soit de type (2.6) avec des oscillations sur le seul mode $\lambda$.

L'ordre des solutions approchées peut être fixé quelconque. Il est possible de travailler avec une régularité Sobolev finie. Toutefois, il faut faire un décompte précis des pertes de régularité dans les itérations. Pour récupérer des profils $u_{n}$ dans $\mathcal{W}^{s}$ avec $\frac{d+3}{2}<s<+\infty$, il faut prendre les données initiales dans $H^{s+2 n_{1}}$. Les détails sont laissés au lecteur. 


\subsection{Stabilité hyperbolique}

Il est bien connu que les ondes associées à des modes linéairement dégénérés peuvent donner lieu à des instabilités (cf. [1] pour les discontinuités de contact multidimensionnelles, [9] pour les ondes de cisaillement, [22] pour les oscillations de grande amplitude). Nous allons reprendre cette problématique sous l'angle de l'optique géométrique.

On se donne une solution approchée $u_{a}^{\varepsilon}$ avec $\left\langle u_{1}\right\rangle=0$ et $\varphi_{1}=0$. On analyse d'abord la stabilité linéaire de cette famille. Le linéarisé complet $\mathcal{L}_{c}^{\varepsilon}$ de l'équation $(\mathcal{S})$ en $u_{a}^{\varepsilon}$ est de la forme

$$
\mathcal{L}_{c}^{\varepsilon}=R^{\varepsilon}+\frac{1}{\varepsilon} A_{0} \partial_{\theta}+\frac{1}{\sqrt{\varepsilon}}\left(A_{1} \partial_{\theta}+B_{1}\right)+C^{\varepsilon},
$$

où $R^{\varepsilon}$ est une famille de systèmes symétriques en $\partial_{t, x, \theta}$ à coefficients uniformément Lipschitziens et les $C^{\varepsilon}$ sont uniformément bornés. En outre, $A_{0}=\Sigma\left(\bar{u}_{0}, \partial_{x} \varphi\right)$ est symétrique et indépendant de la variable $\theta$. Par contre, $A_{1}$ est symétrique et dépend de la variable $\theta$ par l'intermédiaire du profil $u_{1}$. De même, $B_{1}$ dépend de $u_{1}$ donc de $\theta$. La méthode d'énergie standard par intégration par parties fait apparaître le terme singulier :

$$
\frac{1}{\sqrt{\varepsilon}}(D \dot{u}, \dot{u}) \quad \text { avec } D:=\frac{1}{2}\left(-\partial_{\theta} A_{1}+B_{1}+{ }^{t} B_{1}\right) .
$$

Avec les notations (2.25) et (2.26) on a :

$$
(D \dot{u}, \dot{u})=B l\left(\bar{u}_{0}, \partial_{x} \varphi\right)\left(\partial_{\theta} u_{1} ; \dot{u}, \dot{u}\right) .
$$

En conséquence, le symétriseur $\Sigma_{0}$, qui conduit à la forme symétrique (3.1), fournit une estimation d'énergie uniforme en $\varepsilon$ pour le linéarisé $\mathcal{L}_{c}^{\varepsilon}$, si et seulement si la matrice symétrique $D$ est positive ou nulle. Comme par construction $D$ est de moyenne nulle, cela impose $D \equiv 0$. La condition $D \equiv 0$ implique la stabilité $L^{2}$ uniforme du linéarisé. On montre qu'elle entraîne aussi la stabilité non linéaire des solutions approchées :

THÉORÈME 3.2. - Soit $u_{a}^{\varepsilon}$ une solution approchée dans $\mathcal{O}_{a}^{s}(r)$ avec $r>s+1>\frac{d+5}{2}$, avec $\bar{u}_{1}=0$. On suppose que les termes $\left(u_{0}, u_{1}^{*}\right)$ et $\varphi$ sont tels que la matrice $D$ définie ci-dessus s'annule sur $[0, T] \times \mathbb{R}^{d} \times \mathbb{T}$. Alors la famille $\left\{u_{a}^{\varepsilon}\right\}_{\varepsilon \in] 0,1]}$ est stable sur l'intervalle $[0, T]$ au sens de la définition 2.2 .

Si $u_{1}^{*} \equiv 0$, alors $D=0$ et le théorème redonne la stabilité de l'optique géométrique faiblement non linéaire [11,12].

On discute maintenant la validité de l'hypothèse $D \equiv 0$ lorsque $u_{1}^{*} \not \equiv 0$. Suivant la décomposition (2.25)-(2.26), on écrit $D=D^{\prime}+D^{\prime \prime}$ avec :

$$
\left(D^{\prime} \dot{u}, \dot{u}\right)=\mathcal{B} l^{\prime}\left(\partial_{\theta} u_{1} ; \dot{u}, \dot{u}\right), \quad\left(D^{\prime \prime} \dot{u}, \dot{u}\right)=\mathcal{B} l^{\prime \prime}\left(\partial_{\theta} u_{1} ; \dot{u}, \dot{u}\right) .
$$

On lie d'abord la condition $D^{\prime}=0$ à la notion de bon symétriseur introduite dans $[6,13,24]$. Cette notion est une notion $1 \mathrm{D}$, qui s'applique dans chaque direction $\xi \in \mathbb{S}^{d-1}$ au système :

$$
\partial_{t} f_{0}(u)+\partial_{x} f_{\xi}(u)=0, \quad f_{\xi}(u):=\sum_{j=1}^{d} \xi_{j} f_{j}(u) .
$$

La proposition suivante est démontrée au paragraphe 4. 
PROPOSITION 3.1. - Les conditions suivantes sont équivalentes.

(i) Pour toute direction $\xi$ dans $\mathbb{S}^{d-1}$, la matrice $\Sigma_{0}(u)$ est un bon symétriseur au sens donné dans $[6,13,24]$ pour le système $(3.5)$.

(ii) Pour toute direction $\xi$ dans $\mathbb{S}^{d-1}$, la dérivée de Lie du tenseur 2 fois covariant $\Sigma(u, \xi)$ le long du feuilletage $\Im_{\xi}$ est nulle.

(iii) Pour tout $(u, \xi) \in \mathcal{U} \times \mathbb{S}^{d-1}$ et tout $u_{1} \in E(u, \xi)$, la forme $B l^{\prime}(u, \xi)\left(u_{1} ; \cdot, \cdot\right)$ est nulle.

Lorsque ces conditions sont satisfaites, on dira que $\Sigma_{0}$ est un bon symétriseur pour l'équation (1.1). La définition de bon symétriseur 1D est rappelée au paragraphe 4. Elle s'exprime simplement dans des variables où le champ $E(u, \xi)$ est redressé comme en (2.4), et (ii) en donne la définition intrinsèque invariante par changement d'inconnues. L'existence d'un bon symétriseur $\Sigma_{0}$ est un point clé dans la preuve de la stabilité 1D (cf. [13,6]). Cette condition est satisfaite dans de nombreux cas, en particulier par le système d'Euler $(\mathcal{D})$. On retient que si $\Sigma_{0}$ est un bon symétriseur, alors le terme singulier $\left(D^{\prime} \dot{u}, \dot{u}\right)$ s'annule.

En ce qui concerne le terme $\left(D^{\prime \prime} \dot{u}, \dot{u}\right)$, on a :

$$
\left(D^{\prime \prime} \dot{u}, \dot{u}\right)=(\dot{u} \cdot \nabla \lambda)\left(\Sigma_{0} \partial_{\theta} u_{1}, \dot{u}\right)=\left(\left(\mathrm{Id}-\mathcal{P}_{\perp}\right) \dot{u} \cdot \nabla \lambda\right)\left(\Sigma_{0} \partial_{\theta} u_{1}, \mathcal{P}_{\perp} \dot{u}\right)
$$

où $\mathcal{P}_{\perp}$ est le projecteur orthogonal sur $\operatorname{ker} \Sigma\left(\bar{u}_{0}, \partial_{x} \varphi\right)$. On voit donc que ce terme n'est pas de signe constant, sauf s'il est nul. Comme la matrice $\Sigma_{0}$ est définie positive, si $\partial_{\theta} u_{1} \neq 0$, en choisissant $\mathcal{P}_{\perp} \dot{u}=\partial_{\theta} u_{1}$, on voit que cette contribution est nulle si et seulement si $\left(\operatorname{Id}-\mathcal{P}_{\perp}\right) \dot{u}$. $\nabla \lambda=0$ pour tout $\dot{u}$. Compte tenu de l'hypothèse 2.2 , cela équivaut à :

$$
\nabla_{u} \lambda\left(\bar{u}_{0}, \partial_{x} \varphi\right)=0, \quad \forall(t, x) \in[0, T] \times \mathbb{R}^{d} .
$$

Cette condition n'est qu'exceptionnellement vérifiée. Notons toutefois que (3.6) est satisfaite si la valeur propre $\lambda$ est indépendante de $u$ ou si $\bar{u}_{0}=\underline{u}$ est une constante, $\partial_{x} \varphi=\underline{\xi}$ est constante et $\nabla \lambda(\underline{u}, \underline{\xi})=0$. En résumé, on retient :

Proposition 3.2. - Si $\Sigma_{0}$ est un bon symétriseur, la condition $D=0$ est satisfaite si et seulement si $\left(\bar{u}_{0}, \partial_{x} \varphi\right)$ vérifie (3.6).

On donnera au paragraphe 5.2 des exemples de systèmes non linéaires admettant un bon symétriseur et vérifiant (3.6).

La démonstration du théorème 3.2 repose, pour l'obtention d'estimations $H^{s}$ et la preuve de la stabilité non linéaire, sur une réduction du linéarisé à une forme modèle. L'hypothèse 2.2 de dégénérescence linéaire de $\lambda$ implique que les matrices $A_{1}$ et $B_{1}$, et donc $D$, ont une structure particulière (lemme 5.1). On peut aussi simplifier l'écriture du linéarisé en introduisant un changement d'inconnues de la forme :

$$
\check{u}=\left(\operatorname{Id}+\sqrt{\varepsilon} \phi_{1}\right) \dot{u} .
$$

Alors, l'opérateur $\check{\mathcal{L}}_{c}^{\varepsilon}=\left(\operatorname{Id}+\sqrt{\varepsilon^{t}} \phi_{1}\right) \mathcal{L}_{c}^{\varepsilon}\left(\operatorname{Id}+\sqrt{\varepsilon} \phi_{1}\right)$ a la même forme que $\mathcal{L}_{c}^{\varepsilon}$. On montre (proposition 5.1) que l'on peut choisir $\phi_{1}$ de sorte que $\check{\mathcal{L}}_{c}^{\varepsilon}$ prenne la forme simplifiée

$$
\check{\mathcal{L}}_{s}^{\varepsilon}=\check{R}^{\varepsilon}+\frac{1}{\varepsilon} A_{0} \partial_{\theta}+\frac{1}{\sqrt{\varepsilon}} \check{B}_{1}
$$

modulo un terme $\check{C}^{\varepsilon}$ uniformément borné. En outre, $\check{B}_{1}$ est symétrique. À l'issue de ces manipulations, on a éliminé la dépendance en $\theta$ du coefficient de la dérivée $\frac{1}{\varepsilon} \partial_{\theta}$. La singularité 
en $\varepsilon$ se trouve reportée au niveau de la contribution d'ordre zéro $\frac{1}{\sqrt{\varepsilon}} \check{B}_{1}$. En outre, le changement d'inconnues (3.7) laisse invariante la matrice $D$ et on a :

$$
D=\check{D}=\frac{1}{2}\left(\check{B}_{1}+{ }^{t} \check{B}_{1}\right)=\check{B}_{1} .
$$

Lorsque $D=0$, on voit que le terme singulier $\check{B}_{1}$ disparait lui aussi. On peut alors reprendre l'analyse menée dans $[3,11,12]$ pour construire des solutions exactes correspondant à des données initiales préparées.

\subsection{Instabilité hyperbolique}

La condition $D=0$ est suffisante pour garantir la stabilité du linéarisé $\mathcal{L}_{c}^{\varepsilon}$. L'étude de cette stabilité peut être affinée par une analyse des interactions d'oscillations à l'échelle $\varepsilon$ entre l'oscillation principale portée par $u_{1}$ et des oscillations de plus petites amplitudes portées par la perturbation $\dot{u}$. L'idée est de faire interagir ces oscillations à l'échelle de temps normale de l'optique géométrique non linéaire qui est ici $\sqrt{\varepsilon}$.

On travaille dans un cadre simplifié. La fonction $\bar{u}_{0}$ est une constante $\underline{u}$. La phase

$$
\varphi(t, x)=\underline{\tau} t+\underline{\xi} \cdot x
$$

est plane, avec $\underline{\xi} \neq 0$. L'équation eikonale (2.11) implique $\underline{\tau}=-\lambda(\underline{u}, \underline{\xi})$. Le terme principal de l'oscillation forte $u_{1}$ est une fonction à valeurs réelles, vérifiant $\partial_{\theta} u_{1} \not \equiv 0$ et $\bar{u}_{1} \equiv 0$.

On se donne une phase auxiliaire $\psi(t, x)=\tau t+\xi \cdot x$. On rend apparentes les oscillations à l'échelle $\varepsilon$ dans la direction $d \psi$ et pour une échelle de temps en $\sqrt{\varepsilon}$, en faisant agir $\mathcal{L}_{c}^{\varepsilon}$ sur des expressions de la forme :

$$
\dot{u}(t, x, \theta)=\check{u}\left(\frac{t}{\sqrt{\varepsilon}}, x, \theta, \frac{\psi}{\varepsilon}\right) .
$$

Ici $\breve{u}(s, x, \theta, \omega)$ est une fonction périodique en les variables $\theta$ et $\omega$. On calcule $\sqrt{\varepsilon} \mathcal{L}_{c}^{\varepsilon} \dot{u}$. On obtient une contribution qui s'écrit

$$
\check{\mathcal{L}} \check{u}=\frac{1}{\sqrt{\varepsilon}} \underline{S}\left(d \varphi \partial_{\theta}+d \psi \partial_{\omega}\right) \check{u}+\left(\underline{\Sigma}_{0} \partial_{s}+G\right) \check{u}+\sqrt{\varepsilon} R \check{u}
$$

où $R$ désigne un opérateur hyperbolique symétrique,

$$
\underline{S}(\tau, \xi)=S(\underline{u},(\tau, \xi)), \quad \underline{\Sigma}_{0}=\Sigma_{0}(\underline{u}),
$$

et $G$ est un opérateur en $(\theta, \omega)$, dont les coefficients dépendent des paramètres $(t, x)$ et aussi de $\theta$ par l'intermédiaire de $u_{1}$ :

$$
G\left(\partial_{\theta}, \partial_{\omega}\right) \check{u}=u_{1} \cdot \nabla_{u} S\left(\underline{u}, d \varphi \partial_{\theta}+d \psi \partial_{\omega}\right) \check{u}+\check{u} \cdot \nabla_{u} S(\underline{u}, d \varphi) \partial_{\theta} u_{1} .
$$

L'intervention de $\check{\mathcal{L}}$ met en jeu un terme singulier en $\varepsilon^{-1 / 2}$, qui force la polarisation des données dans le noyau de $\underline{S}\left(d \varphi \partial_{\theta}+d \psi \partial_{\omega}\right)$. On introduit les fréquences caractéristiques :

$$
Z:=\left\{(\alpha, \beta) \in \mathbb{Z}^{2} ; \operatorname{det} \underline{S}(\alpha d \varphi+\beta d \psi)=0\right\} .
$$


À tout couple $(\alpha, \beta)$ dans $\mathbb{Z}^{2}$ est associé le projecteur orthogonal $P_{\alpha, \beta}$ sur $\operatorname{ker} \underline{S}(\alpha d \varphi+\beta d \psi)$. Les $P_{\alpha, \beta}$ servent à définir le projecteur (orthogonal) $\mathbb{P}$ sur ker $\underline{S}\left(d \varphi \partial_{\theta}+d \psi \partial_{\omega}\right)$ :

$$
\mathbb{P}\left(\sum u_{\alpha, \beta} e^{i(\alpha \theta+\beta \omega)}\right)=\sum P_{\alpha, \beta} u_{\alpha, \beta} e^{i(\alpha \theta+\beta \omega)} .
$$

Le reste $\sqrt{\varepsilon} R$ ne va pas intervenir dans l'étude asymptotique de $\check{\mathcal{L}}$. On peut se concentrer sur l'opérateur :

$$
\mathbb{L}:=\mathbb{P} \underline{\Sigma}_{0} \mathbb{P} \partial_{s}+\mathbb{G}, \quad \mathbb{G}:=\mathbb{P} G \mathbb{P} .
$$

On remarque que $G$ et $\mathbb{G}$ sont à coefficients constants en $\omega$. Ainsi les opérations précédentes se prêtent bien à une décomposition en séries de Fourier en $\omega$. Pour $\beta$ fixé dans $\mathbb{Z}$, on pose :

$$
\begin{aligned}
& \mathbb{P}_{\beta}\left(\sum u_{\alpha} e^{i \alpha \theta}\right):=\sum P_{\alpha, \beta} u_{\alpha} e^{i \alpha \theta}, \\
& G_{\beta}\left(\theta, \partial_{\theta}\right):=G\left(\theta, \partial_{\theta}, i \beta\right), \\
& \mathbb{L}_{\beta}:=\mathbb{P}_{\beta} \underline{\Sigma}_{0} \mathbb{P}_{\beta} \partial_{s}+\mathbb{G}_{\beta}, \quad \mathbb{G}_{\beta}:=\mathbb{P}_{\beta} G_{\beta} \mathbb{P}_{\beta} .
\end{aligned}
$$

Exemple 3.1. - On considère l'équation d'Euler $(\mathcal{D})$. On se place en dimension deux d'espace. On note $x=\left(x_{1}, x_{2}\right)$ les coordonnées. Par changement de repère, on se ramène au cas où la vitesse $\underline{v}$ est nulle. On perturbe l'état de base $\underline{u}=(\underline{p}, 0, \underline{s})$. Le profil $u_{1}$ se décompose en $\left(p_{1}^{*}, v_{1}^{*}, s_{1}^{*}\right)$. La valeur propre $\lambda=0$ produit les phases planes caractéristiques $\xi \cdot x$ où $\xi$ parcourt $\mathbb{R}^{2} \backslash\{0\}$. On fixe $\xi \neq 0$ et on note $\varphi(t, x)=\xi \cdot x$. Les autres valeurs propres correspondent aux modes acoustiques. Elles conduisent aux phases caractéristiques $\psi(t, x)=\tau t+\xi \cdot x$ avec $\xi \neq 0$ et $\tau^{2}=\underline{c}^{2}|\xi|^{2}$, où $\underline{c}$ est la vitesse du son. Notant $\check{u}=(\check{p}, \check{v}, \check{s})$, l' action de $G$ se réduit à :

$$
G\left(\theta, \partial_{\omega}\right) \check{u}=\left(v_{1}^{*} \cdot \xi\right) \underline{\Sigma}_{0} \partial_{\omega} \check{u}+(\check{v} \cdot \underline{\xi}) \underline{\Sigma}_{0} \partial_{\theta} u_{1} .
$$

3.1.a) Phase $\psi(t, x)=\xi \cdot x$ associée à un mode l.d.g. Par changement de coordonnées, on se ramène à $(\underline{\tau}, \xi)=(0,1,0)$ et on choisit $(\tau, \xi)=(0,0,1)$. On obtient alors $Z=\mathbb{Z}^{2}$. Pour $(\alpha, \beta) \neq(0,0), \bar{P}_{\alpha, \beta}$ est le projecteur orthogonal sur $\mathbb{R}^{t}(0, \nu, 0) \oplus \mathbb{R}^{t}(0,0,1)$ avec

$$
\nu=(-\beta, \alpha) /|(\alpha, \beta)|
$$

Les fonctions $\check{u}$ vérifiant $\check{u}=\mathbb{P} \check{u}$ et $\langle\check{u}\rangle=0$ ont leur composante de pression nulle. Elles sont représentées via leurs composantes de vitesse $\check{v}$ et d'entropie $\check{s}$. La condition $\check{u}=\mathbb{P} \check{u}$ se traduit en $\operatorname{div}_{\theta, \omega} \check{v}=0$. L'équation $\mathbb{L} \breve{u}=0$ est, pour la partie de moyenne nulle, équivalente à :

$$
\left\{\begin{array}{l}
\partial_{s} \check{v}+v_{1}(\theta) \cdot \nabla_{\theta, \omega} \check{v}+\check{v} \cdot \nabla_{\theta, \omega} v_{1}=\nabla_{\theta, \omega} q, \quad \operatorname{div}_{\theta, \omega} \check{v}=0, \\
\partial_{s} \check{s}+v_{1}(\theta) \cdot \nabla_{\theta, \omega} \check{s}+\check{v} \cdot \nabla_{\theta, \omega} s_{1}=0 .
\end{array}\right.
$$

On retrouve ici sur les linéarisés le fait que l'équation incompressible (3.16) s'obtient par optique géométrique à partir de l'équation compressible (cf. [21]). Par ailleurs, l'équation pour la moyenne $\langle\breve{u}\rangle$ n'est autre que $\partial_{s}\langle\breve{u}\rangle=0$. Elle est découplée de (3.16) et, à ce titre, peut être oubliée.

3.1.b) Phase $\psi(t, x)=\tau t+\xi \cdot x$ associée à un mode acoustique. L'ensemble $Z$ regroupe alors les points dont les deux coordonnées sont dans $\mathbb{Z}$, situés sur l'union de trois droites :

$$
Z=\{(\alpha, 0) ; \alpha \in \mathbb{Z}\} \cup\{(0, \beta) ; \beta \in \mathbb{Z}\} \cup\left\{(\alpha, \beta) \in \mathbb{Z}^{2} ; \alpha=-2 \beta(\xi \cdot \underline{\xi}) /|\underline{\xi}|^{2} \in \mathbb{Z}\right\} .
$$

Pour $\beta=0$, les propriétés de transparence fournissent $\mathbb{G}_{0}=0$. Pour $\beta \neq 0$ fixé, il y a au plus deux entiers relatifs $\alpha$ vérifiant $(\alpha, \beta) \in Z$. Le projecteur $\mathbb{P}_{\beta}$ sélectionne au plus deux 
modes de Fourier. L'action de $\mathbb{G}_{\beta}$ est non triviale si et seulement si $2 \beta(\xi \cdot \underline{\xi}) /|\underline{\xi}|^{2}$ est un entier relatif. Dans ce cas, l'équation $\mathbb{L}_{\beta} \check{u}=0$ s'interprète en un système différentiel linéaire posé en dimension deux. Ce système est diagonalisable, à valeurs propres imaginaires pures. Ses solutions restent bornées pour tout temps. Les informations données ci-dessus sont reprises et détaillées à l'occasion de l'exemple 6.1.

Pour tout $\varepsilon>0$ fixé, l'opérateur $\mathcal{L}_{c}^{\varepsilon}$ est un opérateur hyperbolique symétrique habituel et admet une estimation d'énergie de la forme

$$
\|\dot{u}(t)\|_{L^{2}} \leqslant C(\varepsilon, t)\left(\|\dot{u}(0)\|_{L^{2}}+\int_{0}^{t}\left\|\mathcal{L}_{c}^{\varepsilon} \dot{u}\left(t^{\prime}\right)\right\|_{L^{2}} d t^{\prime}\right),
$$

et la manière dont le facteur $C(\varepsilon, t)$ dépend de $\varepsilon$ (et de $t$ ) est bien entendu l'un des points essentiels dans l'analyse de la stabilité (linéaire ou non linéaire) des solutions approchées. Comme nous allons rencontrer plusieurs cas de figure dans l'article, nous fixons pour toute la suite le vocabulaire suivant :

DÉFINITION 3.1. - Étant donnée une fonction croissante $k: \mathbb{R}^{+} \rightarrow \mathbb{R}_{*}^{+}$, nous dirons que la famille $\mathcal{L}_{c}^{\varepsilon}$ admet des estimations $L^{2}$ avec un "facteur de type $k$ ", si pour tout $\left.\left.\varepsilon \in\right] 0,1\right]$ et toute fonction test $\dot{u}$ dans $H^{1}\left([0, T] \times \mathbb{R}^{d} \times \mathbb{T}\right)$, on a pour tout $t \in[0, T]$ :

$$
\|\dot{u}(t)\|_{L^{2}} \leqslant k(t / \sqrt{\varepsilon})\left(\|\dot{u}(0)\|_{L^{2}}+\int_{0}^{t}\left\|\mathcal{L}_{c}^{\varepsilon} \dot{u}\left(t^{\prime}\right)\right\|_{L^{2}} d t^{\prime}\right) .
$$

Dans le cas où le facteur $k$ est constant, on dira que la famille $\mathcal{L}_{c}^{\varepsilon}$ est "uniformément stable dans $L^{2}$ ". Lorsque $k$ est polynomial, on parlera alors de "stabilité polynomiale". Nous rencontrerons également des cas où le facteur $k$ est de type exponentiel, $k(s)=a e^{b s}, b>0$. Dans ce cas, nous verrons qu'il peut se produire que la solution approchée soit non linéairement instable.

Sans nuire à la généralité, quitte à effectuer un changement linéaire d'inconnue, on peut supposer que $\underline{\Sigma}_{0}=\mathrm{Id}$. Les solutions de $\mathbb{L} \check{u}=0$ sont alors décrites par le semi-groupe $e^{-s \mathbb{G}}$. On procède à des estimations d'énergie sur $\mathbb{L}$, qui font apparaître :

$$
\operatorname{Re}(\mathbb{G} \check{u}, \check{u})=\operatorname{Re}(G \mathbb{P} \check{u}, \mathbb{P} \check{u})=(D \mathbb{P} \check{u}, \mathbb{P} \check{u})=\mathrm{O}\left(\|\check{u}\|^{2}\right)
$$

où $D$ est précisément la matrice introduite en (3.2). On constate ainsi que l'opérateur $e^{-s \mathbb{G}}$ est continu sur $L^{2}$ avec plus précisément :

$$
g(s):=\left\|e^{-s \mathbb{G}}\left|\left\|\leqslant c_{1} e^{c_{2} s}, \quad\right\| T\right|\right\|:=\sup _{\|u\|_{L^{2}} \leqslant 1}\|T u\|_{L^{2}}
$$

Il se trouve que la fonction $g$ est contrôlée par le facteur $k$ des estimations $L^{2}$ de (3.18).

THÉORÈmE 3.3. - Si la famille $\mathcal{L}_{c}^{\varepsilon}$ admet des estimations $L^{2}$ avec un facteur de type $k$, alors

$$
g(s) \leqslant k(s), \quad \forall s \in \mathbb{R}^{+},
$$

ce que l'on note $g \preceq k$. 
Autrement dit le comportement asymptotique en temps des solutions des équations de modulation obtenues en pratiquant une optique géométrique bi-phase sur le linéarisé $\mathcal{L}_{c}^{\varepsilon}$ est un révélateur de la nature des estimations d'énergie qu'il est possible d'espérer $\operatorname{sur} \mathcal{L}_{c}^{\varepsilon}$. On applique ce principe aux divers cas de figure qui ont été rencontrés.

Exemple 3.2. - Stabilité uniforme. Lorsque $D=0$, l'action de $G$ se simplifie conformément à :

$$
G \check{u}=u_{1} \cdot \nabla_{u} S\left(\underline{u}, d \psi \partial_{\omega}\right) \check{u} .
$$

Comme le profil $u_{1}$ ne dépend pas de $\omega$ et comme le symbole $u_{1} \cdot \nabla_{u} S(\underline{u}, \beta d \psi)$ est symétrique, on reconnaît en $G$ un opérateur antisymétrique. Il existe donc une constante $c>0$ telle que

$$
g(s) \leqslant c, \quad \forall s \in \mathbb{R} .
$$

Dès lors, on s'attend à pouvoir démontrer la stabilité uniforme. C'est ce que confirme notre théorème 3.2 .

Pour la dynamique des gaz, on a $D \not \equiv 0$. L'analyse de l'exemple 3.1.b) indique que les phases $\psi$ portées par les modes acoustiques conduisent à des fonctions $g$ qui sont dans $L^{\infty}\left(\mathbb{R}^{+}\right)$. Seule la situation 3.1.a) est susceptible de donner lieu à une fonction $g$ non bornée sur $\mathbb{R}^{+}$. Cette discussion se trouve donc reportée au niveau du système (3.16).

Exemple 3.3 (Stabilité polynomiale). - On regarde ce que devient (3.16) lorsque l'oscillation forte est portée par l'entropie. On impose $u_{1}=\left(0,0, s_{1}\right)$. On a alors la simplification :

$$
\left\{\begin{array}{l}
\partial_{s} \check{v}=0, \quad \operatorname{div}_{\theta, \omega} \check{v}=0 \\
\partial_{s} \check{s}+\check{v}_{1} \partial_{\theta} s_{1}=0
\end{array}\right.
$$

La composante $\check{v}_{1}(s, \theta)=\check{v}_{1}(0, \theta)$ est déterminée par la première équation. On choisit $\check{v}_{1}(0, \cdot) \not \equiv 0$. L'équation sur $\check{s}$ met alors en jeu le terme source $\check{v}_{1} \partial_{\theta} s_{1} \not \equiv 0$ connu. La composante $\check{s}$ grandit linéairement en $s$. Il existe donc des constantes $>0$, disons $a, b, c, d$, telles que

$$
a s+b \leqslant g(s) \leqslant c s+d, \quad \forall s \geqslant 0 .
$$

D'une part la famille $\mathcal{L}_{c}^{\varepsilon}$ ne peut pas être uniformément stable dans $L^{2}$. D'autre part le comportement en $s$ de $g$ est exempt de croissance exponentielle. On parle alors de stabilité faible du linéarisé $\mathcal{L}_{c}^{\varepsilon}$. La taille à un instant $t>0$ de la norme $L^{2}$ des solutions s'obtient en multipliant la norme $L^{2}$ des données initiales par le facteur singulier $\varepsilon^{-1 / 2}$. On peut espérer compenser cette perte en jouant sur la petitesse du reste $\mathcal{L}_{c}^{\varepsilon} u_{a}^{\varepsilon}$. La construction de solutions exactes associées aux $u_{a}^{\varepsilon}$ reste envisageable. Concrètement, on cherche des estimations uniformes en pondérant les composantes du vecteur $u$ par des puissances différentes du paramètre $\varepsilon$. Par un changement d'inconnues singulier en $\varepsilon$, on se ramène à des équations linéairement et non linéairement stables pour les nouvelles inconnues. Ce programme est mené à son terme dans notre article [4] dans le cas des oscillations d'entropie. Une étape préliminaire consiste à construire des oscillations fortes qui restent polarisées sur l'entropie : cette question est examinée au paragraphe 4.4, consacré aux interactions entre modes l.d.g.

Exemple 3.4 (Instabilité exponentielle). - Pour les équations d'Euler, lorsque l'oscillation forte est polarisée sur la vitesse, la discussion s'organise autrement. L'analyse spectrale de (3.16) pour $u_{1}={ }^{t}(0, \sin m \theta, 0)$ est détaillée dans [9] où il est prouvé que l'opérateur $\mathbb{G}$ a un spectre absolument continu imaginaire pur et des valeurs propres isolées, de partie réelle non nulle, 
symétriques par rapport à l'axe imaginaire. En particulier, il existe un vecteur propre $u$ dans $L^{2}$ vérifiant :

$$
\mathbb{G} u=\mu u \neq 0, \quad \Re e \mu<0 .
$$

On en déduit qu'il existe des constantes $a>0, \lambda>0$ telles que

$$
g(s) \geqslant a e^{\lambda s}, \quad \forall s>0 .
$$

D'après le théorème 3.3 , la famille $\mathcal{L}_{c}^{\varepsilon}$ ne peut pas être polynomialement stable dans $L^{2}$. La construction BKW livre des solutions approchées à un $\mathrm{O}\left(\varepsilon^{m}\right)$ près, avec $m$ arbitraire. Cela ne suffit pas pour compenser l'amplification en $e^{t / \sqrt{\varepsilon}}$ sous-jacente. C'est pourquoi, dans la suite, on parlera d'instabilité exponentielle.

Comme $\mathbb{G}$ est la somme directe orthogonale des $\mathbb{G}_{\beta}$, on a :

$$
g_{\beta}(s) \leqslant g(s), \quad \forall s \in \mathbb{R}^{+} \text {avec } g_{\beta}(s):=\left|\| e^{-s \mathbb{G}_{\beta}}\right||| .
$$

La transparence implique que pour $\beta=0$, on a toujours :

$$
P_{\alpha, 0} D P_{\alpha, 0}=\mathcal{P} D \mathcal{P}=0, \quad \forall \alpha \in \mathbb{Z} .
$$

C'est-à-dire $\mathbb{G}_{0}=0$ et donc $g_{0} \equiv 1$. Cette propriété est prouvée au lemme 5.1 et se voit bien sur la forme matricielle donnée en (5.16). Pour $\beta \neq 0$ le calcul (3.19) effectué sur $\mathbb{G}_{\beta}$ fait apparaître les matrices $P_{\alpha, \beta} D P_{\alpha, \beta}$ où $\alpha$ parcourt $\mathbb{Z}$. En général, lorsque $D \neq 0$, on s'attend à pouvoir ajuster $\psi$ et $\beta$ de façon à ce que les $P_{\alpha, \beta} D P_{\alpha, \beta}$ soient non tous nuls. Il est alors vraisemblable que les semi-groupes $e^{-s \mathbb{G}_{\beta}}$ et donc $e^{-s \mathbb{G}}$ aient un taux de croissance exponentielle, en $e^{c_{2} s}$ avec $c_{2}>0$.

Si tel est le cas, il convient d'examiner les conséquences d'une telle instabilité linéaire sur le problème non linéaire. Au paragraphe 6.2, en renforçant un peu les hypothèses et en suivant les méthodes de [10] et [15], on montre que la forte instabilité linéaire induit une instabilité non linéaire :

THÉORÈME 3.4. - Sous l'hypothèse 6.1, la solution asymptotique $u_{a}^{\varepsilon}$ donnée par le théorème 3.1 est non linéairement instable au sens de la définition 2.3.

\section{Solutions BKW}

Ce paragraphe est consacré à la démonstration du théorème 3.1. Avant de commencer les calculs, on précise les propriétés de transparence impliquées par l'hypothèse 2.2.

\subsection{Transparence quasi-linéaire conservative}

On part de l'identité :

$$
\sum_{j=1}^{d} \xi_{j} f_{j}^{\prime}(u) \Pi(u, \xi)-\lambda(u, \xi) f_{0}^{\prime}(u) \Pi(u, \xi)=0 .
$$

Avec les notations du paragraphe 2.3, ceci s'écrit :

$$
L_{1}(u, \xi) \Pi(u, \xi)=0 .
$$


En dérivant $k-1$ fois (4.1) par rapport à $u$, on obtient une relation qui lie l'application $k$-linéaire $L_{k}(\cdot, \ldots, \cdot, \Pi \cdot)$ aux expressions $L_{j}$ et aux dérivées $D_{u}^{j} \Pi$ et $D_{u}^{j} \lambda$ pour $j<k$. Sous l'hypothèse 2.2, le calcul se simplifie pour faire apparaitre une forme nulle appelée condition de transparence. On explicite ces relations pour $k=2$ et 3 . Dans les énoncés ci-dessous, $(u, \xi) \in \mathcal{U} \times \mathbb{R}^{d} \backslash\{0\}$ est fixé et on note $\Pi=\Pi(u, \xi), L_{k}=L_{k}(u, \xi)$ etc.

LEMME 4.1. - Pour tout $\left(v_{1}, v_{2}\right)$ dans $\mathbb{R}^{2 N}$, on $a$ :

$$
\begin{gathered}
2 \Pi^{\prime} L_{2}\left(v_{1}, \Pi v_{2}\right)=\left(v_{1} \cdot \nabla_{u} \lambda\right) \Pi^{\prime} \Pi v_{2}, \\
\Pi^{\prime} L_{2}\left(\Pi v_{1}, \Pi v_{2}\right)=0 .
\end{gathered}
$$

Démonstration. - En dérivant par rapport à $u$ dans la direction $v_{1}$, l'identité (4.1) appliquée à $v_{2}$ puis en multipliant par $f_{0}^{\prime-1}$, on récupère l'identité :

$$
2 L_{2}\left(v_{1}, \Pi v_{2}\right)=\left(v_{1} \cdot \nabla_{u} \lambda\right) \Pi v_{2}-L_{1}\left(v_{1} \cdot \nabla_{u} \Pi\right) v_{2} .
$$

Comme $\Pi^{\prime} L_{1}=0$, l'identité (4.2) en résulte. Par ailleurs, pour $v_{1}=\Pi v_{1}$, l'hypothèse 2.3 implique que $v_{1} \nabla_{u} \lambda=0$ et $(\operatorname{tr} 2)$ suit.

En composant (4.3) à gauche par l'inverse partiel $Q$, on obtient :

$$
2 Q L_{2}\left(v_{1}, \Pi v_{2}\right)=-(\operatorname{Id}-\Pi)\left(v_{1} \cdot \nabla_{u} \Pi\right) v_{2} .
$$

En dérivant l'identité $\Pi \circ \Pi=\Pi$ dans la direction $v$, il vient

$$
(\operatorname{Id}-\Pi)\left(v \cdot \nabla_{u} \Pi\right)=\left(v \cdot \nabla_{u} \Pi\right) \Pi .
$$

Par définition, $L_{2}$ s'exprime à l'aide des dérivées secondes des flux $f_{j}$ et est donc une application bilinéaire symétrique. En utilisant la notation $\Gamma_{2}$ du paragraphe 2.3, on obtient que :

$$
\begin{aligned}
\Gamma_{2}\left(\Pi w_{1}, \Pi w_{2}\right) & :=-2 Q L_{2}\left(\Pi w_{1} \Pi w_{2}\right) \\
& =\left(\Pi w_{1} \cdot \nabla_{u} \Pi\right) \Pi w_{2}=\left(\Pi w_{2} \cdot \nabla_{u} \Pi\right) \Pi w_{1} .
\end{aligned}
$$

Lemme 4.2. - Pour tout $\left(v_{1}, v_{2}, v_{3}\right)$ dans $\mathbb{R}^{3 N}$, on $a$ :

$$
\begin{aligned}
& 3 \Pi^{\prime} L_{3}\left(\Pi v_{1}, \Pi v_{2}, \Pi v_{3}\right)+\Pi^{\prime} L_{2}\left(\Pi v_{1}, \Gamma_{2}\left(\Pi v_{2}, \Pi v_{3}\right)\right) \\
& \quad+\Pi^{\prime} L_{2}\left(\Pi v_{2}, \Gamma_{2}\left(\Pi v_{1}, \Pi v_{3}\right)\right)+\Pi^{\prime} L_{2}\left(\Pi v_{3}, \Gamma_{2}\left(\Pi v_{1}, \Pi v_{2}\right)\right)=0 .
\end{aligned}
$$

Démonstration. - Les identités (4.3) et (4.5) impliquent :

$$
2 L_{2}\left(\Pi v_{1}, \Pi v_{2}\right)+L_{1} \Gamma_{2}\left(\Pi v_{1}, \Pi v_{2}\right)=0 .
$$

On multiplie cette égalité à gauche par $f_{0}^{\prime}(u)$ pour obtenir :

$$
\begin{aligned}
& \sum_{j=1}^{d} \xi_{j} f_{j}^{\prime \prime}(u)\left(\Pi v_{1}, \Pi v_{2}\right)-\lambda(u, \xi) f_{0}^{\prime \prime}(u)\left(\Pi v_{1}, \Pi v_{2}\right) \\
& \quad+\left(\sum_{j=1}^{d} \xi_{j} f_{j}^{\prime}(u)-\lambda(u, \xi) f_{0}^{\prime}(u)\right) \Gamma_{2}(u, \xi)\left(\Pi v_{1}, \Pi v_{2}\right)=0 .
\end{aligned}
$$

$4^{\mathrm{e}}$ SÉRIE - TOME $36-2003-\mathrm{N}^{\circ} 5$ 
Dans cette expression, $\Pi=\Pi(u, \xi)$. On la dérive par rapport à $u$ dans la direction $h$ puis on multiplie l'identité obtenue à gauche par $f_{0}^{\prime}(u)^{-1}$ :

$$
\begin{aligned}
& 6 L_{3}\left(\Pi v_{1}, \Pi v_{2}, h\right)+2 L_{2}\left(\left(h \cdot \nabla_{u} \Pi\right) v_{1}, \Pi v_{2}\right)+2 L_{2}\left(\Pi v_{1},\left(h \cdot \nabla_{u} \Pi\right) v_{2}\right) \\
& \quad+2 L_{2}\left(h, \Gamma_{2}\left(\Pi v_{1}, \Pi v_{2}\right)\right)+L_{1} F_{1}-\left(h \cdot \nabla_{u} \lambda\right) F_{2}=0 .
\end{aligned}
$$

On teste contre $h=\Pi v_{3}$ et on projette à gauche selon $\Pi^{\prime}$. Ces opérations ont pour effet d'annuler les contributions $L_{1} F_{1}$ et $\left(h \cdot \nabla_{u} \lambda\right) F_{2}$. Comme $v_{1}=\Pi v_{1}$ et $v_{2}=\Pi v_{2}$, il reste :

$$
\begin{aligned}
& 6 \Pi^{\prime} L_{3}\left(\Pi v_{1}, \Pi v_{2}, \Pi v_{3}\right)+2 \Pi^{\prime} L_{2}\left(\left(\Pi v_{3} \cdot \nabla_{u} \Pi\right) \Pi v_{1}, \Pi v_{2}\right) \\
& \quad+2 \Pi^{\prime} L_{2}\left(\Pi v_{1},\left(\Pi v_{3} \cdot \nabla_{u} \Pi\right) v_{2}\right)+2 \Pi^{\prime} L_{2}\left(\Pi v_{3}, \Gamma_{2}\left(\Pi v_{1}, \Pi v_{2}\right)\right)=0 .
\end{aligned}
$$

On utilise (4.5) pour exprimer $\left(\Pi v_{3} \cdot \nabla_{u} \Pi\right) \Pi v_{1}$ et $\left(\Pi v_{3} \cdot \nabla_{u} \Pi\right) \Pi v_{2}$ à l'aide de $\Gamma_{2}\left(\Pi v_{1}, \Pi v_{3}\right)$ et $\Gamma_{2}\left(\Pi v_{2}, \Pi v_{3}\right)$. En tenant compte de la symétrie de $L_{2}$ et $\Gamma_{2}$, on trouve l'identité (tr3).

\subsection{Construction des solutions approchées}

On se donne une solution $\bar{u}_{0}$ de $(\dot{\mathcal{S}})$ dans $H^{\infty}\left([0, T] \times \mathbb{R}^{d}\right)$. On suppose d'abord que

$$
\bar{u}_{1}(t, x)=0, \quad \forall(t, x) \in[0, T] \times \mathbb{R}^{d} .
$$

La phase $\varphi$ est solution de (2.11) et la phase $\varphi^{1}$ est nulle.

Pour $u_{a}^{\varepsilon}$ de la forme (2.6) (avec $n_{1}=\infty$ ) on a

$$
f_{j}\left(u_{a}^{\varepsilon}(t, x, \theta)\right) \sim \sum_{n=0}^{+\infty}(\sqrt{\varepsilon})^{n} f_{j, n}(t, x, \theta)
$$

avec :

$$
\begin{aligned}
& f_{j, 0}(t, x)=f_{j}\left(\bar{u}_{0}(t, x)\right), \quad \forall j \in\{0, \ldots, d\}, \\
& f_{j, n}:=\sum_{k=1}^{n} \frac{1}{k !} \sum_{p_{1}+\cdots+p_{k}=n}\left(D_{u}^{k} f_{j}\right)\left(\bar{u}_{0}\right)\left(u_{p_{1}}, \ldots, u_{p_{k}}\right), \quad \forall n \in \mathbb{N}^{*} .
\end{aligned}
$$

On en déduit que

$$
\mathcal{S}\left(u_{a}^{\varepsilon}, \partial_{t, x, \theta}\right) u_{a}^{\varepsilon} \sim \Sigma\left(u_{a}^{\varepsilon}\right) f_{0}^{\prime}\left(\bar{u}_{0}\right) \sum_{n=-1}^{+\infty}(\sqrt{\varepsilon})^{n} F_{n}, \quad F_{n}=F_{n}^{1}+\partial_{\theta} F_{n+2}^{2}
$$

avec :

$$
F_{n}^{1}:=\sum_{j=0}^{d} f_{0}^{\prime}\left(\bar{u}_{0}\right)^{-1} \partial_{j} f_{j, n}, \quad F_{n}^{2}:=\sum_{j=0}^{d} \partial_{j} \varphi f_{0}^{\prime}\left(\bar{u}_{0}\right)^{-1} f_{j, n} .
$$

On a introduit le facteur $f_{0}^{\prime-1}\left(\bar{u}_{0}\right)$ pour retrouver les notations du paragraphe 2.3 et on convient que $F_{-1}^{1}=0$. On obtient une solution approchée à l'ordre $r$ si et seulement si :

$$
F_{n}=F_{n}^{1}+\partial_{\theta} F_{n+2}^{2}=0, \quad \forall n \in\{-1, \ldots, 2 r-1\} .
$$

On sépare $F_{n}$ en sa moyenne et son oscillation, notant que $\partial_{\theta} F_{n+1}^{2}$ est toujours de moyenne nulle. Par ailleurs, utilisant (2.30), on sépare la condition $F_{n}^{*}=0$ en $\mathcal{P}^{\prime} F_{n}^{*}=0$ et $\mathcal{Q} F_{n}^{*}=0$. On 
regroupe alors les conditions de la manière suivante :

$$
\mathcal{Q} F_{n-1}^{*}=0, \quad \mathcal{P}^{\prime} F_{n}^{*}=0, \quad \bar{F}_{n+1}=0 .
$$

Pour $n=-1$, on adopte la convention $F_{-2}^{*}=0$. Si les équations $\left(\mathcal{E}_{n}\right)$ sont satisfaites pour $n \in\{-1, \ldots, 2 r\}$, alors $F_{n}=0$ pour $n \leqslant 2 r-1$, et $u_{a}^{\varepsilon}$ est solution approchée à l'ordre $r$. On montre d'abord que $\left(\mathcal{E}_{-1}\right)$ est toujours satisfaite et que $\left(\mathcal{E}_{0}\right)$ et $\left(\mathcal{E}_{1}\right)$ déterminent $u_{1}^{*}$ et $\bar{u}_{2}$. On donnera ensuite l'argument général de la récurrence.

\subsubsection{Analyse des premiers termes}

L'équation $\left(\mathcal{E}_{-1}\right)$. Avec les notations du paragraphe 2.3, on a :

$$
F_{-1}^{*}=\partial_{\theta} F_{1}^{2}=\mathcal{L}_{1} \partial_{\theta} u_{1}, \quad \bar{F}_{0}^{1}=\sum_{j=0}^{d} f_{0}^{\prime}\left(\bar{u}_{0}\right)^{-1} f_{j}^{\prime}\left(\bar{u}_{0}\right) \partial_{j} \bar{u}_{0}
$$

D'une part $F_{-2}^{*}=0$ et $\mathcal{P}^{\prime} \mathcal{L}_{1}=0$. D'autre part $\bar{u}_{0}$ est solution de l'équation $(\dot{\mathcal{S}})$. On voit ainsi que l'équation $\left(\mathcal{E}_{-1}\right)$ est satisfaite.

L'équation $\left(\mathcal{E}_{0}\right)$. Comme $\mathcal{Q} \mathcal{L}_{1}=\mathrm{Id}-\mathcal{P}$, l'équation $\mathcal{Q} F_{-1}^{*}=0$ s'écrit $(\mathrm{Id}-\mathcal{P}) \partial_{\theta} u_{1}=0$, et est donc équivalente à la condition de polarisation :

$$
(\mathrm{Id}-\mathcal{P}) u_{1}^{*}=0
$$

Par ailleurs :

$$
\begin{aligned}
& F_{0}^{*}=\partial_{\theta} F_{2}^{2}=\mathcal{L}_{1} \partial_{\theta} u_{2}+\partial_{\theta} \mathcal{L}_{2}\left(u_{1}, u_{1}\right), \\
& \bar{F}_{1}=\bar{F}_{1}^{1}=\sum_{j=0}^{d} f_{0}^{\prime}\left(\bar{u}_{0}\right)^{-1} \partial_{j}\left(f_{j}^{\prime}\left(\bar{u}_{0}\right) \bar{u}_{1}\right) .
\end{aligned}
$$

La seconde équation est de toute évidence vérifiée puisqu'on a pris $\bar{u}_{1}=0$. Si $u_{1}^{*}$ satisfait $\left(\mathcal{Z}_{0}^{\prime}\right)$, alors la condition de transparence (tr2) du lemme 4.1 implique que

$$
\mathcal{P}^{\prime} F_{0}^{*}=\partial_{\theta} \mathcal{P}^{\prime} \mathcal{L}_{2}\left(\mathcal{P} u_{1}^{*}, \mathcal{P} u_{1}^{*}\right)=0
$$

En conclusion, $\left(\mathcal{E}_{0}\right)$ se réduit à la condition de polarisation $\left(\mathcal{Z}_{0}^{\prime}\right)$ que l'on suppose dorénavant satisfaite.

L'équation $\left(\mathcal{E}_{1}\right)$. L'équation $\mathcal{Q} F_{0}^{*}=0$ s'écrit :

$$
(\operatorname{Id}-\mathcal{P}) \partial_{\theta} u_{2}=-\partial_{\theta} \mathcal{Q} \mathcal{L}_{2}\left(u_{1}, u_{1}\right) .
$$

Comme $\partial_{\theta}$ est injective sur les fonctions à moyenne nulle et que $u_{1}=u_{1}^{*}$, elle équivaut donc à :

$$
(\operatorname{Id}-\mathcal{P}) u_{2}^{*}=-\mathcal{Q} \mathcal{L}_{2}\left(\mathcal{P} u_{1}^{*}, \mathcal{P} u_{1}^{*}\right)^{*}=\frac{1}{2}\left(\Gamma_{2}\left(\mathcal{P} u_{1}^{*}, \mathcal{P} u_{1}^{*}\right)\right)^{*}
$$

Ensuite, le calcul fournit :

$$
F_{1}^{*}=M u_{1}^{*}+\mathcal{L}_{1} \partial_{\theta} u_{3}+2 \partial_{\theta} \mathcal{L}_{2}\left(u_{1}, u_{2}\right)+\partial_{\theta} \mathcal{L}_{3}\left(u_{1}, u_{1}, u_{1}\right), \quad \bar{F}_{2}=\sum_{j=0}^{d} f_{0}^{\prime}\left(\bar{u}_{0}\right)^{-1} \partial_{j} \overline{f_{j, 2}}
$$


Ici $M$ est le linéarisé de $(\mathcal{S})$ en $\bar{u}_{0}$ :

$$
M v=M\left(t, x, \partial_{t, x}\right) v:=\sum_{j=0}^{d} f_{0}^{\prime}\left(\bar{u}_{0}\right)^{-1} \partial_{j}\left(f_{j}^{\prime}\left(\bar{u}_{0}\right) v\right)
$$

LeMme 4.3. - L'opérateur $\mathcal{P}^{\prime} M \mathcal{P}$ est de la forme $\mathcal{P}^{\prime}\left(X_{0}+E\right) \mathcal{P}$ avec :

$$
X_{0}:=\partial_{t}+\sum_{j=1}^{d} \frac{\partial \lambda}{\partial \xi_{j}}\left(\bar{u}_{0}, \partial_{x} \varphi\right) \partial_{j}, \quad E:=\sum_{j=0}^{d} \mathcal{P} f_{0}^{\prime}\left(\bar{u}_{0}\right)^{-1} \partial_{j}\left(f_{j}^{\prime}\left(\bar{u}_{0}\right) \mathcal{P}\right)
$$

Démonstration. - C'est un résultat classique, qui s'obtient en dérivant en $\xi$ la relation $A(u, \xi) \Pi(u, \xi)=\lambda(u, \xi) \Pi(u, \xi)$, puis en multipliant à gauche par $\Pi^{\prime}(u, \xi)$. Il vient

$$
\Pi^{\prime}(u, \xi) f_{0}^{\prime}(u)^{-1} f_{j}^{\prime}(u) \Pi(u, \xi)=\frac{\partial \lambda}{\partial \xi_{j}}(u, \xi) \Pi^{\prime}(u, \xi) \Pi(u, \xi), \quad \forall j \in\{1, \ldots, d\}
$$

Par définition de $M$ on a :

$$
\begin{aligned}
\mathcal{P}^{\prime} M \mathcal{P} v=\mathcal{P}^{\prime} M \mathcal{P}(\mathcal{P} v)= & \sum_{j=0}^{d} \mathcal{P}^{\prime} f_{0}^{\prime}\left(\bar{u}_{0}\right)^{-1} f_{j}^{\prime}\left(\bar{u}_{0}\right) \mathcal{P} \partial_{j}(\mathcal{P} v) \\
& +\sum_{j=0}^{d} \mathcal{P}^{\prime} f_{0}^{\prime}\left(\bar{u}_{0}\right)^{-1} \partial_{j}\left(f_{j}^{\prime}\left(\bar{u}_{0}\right) \mathcal{P}\right) \mathcal{P} v
\end{aligned}
$$

L'équation $\mathcal{P}^{\prime} F_{1}^{*}=0$ fait apparaître l'opérateur $\mathcal{P}^{\prime} M \mathcal{P}$ agissant sur la composante $u_{1}^{*}=\mathcal{P} u_{1}^{*}$; le terme $\mathcal{P}^{\prime} \mathcal{L}_{1} \partial_{\theta} u_{3}$ s'annule. Comme $u_{1}=u_{1}^{*}=\mathcal{P} u_{1}^{*}$, le lemme 4.1 implique que $\mathcal{P}^{\prime} \mathcal{L}_{2}\left(u_{1}, \mathcal{P} u_{2}^{*}\right)=0$. On a donc :

$$
\partial_{\theta} \mathcal{P}^{\prime} \mathcal{L}_{2}\left(u_{1}, u_{2}\right)=\mathcal{P}^{\prime} \mathcal{L}_{2}\left(\partial_{\theta} u_{1}, \bar{u}_{2}\right)+\partial_{\theta} \mathcal{P}^{\prime} \mathcal{L}_{2}\left(u_{1}^{*},(\operatorname{Id}-\mathcal{P}) u_{2}^{*}\right)
$$

$\operatorname{Par}\left(\mathcal{Z}_{1}^{\prime}\right)$, le dernier terme s'exprime à l'aide de $u_{1}^{*}$. En outre, le lemme 4.2 implique :

$$
\mathcal{P}^{\prime} \mathcal{L}_{3}\left(u_{1}^{*}, u_{1}^{*}, u_{1}^{*}\right)+\mathcal{P}^{\prime} \mathcal{L}_{2}\left(u_{1}^{*}, \Gamma_{2}\left(u_{1}^{*}, u_{1}^{*}\right)\right)=0
$$

On en déduit que l'équation $\mathcal{P}^{\prime} F_{1}^{*}=0$ se réduit à :

$$
\mathcal{P}^{\prime} M \mathcal{P} u_{1}^{*}+2 \mathcal{P}^{\prime} \mathcal{L}_{2}\left(\bar{u}_{2}, \partial_{\theta} \mathcal{P} u_{1}^{*}\right)-\mathcal{P} \mathcal{L}_{2}\left(\overline{\Gamma_{2}\left(u_{1}^{*}, u_{1}^{*}\right)}, \partial_{\theta} \mathcal{P} u_{1}^{*}\right)=0
$$

On utilise l'identité (4.2) pour exprimer les deux derniers termes sous la forme $\left(w \cdot \nabla_{u} \lambda\right) \partial_{\theta} \mathcal{P}^{\prime} \mathcal{P} u_{1}^{*}$. Notant :

$$
\left\{\begin{array}{l}
h_{1}\left(t, x, u_{1}^{*}, \bar{u}_{2}\right):=\bar{u}_{2} \cdot \nabla \lambda+\left\langle\mathcal{Q} \mathcal{L}_{2}\left(\mathcal{P} u_{1}^{*}, \mathcal{P} u_{1}^{*}\right)\right\rangle \cdot \nabla \lambda \\
X=X_{0}+h_{1} \partial_{\theta}
\end{array}\right.
$$

et explicitant les termes $\overline{f_{j, 2}}$ intervenant dans $\bar{F}_{2}$, on a montré le résultat suivant. 
LEMME 4.4. - Pour $u_{1}$ vérifiant (4.6) et $\left(\mathcal{Z}_{0}^{\prime}\right)$, les équations contenues dans $\left(\mathcal{E}_{1}\right)$ sont équivalentes à un système portant $\operatorname{sur}\left(\mathcal{P} u_{1}^{*}, \bar{u}_{2}\right)$ :

$$
\left\{\begin{array}{l}
\mathcal{P}^{\prime}(X+E) \mathcal{P} u_{1}^{*}=0, \\
M \bar{u}_{2}+\frac{1}{2} \sum_{j=0}^{d} f_{0}^{\prime}\left(\bar{u}_{0}\right)^{-1} \partial_{j}\left\langle f_{j}^{\prime \prime}\left(\bar{u}_{0}\right)\left(\mathcal{P} u_{1}^{*}, \mathcal{P} u_{1}^{*}\right)\right\rangle=0
\end{array}\right.
$$

complété par la relation $\left(\mathcal{Z}_{1}^{\prime}\right)$ qui détermine $(\mathrm{Id}-\mathcal{P}) u_{2}^{*}$.

La résolution de $\left(\mathcal{Z}_{1}\right)$ n'est pas immédiate. La première équation est bien un transport pour $\mathcal{P} u_{1}^{*}$, mais elle dépend de $\bar{u}_{2}$ par l'intermédiaire du coefficient de $\partial_{\theta}, h_{1}$. Par contre la seconde fait intervenir les dérivées en $(t, x)$ du terme quadratique moyenné $\left\langle f_{j}^{\prime \prime}\left(\bar{u}_{0}\right)\left(u_{1}^{*}, u_{1}^{*}\right)\right\rangle$. Néanmoins, le problème de Cauchy pour $\left(\mathcal{Z}_{1}\right)$ est bien posé. On note $H_{\text {pol }}^{s}\left(\mathbb{R}^{d} \times \mathbb{T}\right)$ l'espace des données initiales de regularité $H^{s}$ pour $\mathcal{P} u_{1}^{*}$ : il s'agit de l'espace des fonctions $u$ dans $H^{s}\left(\mathbb{R}^{d} \times \mathbb{T}\right)$ à valeurs dans $\mathbb{R}^{N}$, de moyenne nulle et telles que $\mathcal{P}_{\mid t=0} u=u$.

Proposition 4.1.- Soit $s>\frac{3+d}{2}$. Pour toute donnée initiale $\left(\mathcal{P} u_{1,0}^{*}, \bar{u}_{2,0}\right)$ sélectionnée dans $H_{\mathrm{pol}}^{s+1}\left(\mathbb{R}^{d} \times \mathbb{T}\right) \times H^{s}\left(\mathbb{R}^{d}\right)$, le système $\left(\mathcal{Z}_{1}\right)$ admet une unique solution $\left(\mathcal{P} u_{1}^{*}, \bar{u}_{2}\right)$ dans $\mathcal{W}^{s}(T) \times \mathcal{W}^{s}(T)$. La fonction $(\operatorname{Id}-\mathcal{P}) u_{2}^{*}$ définie par $\left(\mathcal{Z}_{1}^{\prime}\right)$ est alors dans $\mathcal{W}^{s}(T)$.

Remarque 4.1. - La perte de régularité des données $H^{s+1}$ vers la solution $\mathcal{W}^{s}$ illustre le caractère "faiblement" hyperbolique de $\left(\mathcal{Z}_{1}\right)$. Par ailleurs, on notera que la solution existe sur l'intervalle $[0, T]$ où sont définis $u_{0}$ et $\varphi$, bien que le problème soit non linéaire.

Démonstration. - On introduit une inconnue auxiliaire $\psi_{1}(t, x)$ solution de l'équation

$$
X_{0} \psi_{1}=h_{1}, \quad \psi_{1}(0, x)=0 .
$$

On fait jouer à $\psi_{1}$ le rôle d'un décalage de phase. On procède au changement de fonction :

$$
v_{1}^{*}(t, x, \theta)=\mathcal{P} u_{1}^{*}\left(t, x, \theta+\psi_{1}(t, x)\right) .
$$

Par construction, la première équation de $\left(\mathcal{Z}_{1}\right)$ est équivalente à :

$$
\mathcal{P}^{\prime}\left(X_{0}+E\right) \mathcal{P} v_{1}^{*}=0, \quad \mathcal{P} v_{1}^{*}=v_{1}^{*}
$$

En outre, pour toute fonction continue $g(t, x, u)$, on a :

$$
\int_{\mathbb{T}} g\left(\mathcal{P} v_{1}^{*}\left(t, x, \theta^{\prime}\right)\right) d \theta^{\prime}=\int_{\mathbb{T}} g\left(\mathcal{P} u_{1}^{*}(t, x, \theta)\right) d \theta .
$$

En particulier, le coefficient $h_{1}$ défini en (4.7) vérifie :

$$
h_{1}\left(t, x, u_{1}^{*}, \bar{u}_{2}\right)=h_{1}\left(t, x, v_{1}^{*}, \bar{u}_{2}\right) .
$$

De même :

$$
\left\langle f_{j}^{\prime \prime}\left(\bar{u}_{0}\right)\left(\mathcal{P} u_{1}^{*}, \mathcal{P} u_{1}^{*}\right)\right\rangle=\left\langle f_{j}^{\prime \prime}\left(\bar{u}_{0}\right)\left(\mathcal{P} v_{1}^{*}, \mathcal{P} v_{1}^{*}\right)\right\rangle .
$$


Il est alors clair que, par le changement d'inconnues (4.9), le système composé de $\left(\mathcal{Z}_{1}\right)$ et (4.8) pour $\left(\mathcal{P} u_{1}^{*}, \bar{u}_{2}, \psi_{1}\right)$ est équivalent au système suivant, portant sur les inconnues $\left(\mathcal{P} v_{1}^{*}, \bar{u}_{2}, \psi_{1}\right)$ :

$$
\left\{\begin{array}{l}
\mathcal{P}^{\prime}\left(X_{0}+E\right) \mathcal{P} v_{1}^{*}=0 \\
M \bar{u}_{2}=-\frac{1}{2} \sum_{j=0}^{d} f_{0}^{\prime}\left(\bar{u}_{0}\right)^{-1} \partial_{j}\left\langle f_{j}^{\prime \prime}\left(\bar{u}_{0}\right)\left(\mathcal{P} v_{1}^{*}, \mathcal{P} v_{1}^{*}\right)\right\rangle, \\
X_{0} \psi_{1}=h_{1}\left(t, x, v_{1}^{*}, \bar{u}_{2}\right) .
\end{array}\right.
$$

Pour résoudre (4.11), on s'organise selon un ordre précis.

On détermine d'abord $v_{1}^{*}=\mathcal{P} v_{1}^{*} \in \mathcal{W}^{s+1}(T)$ comme solution de la première équation avec donnée de Cauchy $v_{1 \mid t=0}^{*}=\mathcal{P} u_{1,0}^{*} \in H^{s+1}$. Il s'agit en effet d'une équation de transport, dans le fibré $\operatorname{ker}(\mathrm{Id}-\mathcal{P})$, et la condition (2.22) implique que le rang de $\mathcal{P}^{\prime} \mathcal{P}$ est bien égal à la dimension de la fibre.

La seconde équation est une equation linéaire en $\bar{u}_{2}$ avec second membre connu dans $\mathcal{W}^{s}(T)$ puisque $s>d+3 / 2$ (on perd au passage une dérivée). Comme le système $M$ est hyperbolique symétrisable, cette équation a une unique solution telle que $\bar{u}_{2 \mid t=0}=\bar{u}_{2,0} \in H^{s}$ et $\bar{u}_{2} \in \mathcal{W}^{s}(T)$.

Ayant déterminé $v_{1}^{*}$ et $\bar{u}_{2}$, le terme source $h_{1}$ de la troisième équation est connu et appartient à $\mathcal{W}^{s}(T)$. On résout alors cette équation avec la donnée initiale $\psi_{1 \mid t=0}=0$ pour trouver $\psi_{1} \in \mathcal{W}^{s}(T)$.

On conclut en vérifiant que $\mathcal{P} u_{1}^{*}$ défini par (4.9) appartient à $\mathcal{W}^{s}(T)$, ce qui est vrai puisque $s$ est assez grand.

\subsubsection{La récurrence}

On montre que le raisonnement tenu pour traiter $\left(\mathcal{E}_{1}\right)$ s'étend aux $\left(\mathcal{E}_{n}\right)$ qui suivent. Notons :

$$
U_{n}=\left(\mathcal{P} u_{n}^{*}, \bar{u}_{n+1},(\mathrm{Id}-\mathcal{P}) u_{n+1}^{*}\right) .
$$

Par construction, on a :

$$
U_{0}=\left(\mathcal{P} u_{0}^{*}, \bar{u}_{1},(\mathrm{Id}-\mathcal{P}) u_{1}^{*}\right)=(0,0,0) .
$$

La proposition 4.1 fournit $U_{1} \in \mathcal{W}^{\infty}(T)$ si les données initiales affectées à $\left(\mathcal{P} u_{1}^{*}, \bar{u}_{2}\right)$ sont dans $H^{\infty}$. On retient que la composante $(\mathrm{Id}-\mathcal{P}) u_{2}^{*}$ est donnée $\operatorname{par}\left(\mathcal{Z}_{1}^{\prime}\right)$.

Lemme 4.5. - Pour $n \geqslant 2$, supposons connus les $U_{k}$, pour $k<n$, dans $\mathcal{W}^{\infty}(T)$. Alors, l'équation $\left(\mathcal{E}_{n}\right)$ est équivalente à un système d'équations pour $U_{n}$ de la forme

$$
\left\{\begin{array}{l}
\mathcal{P}^{\prime}(X+E) \mathcal{P} u_{n}^{*}+\mathcal{P}^{\prime} h_{n} \partial_{\theta} \mathcal{P} u_{1}^{*}+\mathcal{G}_{n-1}=0 \\
M \bar{u}_{n+1}+\sum_{j=0}^{d} f_{0}^{\prime}\left(\bar{u}_{0}\right)^{-1} \partial_{j}\left\langle f_{j}^{\prime \prime}\left(\bar{u}_{0}\right)\left(\mathcal{P} u_{1}^{*}, \mathcal{P} u_{n}^{*}\right)\right\rangle+\mathcal{G}_{n-1}=0
\end{array}\right.
$$

$$
(\mathrm{Id}-\mathcal{P}) u_{n+1}^{*}=-2 \mathcal{Q}\left(\mathcal{L}_{2}\left(\mathcal{P} u_{1}^{*}, \mathcal{P} u_{n}^{*}\right)\right)^{*}+\mathcal{G}_{n-1} .
$$

Le champ de vecteurs $X$ est déterminé en (4.7). On a :

$$
h_{n}:=\left(\bar{u}_{n+1}-\left\langle\Gamma_{2}\left(u_{1}^{*}, \mathcal{P} u_{n}^{*}\right)\right\rangle\right) \cdot \nabla \lambda .
$$

Les $\mathcal{G}_{k}$ désignent différentes expressions qui ne dépendent que de $\left(U_{1}, \ldots, U_{k}\right)$ et de leurs dérivées. 
Démonstration. - Pour $n \geqslant 2$, on a :

$$
F_{n-1}^{*}=\mathcal{L}_{1} \partial_{\theta} u_{n+1}+2 \partial_{\theta} \mathcal{L}_{2}\left(u_{1}, u_{n}\right)+\mathcal{H}_{n-1}
$$

où les $\mathcal{H}_{k}$ sont des expressions qui ne dépendent que de $\left(u_{0}, \ldots, u_{k}\right)$ et de leurs dérivées. En particulier, ce sont des fonction du type $\mathcal{G}_{k}$.

Poussant le développement un cran plus loin, on a, pour $n \geqslant 2$ :

$$
\begin{aligned}
F_{n}^{*}= & M u_{n}^{*}+\mathcal{L}_{1} \partial_{\theta} u_{n+2}+2 \partial_{\theta} \mathcal{L}_{2}\left(u_{1}, u_{n+1}\right)+\iota_{n} \partial_{\theta} \mathcal{L}_{2}\left(u_{2}, u_{n}\right) \\
& +3 \partial_{\theta} \mathcal{L}_{3}\left(u_{1}, u_{1}, u_{n}\right)+\mathcal{H}_{n-1} .
\end{aligned}
$$

Le coefficient $\iota_{n}$ introduit ici vaut 1 si $n=2$ et 2 pour $n>2$. Par ailleurs, on trouve :

$$
\bar{F}_{n+1}=M \bar{u}_{n+1}+\sum_{j=0}^{d} f_{0}^{\prime}\left(\bar{u}_{0}\right)^{-1} \partial_{j}\left\langle f_{j}^{\prime \prime}\left(\bar{u}_{0}\right)\left(u_{1}^{*}, u_{n}^{*}\right)\right\rangle+\mathcal{H}_{n-1} .
$$

La première équation $\mathcal{Q} F_{n-1}^{*}=0$ de $\left(\mathcal{E}_{n}\right)$ s'écrit, en inversant $\partial_{\theta}$ dans l'ensemble des fonctions de moyenne nulle :

$$
(\mathrm{Id}-\mathcal{P}) u_{n+1}^{*}=-2 \mathcal{Q} \mathcal{L}_{2}\left(\mathcal{P} u_{1}^{*}, \mathcal{P} u_{n}^{*}\right)^{*}+\mathcal{G}_{n-1}=\left(\Gamma_{2}\left(\mathcal{P} u_{1}^{*}, \mathcal{P} u_{n}^{*}\right)\right)^{*}+\mathcal{G}_{n-1}
$$

On obtient la forme $\left(\mathcal{Z}_{n}^{\prime}\right)$ annoncée.

De même, l'équation $\bar{F}_{n+1}=0$ conduit directement à la seconde équation de $\left(\mathcal{Z}_{n}\right)$, en décomposant $u_{n}^{*}$ en $\mathcal{P} u_{n}^{*}+(\operatorname{Id}-\mathcal{P}) u_{n}^{*}$.

On analyse maintenant l'équation $\mathcal{P}^{\prime} F_{n}^{*}=0$. On a $\mathcal{P}^{\prime} \mathcal{L}_{1} \partial_{\theta} u_{n+2}=0$ et, d'après le lemme 4.1, $\mathcal{P}^{\prime} \mathcal{L}_{2}\left(\mathcal{P} u_{1}^{*}, \mathcal{P} u_{n+1}^{*}\right)=0$. Par conséquent, il vient :

$$
\begin{aligned}
\mathcal{P}^{\prime} F_{n}^{*}= & \mathcal{P}^{\prime} M \mathcal{P} u_{n}^{*}+2 \partial_{\theta} \mathcal{P}^{\prime} \mathcal{L}_{2}\left(\mathcal{P} u_{1}^{*}, \bar{u}_{n+1}+(\mathrm{Id}-\mathcal{P}) u_{n+1}^{*}\right) \\
& +\iota_{n} \partial_{\theta} \mathcal{P}^{\prime} \mathcal{L}_{2}\left(u_{2}, u_{n}\right)+3 \partial_{\theta} \mathcal{P}^{\prime} \mathcal{L}_{3}\left(\mathcal{P} u_{1}^{*}, \mathcal{P} u_{1}^{*}, \mathcal{P} u_{n}^{*}\right)+\mathcal{G}_{n-1}
\end{aligned}
$$

$\operatorname{Avec}\left(\mathcal{Z}_{n}^{\prime}\right)$, on a :

$$
\begin{aligned}
\mathcal{P}^{\prime} \mathcal{L}_{2}\left(\mathcal{P} u_{1}^{*},(\operatorname{Id}-\mathcal{P}) u_{n+1}^{*}\right)= & \mathcal{P}^{\prime} \mathcal{L}_{2}\left(\mathcal{P} u_{1}^{*}, \Gamma_{2}\left(\mathcal{P} u_{1}^{*}, \mathcal{P} u_{n}^{*}\right)\right) \\
& -\mathcal{P}^{\prime} \mathcal{L}_{2}\left(\mathcal{P} u_{1}^{*}, \overline{\Gamma_{2}\left(\mathcal{P} u_{1}^{*}, \mathcal{P} u_{n}^{*}\right)}\right)+\mathcal{G}_{n-1} .
\end{aligned}
$$

Par le lemme 4.1, on a :

$$
\begin{aligned}
& \mathcal{P}^{\prime} \mathcal{L}_{2}\left(\mathcal{P} u_{1}^{*}, \bar{u}_{n+1}\right)=\mathcal{P}^{\prime}\left(\bar{u}_{n+1} \cdot \nabla \lambda\right) \mathcal{P} u_{1}^{*}, \\
& \mathcal{P}^{\prime} \mathcal{L}_{2}\left(\mathcal{P} u_{1}^{*}, \overline{\Gamma_{2}\left(\mathcal{P} u_{1}^{*}, \mathcal{P} u_{n}^{*}\right)}\right)=\mathcal{P}^{\prime}\left(\left\langle\Gamma_{2}\left(\mathcal{P} u_{1}^{*}, \mathcal{P} u_{n}^{*}\right)\right\rangle \cdot \nabla \lambda\right) \mathcal{P} u_{1}^{*} .
\end{aligned}
$$

Par le lemme 4.2, on a :

$$
3 \mathcal{P}^{\prime} \mathcal{L}_{3}\left(\mathcal{P} u_{1}^{*}, \mathcal{P} u_{1}^{*}, \mathcal{P} u_{n}^{*}\right)=-2 \mathcal{P}^{\prime} \mathcal{L}_{2}\left(\mathcal{P} u_{1}^{*}, \Gamma_{2}\left(\mathcal{P} u_{1}^{*}, \mathcal{P} u_{n}^{*}\right)\right)-\mathcal{P}^{\prime} \mathcal{L}_{2}\left(\mathcal{P} u_{n}^{*}, \Gamma_{2}\left(\mathcal{P} u_{1}^{*}, \mathcal{P} u_{1}^{*}\right)\right)
$$

On sait par ailleurs que :

$$
\mathcal{P}^{\prime} \mathcal{L}_{2}\left(\mathcal{P} u_{2}^{*}, \mathcal{P} u_{n}^{*}\right)=0, \quad u_{n}-\mathcal{P} u_{n}^{*}=\mathcal{G}_{n-1}
$$

Pour calculer le terme $\mathcal{L}_{2}\left(u_{2}, u_{n}\right)$, il convient de distinguer les cas $n=2$ et $n>2$. Pour $n=2$, la composante $\mathcal{P} u_{2}^{*}$ est inconnue. Par contre, pour $n>2, \mathcal{P} u_{2}^{*}$ est identifié. Quelle que soit la 
situation, on a la décomposition :

$$
\iota_{n} \mathcal{P}^{\prime} \mathcal{L}_{2}\left(u_{2}, u_{n}\right)=2 \mathcal{P}^{\prime} \mathcal{L}_{2}\left(\bar{u}_{2}+(\mathrm{Id}-\mathcal{P}) u_{2}^{*}, \mathcal{P} u_{n}^{*}\right)+\mathcal{G}_{n-1}
$$

À l'aide de $\left(\mathcal{Z}_{1}^{\prime}\right)$, on extrait :

$$
(\operatorname{Id}-\mathcal{P}) u_{2}^{*}=\frac{1}{2}\left(\Gamma_{2}\left(\mathcal{P} u_{1}^{*}, \mathcal{P} u_{1}^{*}\right)\right)^{*}=\frac{1}{2} \Gamma_{2}\left(\mathcal{P} u_{1}^{*}, \mathcal{P} u_{1}^{*}\right)+\left\langle\mathcal{Q} \mathcal{L}_{2}\left(\mathcal{P} u_{1}^{*}, \mathcal{P} u_{1}^{*}\right)\right\rangle .
$$

En utilisant le lemme 4.1, il vient :

$$
\iota_{n} \mathcal{P}^{\prime} \mathcal{L}_{2}\left(u_{2}, u_{2}\right)=\mathcal{P}^{\prime} h_{1} \mathcal{P} u_{n}^{*}+\mathcal{P}^{\prime} \mathcal{L}_{2}\left(\Gamma_{2}\left(\mathcal{P} u_{1}^{*}, \mathcal{P} u_{1}^{*}\right), \mathcal{P} u_{n}^{*}\right)+\mathcal{G}_{n-1}
$$

où la fonction $h_{1}=\left(\bar{u}_{2}+\left\langle\mathcal{Q} \mathcal{L}_{2}\left(\mathcal{P} u_{1}^{*}, \mathcal{P} u_{1}^{*}\right)\right\rangle\right) \cdot \nabla \lambda$, définie comme en (4.7), ne dépend que des variables $(t, x)$.

On assemble les renseignements obtenus ci-dessus pour réduire l'équation $\mathcal{P}^{\prime} F_{n}^{*}=0$ sous la forme :

$$
\mathcal{P}^{\prime} M \mathcal{P} u_{n}^{*}+\mathcal{P}^{\prime} h_{1} \partial_{\theta} \mathcal{P} u_{n}^{*}+\mathcal{P}^{\prime} h_{n} \partial_{\theta} \mathcal{P} u_{1}^{*}=\mathcal{G}_{n-1} .
$$

Finalement, on applique le lemme 4.3. On voit que les contraintes figurant dans $\left(\mathcal{E}_{n}\right)$ se ramènent aux systèmes $\left(\mathcal{Z}_{n}\right)$ et $\left(\mathcal{Z}_{n}^{\prime}\right)$. Le lemme 4.5 est démontré.

Il reste à montrer que l'équation $\left(\mathcal{Z}_{n}\right)$ permet de déterminer les composantes $\mathcal{P} u_{n}^{*}$ et $\bar{u}_{n+1}$.

Proposition 4.2. - Pour $n \geqslant 2$, supposons connus les $U_{k}$, pour $k<n$, dans $\mathcal{W}^{\infty}(T)$. Alors, pour toute donnée initiale $\left(\mathcal{P} u_{n, 0}^{*}, \bar{u}_{n+1,0}\right)$ sélectionnée dans l'espace

$$
H_{\mathrm{pol}}^{\infty}\left(\mathbb{R}^{d} \times \mathbb{T}\right) \times H^{\infty}\left(\mathbb{R}^{d}\right)
$$

le système $\left(\mathcal{Z}_{n}\right)$ admet une unique solution $\left(\mathcal{P} u_{n}^{*}, \bar{u}_{n+1}\right)$ dans $\mathcal{W}^{\infty}(T)$. La fonction $(\operatorname{Id}-\mathcal{P}) u_{n+1}^{*}$ définie $\operatorname{par}\left(\mathcal{Z}_{n}^{\prime}\right)$ est dans $\mathcal{W}^{\infty}(T)$.

Démonstration. - On introduit à nouveau une inconnue auxiliaire $\psi_{n}(t, x)$ qui est solution de

$$
X_{0} \psi_{n}=\bar{u}_{n+1} \cdot \nabla \lambda, \quad \psi_{n}(0, x)=0 .
$$

On effectue le changement d'inconnues

$$
w_{n}^{*}(t, x, \theta)=\mathcal{P} w_{n}^{*}(t, x, \theta):=\mathcal{P} u_{n}^{*}(t, x, \theta)+\psi_{n}(t, x) \partial_{\theta} u_{1}^{*}(t, x, \theta) .
$$

On a alors :

$$
\mathcal{P}^{\prime}(X+E) \mathcal{P} w_{n}^{*}=\mathcal{P}^{\prime}(X+E) \mathcal{P} u_{n}^{*}+\mathcal{P}^{\prime}\left(X_{0} \psi_{n}\right) \partial_{\theta} u_{1}^{*}+\psi_{n} \mathcal{P}^{\prime}(X+E) \mathcal{P} \partial_{\theta} u_{1}^{*}
$$

La dérivée $\partial_{\theta}$ commute avec l'opérateur $\mathcal{P}^{\prime}(X+E) \mathcal{P}$ dont les coefficients ne dépendent pas de $\theta$. Par suite, l'équation $\left(\mathcal{Z}_{1}\right)$ implique que le dernier terme est nul. Avec (4.15), la première équation de $\left(\mathcal{Z}_{n}\right)$ équivaut à :

$$
\mathcal{P}^{\prime}(X+E) \mathcal{P} w_{n}^{*}-\left(\left\langle\Gamma_{2}\left(u_{1}^{*}, \mathcal{P} u_{n}^{*}\right)\right\rangle \cdot \nabla \lambda\right) \partial_{\theta} u_{1}^{*}=\mathcal{G}_{n-1}
$$


La symétrie de $\Gamma_{2}$ fournit :

$$
\left\langle\Gamma_{2}\left(u_{1}^{*}, \partial_{\theta} u_{1}^{*}\right)\right\rangle=\frac{1}{2}\left\langle\partial_{\theta} \Gamma_{2}\left(u_{1}^{*}, u_{1}^{*}\right)\right\rangle=0 .
$$

On en déduit :

$$
\left\langle\Gamma_{2}\left(u_{1}^{*}, \mathcal{P} u_{n}^{*}\right)\right\rangle=\left\langle\Gamma_{2}\left(u_{1}^{*}, \mathcal{P} w_{n}^{*}\right)\right\rangle .
$$

De même, par symétrie des $f_{j}^{\prime \prime}$ :

$$
\left\langle f_{j}^{\prime \prime}\left(u_{0}\right)\left(u_{1}^{*}, \mathcal{P} u_{n}^{*}\right)\right\rangle=\left\langle f_{j}^{\prime \prime}\left(u_{0}\right)\left(u_{1}^{*}, \mathcal{P} w_{n}^{*}\right)\right\rangle .
$$

On constate que les contraintes $\left(\mathcal{Z}_{n}\right)$ et (4.15) sont équivalentes au système suivant, portant sur les inconnues $\left(\mathcal{P} w_{n}^{*}, \bar{u}_{n+1}, \psi_{n}\right)$ :

$$
\left\{\begin{array}{l}
\mathcal{P}^{\prime}(X+E) \mathcal{P} w_{n}^{*}+\mathcal{P}^{\prime}\left(\left\langle\Gamma_{2}\left(u_{1}^{*}, \mathcal{P} w_{n}^{*}\right)\right\rangle \cdot \nabla \lambda\right) \partial_{\theta} u_{1}^{*}=\mathcal{G}_{n-1}, \\
M \bar{u}_{n+1}=\sum_{j=0}^{d} f_{0}^{\prime}\left(\bar{u}_{0}\right)^{-1} \partial_{j}\left\langle f_{j}^{\prime \prime}\left(\bar{u}_{0}\right)\left(\mathcal{P} u_{1}^{*}, \mathcal{P} w_{n}^{*}\right)\right\rangle+\mathcal{G}_{n-1} \\
X_{0} \psi_{n}=\bar{u}_{n+1} \cdot \nabla_{u} \lambda .
\end{array}\right.
$$

On résout ces équations dans l'ordre. La première est associée à la donnée initiale $\mathcal{P} w_{n \mid t=0}^{*}=\mathcal{P} u_{n, 0}^{*}$. Elle conduit à $w_{n}^{*}=\mathcal{P} w_{n}^{*} \in \mathcal{W}^{\infty}(T)$. Dès lors, la seconde équation met en jeu un terme source connu. Avec la donnée initiale $\bar{u}_{n+1,0}$, elle détermine $\bar{u}_{n+1} \in \mathcal{W}^{\infty}(T)$. La dernière équation donne accès à $\psi_{n}$. Enfin, le changement d'inconnues inverse de (4.16) fournit $\mathcal{P} u_{n}^{*}$. On a ainsi une solution $\left(\mathcal{P} u_{n}^{*}, \bar{u}_{n+1}\right)$ de $\left(\mathcal{Z}_{n}\right)$.

\subsection{Démonstration du théorème 3.1, cas général}

L'analyse du paragraphe 4.2 fournit une démonstration du théorème 3.1 lorsqu'on a $\bar{u}_{1}=0$. On montre maintenant que la méthode s'adapte au cas général. L'idée est que la construction des solutions BKW est explicite et dépend continûment des données $\bar{u}_{0}$ et $\varphi$.

Soit $\bar{u}_{1,0} \in H^{\infty}\left(\mathbb{R}^{d}\right)$. Pour $\delta>0$ assez petit, le problème de Cauchy pour (1.1) avec la donnée initiale

$$
\bar{u}_{0}^{\delta}(0, x)=\bar{u}_{0}(0, x)+\delta \bar{u}_{1}(0, x)
$$

admet une unique solution $\bar{u}_{0}^{\delta}(t, x)$ telle que $\bar{u}_{0}-\bar{u}_{0}^{\delta}$ est bornée dans $\mathcal{W}^{\infty}(T)$. De même, pour $\delta>0$ assez petit, l'équation eikonale

$$
\partial_{t} \varphi^{\delta}+\lambda\left(\bar{u}_{0}^{\delta}, \partial_{x} \varphi^{\delta}\right)=0, \quad \varphi^{\delta}(0, x)=\varphi_{0}(x)
$$

détermine la phase $\varphi^{\delta}$ telle que $d \varphi^{\delta}-d \varphi$ est bornée dans $\mathcal{W}^{\infty}(T)$.

On effectue la construction du paragraphe 4.2 autour des états de base $\bar{u}_{0}^{\delta}$. On obtient ainsi des solutions asymptotiques de $(\mathcal{S})$ :

$$
u^{\varepsilon, \delta}(t, x, \theta) \sim \bar{u}_{0}^{\delta}+\sum_{n \geqslant 1} \varepsilon^{n / 2} u_{n}(\delta, t, x, \theta)
$$


ou encore des solutions asymptotiques

$$
\widetilde{u}^{\varepsilon, \delta}(t, x) \sim \bar{u}_{0}^{\delta}+\sum_{n \geqslant 1} \varepsilon^{n / 2} u_{n}\left(\delta, t, x, \frac{\varphi^{\delta}(t, x)}{\varepsilon}\right)
$$

de (1.1). La construction montre que les profils $u_{n}(\delta, \cdot)$ et les phases $\varphi^{\delta}$ sont des fonctions $C^{\infty}$ en $\delta \in\left[0, \delta_{0}\right]$, si les données initiales pour les $\mathcal{P}^{\delta} u_{n}(\delta)$ et les $\bar{u}_{n+1}(\delta)$ le sont. On approche alors les différentes expressions $u_{j}^{\delta}, \varphi^{\delta}, \mathcal{P}^{\delta}, \ldots$ par leurs développements de Taylor lorsque $\delta$ tend vers zéro. En particulier :

$$
\begin{aligned}
& \bar{u}_{0}(\delta, t, x)=\bar{u}_{0}(t, x)+\delta \bar{u}_{1}(t, x)+\mathrm{O}\left(\delta^{2}\right), \\
& \varphi^{\delta}(t, x)=\varphi(t, x)+\delta \varphi^{1}(t, x)+\mathrm{O}\left(\delta^{2}\right), \\
& u_{1}(\delta, t, x)=u_{1}^{*}(t, x, \theta)+\mathrm{O}(\delta) .
\end{aligned}
$$

Les équations qui déterminent $\bar{u}_{1}$ et $\varphi^{1}$ sont respectivement le linéarisé de (1.1) en $\bar{u}_{0}$ et le linéarisé de l'équation eikonale en $\left(\varphi, \bar{u}_{0}\right)$. Ceci est conforme à ce qui est annoncé au paragraphe 2.2 .

Finalement, on peut lier les paramètres $\delta$ et $\varepsilon$ en choisissant $\delta=\sqrt{\varepsilon}$. On a alors

$$
\frac{\varphi^{\delta}}{\varepsilon}=\frac{\varphi_{\varepsilon}}{\varepsilon}+\psi(\sqrt{\varepsilon}, t, x), \quad \varphi_{\varepsilon}:=\varphi+\sqrt{\varepsilon} \varphi^{1}
$$

où $\psi$ est $C^{\infty}$ en $(\sigma, t, x)$. Développant

$$
u_{n}(\sqrt{\varepsilon}, t, x, \theta+\psi(\sqrt{\varepsilon}, t, x)) \sim \sum_{k \geqslant 0} \varepsilon^{k / 2} u_{n, k}(t, x, \theta)
$$

et regroupant les termes en $v_{n}=\sum_{p+q=n} u_{p, q}$, on voit que

$$
\widetilde{u}_{a}^{\varepsilon, \sqrt{\varepsilon}}(t, x) \sim \bar{u}_{0}+\sum_{n \geqslant 1} \varepsilon^{n / 2} v_{n}\left(t, x, \frac{\varphi_{\varepsilon}}{\varepsilon}\right)
$$

a bien la forme (1.2). En particulier, on trouve :

$$
v_{1}(t, x, \theta)=\bar{u}_{1}(t, x)+u_{1}^{*}(t, x, \theta) .
$$

Il reste à voir qu'on peut choisir arbitrairement les données initiales $\bar{v}_{n+1,0}$ et les $\mathcal{P} v_{n, 0}^{*}$ pour $n \geqslant 2$. Par exemple, on peut choisir les données initiales $\bar{u}_{n+1}(\delta)_{\mid t=0}=\bar{v}_{n+1,0}$. Dans ce cas, on a bien pour $n \geqslant 2$ :

$$
\bar{v}_{n+1 \mid t=0}=\sum_{p+q=n+1} u_{p, q \mid t=0}=\bar{v}_{n+1,0} .
$$

De même, si on choisit par récurrence sur $n$ les données initiales

$$
\mathcal{P}^{\delta} u_{n}^{*}(\delta)_{\mid t=0}=\mathcal{P}^{\delta}\left(\mathcal{P} v_{n, 0}^{*}-\sum_{p=1}^{n-1} u_{p, n-p \mid t=0}^{*}\right)
$$


on a

$$
\mathcal{P} v_{n \mid t=0}^{*}=\left(\mathcal{P}^{\delta} u_{n}^{*}(\delta)\right)_{\mid \delta=0, t=0}+\mathcal{P} \sum_{p=1}^{n-1} u_{p, n-p \mid t=0}^{*}
$$

et comme $\left(P^{\delta}\right)_{\mid \delta=0}=\mathcal{P}$, on a bien $\left(\mathcal{P} v_{n^{*}}\right)_{\mid t=0}=\mathcal{P} v_{n, 0}^{*}$.

\subsection{Interactions entre modes linéairement dégénérés}

Pour la dynamique des gaz, la dimension $\kappa$ de l'espace propre $E(u, \xi)$ est égale à $d$. Le profil $u_{1}^{*}$ se sépare en une partie de type vitesse, polarisée dans l'espace orthogonal au vecteur $\nabla \varphi$ et une composante sur l'entropie. L'analyse de stabilité indique que la composante $s$ occupe un statut à part. Il est naturel de se demander si une oscillation forte polarisée sur $s$ reste confinée à $s$, malgré les interactions qui se produisent. Si $\bar{u}_{0}$ est un état constant, on va voir que la réponse est négative mais il est intéressant de noter que pour des $\bar{u}_{0}$ généraux, les oscillations sur $s$ se transmettent effectivement à la vitesse.

Dans un premier temps, il convient d'indiquer pour un système général (1.1) les conditions d'existence de variables d'état dont le rôle est analogue à celui de l'entropie pour l'équation d'Euler. Avec les notations du paragraphe 2.1, on pose :

$$
\mathbb{F}(u):=\bigcap_{\xi \neq 0} E(u, \xi), \quad \grave{\kappa}(u):=\operatorname{dim} \mathbb{F}(u) \geqslant 0 .
$$

Pour un système général, on s'attend à ce que $\mathbb{F}(u)=\{0\}$. Toutefois, on remarque que pour le système d'Euler, $\mathbb{F}(u)$ est précisément l'espace où varie la composante associée à l'entropie. L'hypothèse suivante est satisfaite pour le système des équations d'Euler (2.37)

HYPOTHÈSE 4.1. - La dimension $\grave{\kappa}(u)$ est une constante strictement positive.

Sous cette hypothèse, le système (1.1) hérite de propriétés supplémentaires, décrites et établies dans [4]. On utilise dans ce paragraphe les renseignements suivants. D'abord il est possible de redresser $\mathbb{F}(u)$. Autrement dit, après changement de variables d'état, on peut décomposer $u=(\grave{u}, \grave{u})$ :

$$
\mathbb{F}(u)=\mathbb{F}:=\{\dot{u}=0\}=\{0\} \times \mathbb{R}^{\grave{\kappa}} .
$$

Ensuite, la valeur propre $\lambda(u, \xi)$ est linéaire en $\xi$ et indépendante de $\grave{u}$ :

$$
\lambda(u, \xi)=\xi \cdot \mu(\dot{u})=\sum_{j=1}^{d} \xi_{j} \mu_{j}(\dot{u}) .
$$

On note $\grave{\mathcal{P}}$ le projecteur (orthogonal) sur $\mathbb{F}$. On a :

$$
f_{j}^{\prime}(u) \grave{\mathcal{P}}=\mu_{j}(\dot{u}) f_{0}^{\prime}(u) \grave{\mathcal{P}}, \quad \forall u \in \mathbb{R}^{N}, \forall j \in\{1, \ldots, d\} .
$$

Après intégration, cela implique :

$$
f_{j}(u)=\mu_{j}(\dot{u}) f_{0}(u)+g_{j}(\dot{u}), \quad \forall u \in \mathbb{R}^{N}, \forall j \in\{1, \ldots, d\} .
$$

Par exemple, pour l'équation d'Euler (2.37), on a : 


$$
\begin{aligned}
& \grave{\kappa}=1, \quad \mathbb{F}=\left\{{ }^{t}(0,0, s) ; s \in \mathbb{R}\right\}, \\
& \text { pour } \quad u={ }^{t}(p, v, s): \quad \grave{u}={ }^{t}(p, v), \quad \grave{u}=s, \quad \grave{P} u={ }^{t}(0,0, s) .
\end{aligned}
$$

La décomposition (4.21) a lieu avec :

$$
f_{0}(u)=\beta(p, s)^{t}(1, v, s), \quad \mu_{j}(\dot{u})=v_{j}, \quad g_{j}(\dot{u})=p^{t}\left(\delta_{i j}\right)_{0 \leqslant i \leqslant d+1} .
$$

On cherche des solutions $\left(u_{1}^{*}, \bar{u}_{2}\right)$ de l'équation $\left(\mathcal{Z}_{1}\right)$ vérifiant la condition supplémentaire :

$$
u_{1}^{*}=\grave{\mathcal{P}} u_{1}^{*}
$$

qui implique bien sûr que $u_{1}^{*}=\mathcal{P} u_{1}^{*}$ puisque $\mathbb{F} \subset E\left(\bar{u}_{0}(t, x), \partial_{x} \varphi(t, x)\right)$. On considère donc des données initiales vérifiant :

$$
u_{1,0}^{*}=\grave{\mathcal{P}} u_{1,0}^{*} .
$$

Dans ce qui suit, on choisit pour $\mathcal{P}$ le projecteur orthogonal sur ker $\mathcal{L}_{1}$, de sorte que :

$$
\mathcal{P} \grave{\mathcal{P}}=\grave{\mathcal{P}} \mathcal{P}=\grave{\mathcal{P}}
$$

Proposition 4.3. - Les solutions de $\left(\mathcal{Z}_{1}\right)$ vérifient $(4.22)$ pour toutes les données initiales dans $H^{\infty}$ astreintes à (4.23) si et seulement si :

$$
(\mathcal{P}-\grave{\mathcal{P}}) f_{0}^{\prime}\left(\bar{u}_{0}\right)^{-1} f_{0}^{\prime \prime}\left(\bar{u}_{0}\right)\left(X_{0} \bar{u}_{0}, \grave{\mathcal{P}} v\right)=0, \quad \forall v \in \mathbb{R}^{N} .
$$

Démonstration. - On se place dans des variables où (4.18) est vérifié. On étudie la différence :

$$
d(t, x, \theta):=(\mathcal{P}-\grave{\mathcal{P}}) u_{1}^{*}(t, x, \theta)=\mathcal{P} d(t, x, \theta) .
$$

D'après (2.22), l'équation $\left(\mathcal{Z}_{1}\right)$ s'écrit encore :

$$
\mathcal{P}(X+E) \mathcal{P} u_{1}^{*}=0 .
$$

On compose à gauche avec $\grave{\mathcal{P}}$. On utilise (4.24) et le fait que la projection $\grave{\mathcal{P}}$ ne dépend pas des variables $(t, x)$ pour récupérer :

$$
\mathcal{P} X \grave{\mathcal{P}} u_{1}^{*}+\grave{\mathcal{P}} E \mathcal{P} u_{1}^{*}=0
$$

On en déduit :

$$
\mathcal{P} X \mathcal{P} d=-(\mathcal{P}-\grave{\mathcal{P}}) E \mathcal{P} d-(\mathcal{P}-\grave{\mathcal{P}}) E \grave{\mathcal{P}} u_{1}^{*}
$$

La quantité $d=\mathcal{P} d$ est donc solution d'un système différentiel. Le critère nécessaire et suffisant pour qu'une fonction $d$ nulle à l'instant initial le reste s'écrit :

$$
(\mathcal{P}-\grave{\mathcal{P}}) E \grave{\mathcal{P}} v=0, \quad \forall v \in \mathbb{R}^{N} .
$$

D'après (4.20) et la définition de la matrice $E$, on a la simplification :

$$
\begin{aligned}
(\mathcal{P}-\grave{\mathcal{P}}) E \grave{\mathcal{P}} v & =\sum_{j=0}^{d}(\mathcal{P}-\grave{\mathcal{P}}) f_{0}^{\prime}\left(\bar{u}_{0}\right)^{-1} \partial_{j}\left(\mu_{j}\left(\bar{u}_{0}\right) f_{0}^{\prime}\left(\bar{u}_{0}\right) \grave{\mathcal{P}} v\right) \\
& =(\mathcal{P}-\grave{\mathcal{P}}) f_{0}^{\prime}\left(\bar{u}_{0}\right)^{-1} f_{0}^{\prime \prime}\left(\bar{u}_{0}\right)\left(X_{0} \bar{u}_{0}, \grave{\mathcal{P}} v\right)
\end{aligned}
$$


On voit apparaître à cet endroit la condition (4.25).

En particulier, la condition (4.25) est satisfaite quand

$$
X_{0} \bar{u}_{0}=0 .
$$

Comme $\bar{u}_{0}$ est aussi solution de (1.1), on est en présence d'un système d'équations surdéterminé. Ce système admet cependant des solutions particulières. Par exemple les fonctions constantes.

Pour la dynamique des gaz (2.37), si l'état de base $\bar{u}_{0}=\left(\bar{p}_{0}, \bar{v}_{0}, \bar{s}_{0}\right)$ vérifie $X_{0}\left(\bar{p}_{0}, \bar{s}_{0}\right) \equiv 0$ et $X_{0} \bar{v}_{0} \not \equiv 0$, un simple calcul indique que la contrainte (4.26) n'est pas vérifiée. Cela signifie que des oscillations fortes polarisées à l'instant initial sur $s$ se transmettent lorsque le temps évolue en des oscillations fortes sur la vitesse. Du coup, elles engendrent une instabilité forte (cf. paragraphe 6).

\section{Stabilité hyperbolique}

On étudie la stabilité de $(\mathcal{S})$ autour d'une solution approchée $u_{a}^{\varepsilon}$ construite au paragraphe 4 . Pour simplifier, on suppose ici que $\bar{u}_{1}=0$, ce qui par l'analyse du paragraphe 4.3 n'est pas une restriction.

\subsection{Analyse du linéarisé}

On considère une solution approchée de $(\mathcal{S})$, ou plus généralement une famille de fonctions de la forme

$$
u^{\varepsilon}(t, x, \theta)=\bar{u}_{0}(t, x)+\sqrt{\varepsilon} u_{1}(t, x, \theta)+\varepsilon v^{\varepsilon}(t, x, \theta)
$$

avec $v^{\varepsilon}$ bornée dans l'espace des fonctions Lipschitziennes sur $[0, T] \times \mathbb{R}^{d} \times \mathbb{T}$. Le linéarisé de $(\mathcal{S})$ en $u^{\varepsilon}$ s'écrit :

$$
\begin{aligned}
\mathcal{L}_{c}^{\varepsilon} \dot{u}= & \sum_{j=0}^{d} \Sigma_{j}\left(u^{\varepsilon}\right) \partial_{j} \dot{u}+\frac{1}{\varepsilon} \Sigma\left(u^{\varepsilon}, \partial_{x} \varphi\right) \partial_{\theta} \dot{u} \\
& +\frac{1}{\varepsilon} \sum_{j=0}^{d} \partial_{j} \varphi\left(\dot{u} \cdot \nabla_{u} \Sigma_{j}\left(u^{\varepsilon}\right)\right) \partial_{\theta} u^{\varepsilon}+\sum_{j=0}^{d}\left(\dot{u} \cdot \nabla_{u} \Sigma_{j}\left(u^{\varepsilon}\right)\right) \partial_{j} u^{\varepsilon} .
\end{aligned}
$$

La partie différentielle est symétrique. La difficulté vient des termes singuliers. On a

$$
\Sigma\left(u^{\varepsilon}, \partial_{x} \varphi\right)=A_{0}+\sqrt{\varepsilon} A_{1}+\varepsilon A_{2}
$$

avec :

$$
\begin{aligned}
& A_{0}=S\left(\bar{u}_{0}, d \varphi\right)=\Sigma\left(\bar{u}_{0}, \partial_{x} \varphi\right), \\
& A_{1}=\left(u_{1} \cdot \nabla_{u} S\right)\left(\bar{u}_{0}, d \varphi\right)=\left(u_{1} \cdot \nabla_{u} \Sigma\right)\left(\bar{u}_{0}, \partial_{x} \varphi\right) .
\end{aligned}
$$

Ici $A_{2}$ est une fonction régulière de $\left(\varepsilon, \bar{u}_{0}, u_{1}, v, \partial_{x} \varphi\right)$. On a utilisé que $\varphi$ est solution de l'équation eikonale, que $\lambda$ est linéairement dégénérée, que $\bar{u}_{1}=0$ et que $u_{1}=u_{1}^{*}$ est polarisé sur le noyau de $\mathcal{L}_{1}$. De même :

$$
\sum_{j=0}^{d} \partial_{j} \varphi\left(\dot{u} \cdot \nabla \Sigma_{j}\left(u^{\varepsilon}\right)\right) \partial_{\theta} u^{\varepsilon}=\left(\dot{u} \cdot \nabla_{u} S\right)\left(\bar{u}_{0}, d \varphi\right) \partial_{\theta} u^{\varepsilon}=\sqrt{\varepsilon} B_{1} \dot{u}+\varepsilon B_{2} \dot{u} .
$$


Ici $B_{2}$ est une fonction régulière de $\left(\varepsilon, \bar{u}_{0}, u_{1}, v, \partial_{x} \varphi\right)$ et :

$$
B_{1} \dot{u}=\left(\dot{u} \cdot \nabla_{u} S\right)\left(\bar{u}_{0}, d \varphi\right) \partial_{\theta} u_{1} .
$$

On décompose $B_{1}$ en $B_{1}^{\prime}+B_{1}^{\prime \prime}$ avec :

$$
\left\{\begin{array}{l}
B_{1}^{\prime} \dot{u}=\dot{u} \cdot \nabla_{u} \Sigma\left(\bar{u}_{0}, \partial_{x} \varphi\right) \partial_{\theta} u_{1} \\
B_{1}^{\prime \prime} \dot{u}=\left(\dot{u} \cdot \nabla_{u} \lambda\right)\left(\bar{u}_{0}, \partial_{x} \varphi\right) \Sigma_{0}\left(\bar{u}_{0}\right) \partial_{\theta} u_{1}
\end{array}\right.
$$

Avec $R_{j}:=\Sigma_{j}\left(u^{\varepsilon}\right)$, le linéarisé s'écrit alors sous la forme

$$
\mathcal{L}_{c}^{\varepsilon}=\sum_{j=0}^{d} R_{j} \partial_{j}+A_{2} \partial_{\theta}+\frac{1}{\varepsilon} A_{0} \partial_{\theta}+\frac{1}{\sqrt{\varepsilon}}\left(A_{1} \partial_{\theta}+B_{1}\right)+C
$$

où $C$ est une fonction régulière de $\left(\varepsilon, \bar{u}_{0}, u_{1}, v, \partial_{x} \varphi\right)$.

Les $R_{j}$ et les $A_{j}$ sont des applications symétriques et uniformément Lipchitziennes. La matrice $A_{0}$ est symétrique et indépendante de $\theta$ tandis que $C$ est uniformément bornée. La méthode d'énergie standard, qui consiste à intégrer $\left(\mathcal{L}_{c}^{\varepsilon} \dot{u}, \dot{u}\right)$, conduit à un terme singulier :

$$
\frac{1}{2 \sqrt{\varepsilon}}\left(D\left(u_{1}\right) \dot{u}, \dot{u}\right), \quad D\left(u_{1}\right)=-\partial_{\theta} A_{1}+B_{1}+{ }^{t} B_{1} .
$$

On décompose cette matrice $D$ en $D^{\prime}+D^{\prime \prime}$ avec :

$$
D^{\prime}\left(u_{1}\right)=-\partial_{\theta} A_{1}+B_{1}^{\prime}+{ }^{t} B_{1}^{\prime}, \quad D^{\prime \prime}\left(u_{1}\right)=B_{1}^{\prime \prime}+{ }^{t} B_{1}^{\prime \prime} .
$$

On analyse d'abord la structure des termes singuliers. Pour des raisons évidentes de symétrie, on choisit maintenant les projecteurs $\Pi_{\perp}(u, \xi)$, projecteurs orthogonaux sur le noyau de $\Sigma(u, \xi)$. Comme au paragraphe 3 , on note $\mathcal{P}_{\perp}=\Pi_{\perp}\left(\bar{u}_{0}, \partial_{x} \varphi\right)$.

LEMME 5.1. - Les matrices $A_{1}$ et $B_{1}$ vérifient :

$$
\begin{aligned}
& \mathcal{P}_{\perp} A_{1} \mathcal{P}_{\perp}=0, \\
& \mathcal{P}_{\perp} B_{1}^{\prime}=0, \quad B_{1}^{\prime \prime} \mathcal{P}_{\perp}=0, \quad \mathcal{P}_{\perp} B_{1} \mathcal{P}_{\perp}=0, \\
& \left(B_{1}-\partial_{\theta} A_{1}\right) \mathcal{P}_{\perp}=0 .
\end{aligned}
$$

Démonstration. - C'est une conséquence des relations de transparence. On a

$$
\Sigma(u, \xi) \Pi_{\perp}(u, \xi)=0
$$

et par symétrie $\Pi_{\perp}(u, \xi) \Sigma(u, \xi)=0$. En dérivant la première identité dans la direction $h$, il vient :

$$
h \cdot \nabla_{u} \Sigma(u, \xi) \Pi_{\perp}(u, \xi)=-\Sigma(u, \xi)\left(h \cdot \nabla_{u} \Pi_{\perp}(u, \xi)\right) .
$$

En particulier :

$$
\Pi_{\perp}(u, \xi)\left(h \cdot \nabla_{u} \Sigma(u, \xi)\right) \Pi_{\perp}(u, \xi)=0 .
$$

Appliquée en $u=\bar{u}_{0}, \xi=\partial_{x} \varphi$ et $h=u_{1}$, cette identité implique que $\mathcal{P}_{\perp} A_{1} \mathcal{P}_{\perp}=0$. Comme $\partial_{\theta} u_{1}=\Pi_{\perp}\left(\bar{u}_{0}, \xi\right) \partial_{\theta} u_{1}$, on a aussi $\mathcal{P}_{\perp} B_{1}^{\prime}=0$. Par ailleurs, $h \cdot \nabla \lambda(u, \xi)=0$ huand

$$
h=\Pi_{\perp}(u, \xi) h,
$$

d'où $B_{1}^{\prime \prime} \mathcal{P}_{\perp}=0$. 
On pose $h_{1}=\partial_{\theta} u_{1}=\Pi_{\perp} h_{1}$. On se donne $k$ avec $k=\Pi_{\perp} k$. On a :

$$
\left(B_{1}-\partial_{\theta} A_{1}\right) k=\left(k \cdot \nabla_{u} \Sigma\right) h_{1}-\left(h_{1} \cdot \nabla_{u} \Sigma\right) k,
$$

les matrices étant évaluées en $u=\bar{u}_{0}$ et $\xi=\partial_{x} \varphi$. On a aussi via (5.12) :

$$
\left(B_{1}-\partial_{\theta} A_{1}\right) k=-\Sigma\left(\left(k \cdot \nabla_{u} \Pi_{\perp}\right) h_{1}-\left(h_{1} \cdot \nabla_{u} \Pi_{\perp}\right) k\right) .
$$

On interprète cette égalité à l'aide de (4.5). On trouve :

$$
\left(B_{1}-\partial_{\theta} A_{1}\right) k=-\Sigma\left(\Gamma_{2}\left(k, h_{1}\right)-\Gamma_{2}\left(h_{1}, k\right)\right) .
$$

La symétrie de $\Gamma_{2}$ donne $\left(B_{1}-\partial_{\theta} A_{1}\right) \mathcal{P}_{\perp}=0$.

On peut visualiser ce lemme sous une forme matricielle. Dans une base constituée d'une base de $\operatorname{ker} \mathcal{P}_{\perp}$ et d'une base de im $\mathcal{P}_{\perp}$, on a :

$$
\begin{aligned}
& A_{0}=\left(\begin{array}{cc}
A_{0}^{1,1} & 0 \\
0 & 0
\end{array}\right), \quad A_{1}=\left(\begin{array}{cc}
A_{1}^{1,1} & A_{1}^{1,2} \\
{ }^{t} A_{1}^{1,2} & 0
\end{array}\right), \\
& B_{1}^{\prime}=\left(\begin{array}{cc}
B_{1}^{\prime 1,1} & \partial_{\theta} A_{1}^{1,2} \\
0 & 0
\end{array}\right), \quad B_{1}^{\prime \prime}=\left(\begin{array}{cc}
B_{1}^{\prime \prime 1,1} & 0 \\
B_{1}^{\prime \prime 2,1} & 0
\end{array}\right) .
\end{aligned}
$$

Par conséquent, la matrice $D$ définie en (5.7) se met sous la forme :

$$
D=\left(\begin{array}{cc}
B_{1}^{1,1}+{ }^{t} B_{1}^{1,1}-\partial_{\theta} A_{1}^{1,1} & { }^{t} B_{1}^{\prime \prime 2,1} \\
B_{1}^{\prime \prime 2,1} & 0
\end{array}\right) .
$$

On peut simplifier la forme du linéarisé à l'aide d'un changement d'inconnues

$$
\dot{u}=\phi^{\varepsilon} \check{u}, \quad \phi^{\varepsilon}=\mathrm{Id}+\sqrt{\varepsilon} \phi_{1}(t, x, \theta),
$$

où $\phi_{1}$ est une matrice régulière en $(t, x, \theta)$. On pose alors :

$$
\check{\mathcal{L}} \check{u}:={ }^{t} \phi^{\varepsilon} \mathcal{L}_{c}^{\varepsilon} \dot{u}={ }^{t} \phi^{\varepsilon} \mathcal{L}_{c}^{\varepsilon} \phi^{\varepsilon} \check{u} .
$$

L'opérateur $\check{\mathcal{L}}$ a clairement la même forme que $\mathcal{L}_{c}^{\varepsilon}$ :

$$
\check{\mathcal{L}}=\sum_{j=0}^{d} \check{R}_{j} \partial_{j}+\check{A}_{2} \partial_{\theta}+\frac{1}{\varepsilon} \check{A}_{0} \partial_{\theta}+\frac{1}{\sqrt{\varepsilon}}\left(\check{A}_{1} \partial_{\theta}+\check{B}_{1}\right)+\check{C} .
$$

De nouveau les matrices symétriques $\check{R}_{j}$ et $\check{A}_{j}$ sont des fonctions régulières de $\left(u_{0}, u_{1}, v\right)$ et $\phi_{1}$. La partie $\breve{C}$ dépend en outre des dérivées de $\phi_{1}$. On a :

$$
\left\{\begin{array}{l}
\check{A}_{0}=A_{0} \\
\check{A}_{1}=A_{1}+{ }^{t} \phi_{1} A_{0}+A_{0} \phi_{1} \\
\check{B}_{1}=B_{1}+A_{0} \partial_{\theta} \phi_{1} .
\end{array}\right.
$$

Proposition 5.1.- On peut choisir $\phi_{1}$ de sorte que :

$$
\begin{aligned}
& \check{A}_{1}=0, \quad \check{B}_{1} \mathcal{P}_{\perp}=0, \\
& \mathcal{P}_{\perp} \check{B}_{1}\left(\mathrm{Id}-\mathcal{P}_{\perp}\right)=\mathcal{P}_{\perp} B_{1}^{\prime \prime}\left(\mathrm{Id}-\mathcal{P}_{\perp}\right), \\
& \left(\mathrm{Id}-\mathcal{P}_{\perp}\right) \check{B}_{1}\left(\mathrm{Id}-\mathcal{P}_{\perp}\right)=\frac{1}{2}\left(\operatorname{Id}-\mathcal{P}_{\perp}\right)\left(B_{1}+{ }^{t} B_{1}-\partial_{\theta} A_{1}\right)\left(\mathrm{Id}-\mathcal{P}_{\perp}\right)
\end{aligned}
$$


Démonstration. - On note $\mathcal{Q}_{\perp}=Q_{\perp}\left(\bar{u}_{0}, \partial_{x} \varphi\right)$ où $Q_{\perp}(u, \xi)$ désigne l'inverse partiel de $\Sigma(u, \xi)$. Avec ces conventions, on a :

$$
Q_{\perp} \Sigma=\Sigma Q_{\perp}=\mathrm{Id}-\Pi_{\perp}, \quad Q_{\perp} \Pi_{\perp}=\Pi_{\perp} Q_{\perp}=0, \quad A_{0} \mathcal{Q}_{\perp}=\mathrm{Id}-\mathcal{P}_{\perp} .
$$

On pose :

$$
\phi_{1}:=-\mathcal{Q}_{\perp}\left(\frac{1}{2} A_{1}\left(\mathrm{Id}-\mathcal{P}_{\perp}\right)+A_{1} \mathcal{P}_{\perp}+Z\left(\mathrm{Id}-\mathcal{P}_{\perp}\right)\right) .
$$

La matrice $Z$ est pour l'instant inconnue. On prend $Z$ antisymétrique. Ainsi l'introduction de $Z$ ne modifie pas le calcul de $\check{A}_{1}$ qui livre, pour ce choix de $\phi_{1}$, le premier résultat $\check{A}_{1}=0$.

On se tourne alors vers $\check{B}_{1}$. On obtient :

$$
\check{B}_{1}=B_{1}-\frac{1}{2}\left(\mathrm{Id}-\mathcal{P}_{\perp}\right) \partial_{\theta} A_{1}\left(\mathrm{Id}-\mathcal{P}_{\perp}\right)-\left(\operatorname{Id}-\mathcal{P}_{\perp}\right) \partial_{\theta} A_{1} \mathcal{P}_{\perp}-\left(\operatorname{Id}-\mathcal{P}_{\perp}\right) \partial_{\theta} Z\left(\operatorname{Id}-\mathcal{P}_{\perp}\right) .
$$

En particulier :

$$
\check{B}_{1} \mathcal{P}_{\perp}=B_{1} \mathcal{P}_{\perp}-\left(\mathrm{Id}-\mathcal{P}_{\perp}\right) \partial_{\theta} A_{1} \mathcal{P}_{\perp} .
$$

Les relations établies au lemme 5.1 fournissent :

$$
\check{B}_{1} \mathcal{P}_{\perp}=0, \quad \mathcal{P}_{\perp} \check{B}_{1}\left(\mathrm{Id}-\mathcal{P}_{\perp}\right)=\mathcal{P}_{\perp} B_{1}^{\prime \prime}\left(\mathrm{Id}-\mathcal{P}_{\perp}\right) .
$$

Il reste à traiter :

$$
\left(\mathrm{Id}-\mathcal{P}_{\perp}\right) \check{B}_{1}\left(\mathrm{Id}-\mathcal{P}_{\perp}\right)=\left(\mathrm{Id}-\mathcal{P}_{\perp}\right)\left(B_{1}-\frac{1}{2} \partial_{\theta} A_{1}-\partial_{\theta} Z\right)\left(\mathrm{Id}-\mathcal{P}_{\perp}\right)
$$

On rappelle que $B_{1}$ est une fonction linéaire de $u_{1}=u_{1}^{*}$. C'est donc une fonction de moyenne nulle en $\theta$. Le terme $\partial_{\theta} Z$ permet d'absorber la partie antisymétrique de $B_{1}$. Il suffit pour cela de poser $\partial_{\theta} Z=\frac{1}{2}\left(B_{1}-{ }^{t} B_{1}\right)$. Dès lors, on voit apparaître la dernière égalité de la proposition 5.1 .

Dans une base comme en (5.14), on a :

$$
\check{B}_{1}=\left(\begin{array}{cc}
\check{B}_{1}^{1,1} & 0 \\
B_{1}^{\prime \prime 2,1} & 0
\end{array}\right) \quad \text { avec } \check{B}_{1}^{1,1}={ }^{t} \check{B}_{1}^{1,1}
$$

Comme $A_{0}$ est symétrique et ne dépend que des variables $(t, x)$, on a, comme on pouvait le prévoir :

$$
\check{D}:=-\partial_{\theta} \check{A}_{1}+\check{B}_{1}+{ }^{t} \check{B}_{1}=\check{B}_{1}+{ }^{t} \check{B}_{1}=D
$$

Soit encore :

$$
\check{D}=D=\left(\begin{array}{cc}
2 \check{B}_{1}^{1,1} & { }^{t} B_{1}^{\prime \prime 2,1} \\
B_{1}^{\prime \prime 2,1} & 0
\end{array}\right) .
$$

On termine ce paragraphe par un examen du terme :

$$
D^{\prime}\left(u_{1}\right)=-\partial_{\theta} A_{1}+B_{1}^{\prime}+{ }^{t} B_{1}^{\prime} .
$$

On se sert des définitions (2.25), (5.4) et (5.6) pour exprimer le produit scalaire $\left(D^{\prime} \dot{u}, \dot{u}\right)$ autrement :

$$
\left(D^{\prime} \dot{u}, \dot{u}\right)=2 B l^{\prime}\left(\bar{u}_{0}, \partial_{x} \varphi\right)\left(\partial_{\theta} u_{1} ; \dot{u}, \dot{u}\right) .
$$

On peut à présent prouver que la condition $D^{\prime}\left(u_{1}\right)=0$ a une signification intrinsèque. 
LEMME 5.2. - Considérant $\Sigma(u, \xi)$ comme un tenseur 2 fois covariant, pour tout $(u, \xi) \in$ $\mathcal{U} \times \mathbb{R}^{d} \backslash\{0\}$ et tout $h_{1} \in E(u, \xi), B l^{\prime}(u, \xi)\left(h_{1} ; \cdot, \cdot\right)$ est la dérivée de Lie de ce tenseur en $(u, \xi)$, le long d'un champ $X(v)$ défini au voisinage de $u$, tel que $X(\cdot) \in E(\cdot, \xi)$ et $X(u)=h_{1}$.

Démonstration. - On fixe $\xi \in \mathbb{R}^{d} \backslash\{0\}$. Soit $X$ un champ tel que :

$$
X(u)=h_{1}, \quad(\operatorname{Id}-\Pi)(v, \xi) X(v)=0, \quad \forall v \in \mathcal{U} .
$$

Autrement dit, il existe une fonction $h(v)$ vérifiant :

$$
h(u)=h_{1}, \quad X(v)=\Pi(v, \xi) h(v), \quad \forall v \in \mathcal{U} .
$$

La dérivée de $X$ en $u$ le long du vecteur $k$ est donnée par :

$$
X^{\prime}(u) k=\left(k \cdot \nabla_{u} \Pi\right)(u, \xi) h_{1}+\Pi(u, \xi)(k \cdot \nabla h(u)) .
$$

La quantité $X^{\prime}(u)$ peut être représentée comme une matrice de taille $N \times N$. D'après (5.12), on a :

$$
\left(k \cdot \nabla_{u} \Sigma\right)(u, \xi) h_{1}=-\Sigma(u, \xi)\left(k \cdot \nabla_{u} \Pi\right)(u, \xi) h_{1}=-\Sigma(u, \xi) X^{\prime}(u) k .
$$

La matrice de la forme quadratique associée à $B l^{\prime}\left(h_{1} ; \cdot, \cdot\right)$ s'interprète alors, au regard de ce qui précède et de la définition (2.25), suivant :

$$
\left(X \cdot \nabla_{u} \Sigma+\Sigma X^{\prime}+{ }^{t} X^{\prime} \Sigma\right)(u, \xi)
$$

On reconnaît en cette expression la dérivée de Lie du tenseur 2 fois covariant $\Sigma(\cdot, \xi)$ le long du champ $X$.

\subsection{Bon symétriseur}

La notion de bon symétriseur est dégagée dans [13,24,6]. Elle concerne la dimension un d'espace. Elle s'applique par exemple au système de lois de conservation :

$$
\partial_{t} f_{0}(u)+\partial_{y}\left(\sum_{j=1}^{d} \xi_{j} f_{j}(u)\right)=0 .
$$

Le vecteur $\xi \in \mathbb{R}^{d} \backslash\{0\}$ est fixé. La lettre $y$ répresente la variable $y=\xi \cdot x \in \mathbb{R}$. La notion de bon symétriseur s'exprime dans des variables d'état $u$ où le feuilletage $\Im_{\xi}$ est redressé comme indiqué en (2.4). L'espace propre $E(u, \xi)$ est alors indépendant de $u$. Notons le $E(\xi)$. Dans ces conditions, on dit que $\Sigma_{0}$ est un bon symétriseur pour (5.22) lorsque :

$$
\left(h \cdot \nabla_{u}\right) \Sigma(u, \xi)=0, \quad \forall(u, h) \in \mathcal{U} \times E(\xi) .
$$

Ceci revient à dire que si l'on écrit $u=(z, w)$ comme en $(2.4), \Sigma(u, \xi)$ est indépendant des variables $w$.

Preuve de la proposition 3.1. - Par changement de variables $u=\Phi(v)$, la matrice $\Sigma(u, \xi)$ se transforme en $\widetilde{\Sigma}(v, \xi)={ }^{t} \Phi^{\prime}(v) \Sigma(u, \xi) \Phi^{\prime}(v)$, c'est-à-dire comme un tenseur deux fois covariant ( $\Sigma$ est une forme quadratique sur l'espace tangent à $\mathcal{U}$ ). Dans les coordonnées redressées, la dérivée de Lie le long du champ constant $h$ tangent au feuilletage $\Im_{\xi}$ coüncide avec la dérivée classique $h \cdot \nabla_{u}$. La condition (5.23) exprime que ces dérivées sont nulles. La notion de dérivée de Lie est intrinsèque, préservée par changement de variables. Par conséquent, (5.23) 
est équivalent à l'annulation de la dérivée de Lie de $\Sigma(\cdot, \xi)$ le long des champs tangents aux feuilles de $\Im_{\xi}$.

D'après le lemme 5.2, cette condition est équivalente à l'annulation de $B l^{\prime}\left(h_{1} ; \cdot, \cdot\right)$ pour tous $u \in \mathcal{U}$ et $h_{1} \in E(u, \xi)$.

Pour un système général, le changement de variables $\phi_{\xi}$ qui redresse le feuilletage $\Im_{\xi}$ dépend effectivement de la direction $\xi \in \mathbb{S}^{d-1}$. Il arrive néanmoins, par exemple dans le cas de la dynamique des gaz, que l'on puisse redresser les $\Im_{\xi}$ simultanément en $\xi$.

DÉFINITION 5.1. - On dit que la famille de feuilletages $\left\{\Im_{\xi}\right\}_{\xi \in \mathbb{S}^{d-1}}$ possède une rigidité affine si pour tout $\xi$ dans $\mathbb{S}^{d-1}$, les feuilles de $\Im_{\xi}$ sont des sous-espaces affines de dimension $\kappa$, parallèles.

Il est possible de caractériser la rigidité affine autrement :

LEMME 5.3. - Les affirmations suivantes sont équivalentes:

(i) La famille $\left\{\Im_{\xi}\right\}_{\xi \in \mathbb{S}^{d-1}}$ possède une rigidité affine.

(ii) Pour tout $\xi$ dans $\mathbb{S}^{d-1}$, la projection orthogonale $\Pi_{\perp}(u, \xi)$ ne dépend pas de la variable $u$.

(iii) On $a$ :

$$
\left(v \cdot \nabla_{u} \Sigma\right)(u, \xi) \Pi_{\perp}(u, \xi) w=0, \quad \forall(v, w, u, \xi) .
$$

Démonstration. - Par définition, (i) équivaut à dire que pour tout $\xi \in \mathbb{S}^{d-1}$, il existe un espace $E(\xi)$ de dimension $\kappa$ tel que la feuille de $\Im_{\xi}$ passant par $u$ est $(u+E(\xi)) \cap \mathcal{U}$, ou encore que $E(u, \xi)=E(\xi)$ pour tout $u \in \mathcal{U}$. Cela revient à dire que $\Pi_{\perp}$ est indépendant de $u$.

D'après (5.12), l'idendité (5.24) s'écrit aussi :

$$
\Sigma\left(v \cdot \nabla_{u} \Pi_{\perp}\right) \Pi_{\perp} \equiv 0 .
$$

De plus, en dérivant l'identité $\Pi_{\perp} \circ \Pi_{\perp}=\Pi_{\perp}$, on obtient :

$$
\left(v \cdot \nabla_{u} \Pi_{\perp}\right) \Pi_{\perp}=\left(\mathrm{Id}-\Pi_{\perp}\right)\left(v \cdot \nabla_{u} \Pi_{\perp}\right) .
$$

Comme $\Sigma$ est inversible sur l'image de Id $-\Pi_{\perp}$, (5.24) équivaut à :

$$
\left(v \cdot \nabla_{u} \Pi_{\perp}\right) \Pi_{\perp}=\left(\mathrm{Id}-\Pi_{\perp}\right)\left(v \cdot \nabla_{u} \Pi_{\perp}\right) \equiv 0 .
$$

Compte tenu de la symétrie de la matrice $v \cdot \nabla_{u} \Pi_{\perp}$, cette condition revient à imposer $v \cdot \nabla_{u} \Pi_{\perp}=0$. On retrouve la condition (ii).

En présence de rigidité affine, il se produit de nombreuses simplifications. Par exemple, la contribution $\Gamma_{2}$ disparaît puisqu'on a :

$$
\Gamma_{2}\left(w_{1}, w_{2}\right)=\left(\operatorname{Id}-\Pi_{\perp}\right)\left(w_{1} \cdot \nabla_{u} \Pi_{\perp}\right) w_{2}=0 .
$$

Par ailleurs, $\Sigma_{0}$ est un bon symétriseur si et seulement si :

$$
\Sigma(u, \xi)=\Sigma\left(\left(\operatorname{Id}-\Pi_{\perp}\right)(\xi) u, \xi\right), \quad \forall(u, \xi) \in \mathcal{U} \times \mathbb{S}^{d-1} .
$$

Exemple 5.1.- On termine ce paragraphe en donnant des exemples de systèmes quasilinéaires qui possèdent de la rigidité affine et un bon symétriseur. On se donne $d$ scalaires $\left\{\mu_{j}(u)\right\}_{1 \leqslant j \leqslant d}$ et $d$ matrices $\left\{M_{j}\right\}_{1 \leqslant j \leqslant d}$ symétriques et constantes. On suppose que le noyau de l'application linéaire $M(\xi)=\sum_{j=1}^{d} \xi_{j} M_{j}$ est de dimension $\kappa>0$ constante lorsque $\xi$ parcourt $\mathbb{S}^{d-1}$. Par exemple :

$$
M(\xi)={ }^{t} R\left(\begin{array}{cc}
0 & { }^{t} \xi \\
\xi & 0
\end{array}\right) R .
$$


Soit une matrice $\Sigma_{0}(u)$ définie positive. On considère alors le système défini par les matrices :

$$
\Sigma_{j}(u):=\mu_{j}(u) \Sigma_{0}(u)+M_{j}, \quad 1 \leqslant j \leqslant d .
$$

La valeur propre

$$
\lambda(u, \xi)=\sum \xi_{j} \mu_{j}(u)
$$

est de multiplicité constante et le noyau $E(u, \xi)=\operatorname{ker} M(\xi)$ est indépendant de $u$. De plus $\Sigma(u, \xi)=M(\xi)$ et $\Sigma_{0}$ est un bon symétriseur. Pour que $\lambda$ soit linéairement dégénérée il faut et il suffit que $v \cdot \nabla_{u} \mu(u) \in \xi^{\perp}$ pour $v \in \operatorname{ker} M(\xi)$. Si les $\mu_{j}$ sont constants, alors $\lambda$ est linéairement dégénérée et vérifie la condition (3.6). Le système ainsi construit est conservatif si $\Sigma_{0}=\eta^{\prime \prime}$ est la matrice Hessienne d'une fonction $\eta$ strictement convexe. On notera que le système construit est effectivement non linéaire.

\subsection{Preuve du théorème 3.2}

Soit :

$$
u_{a}^{\varepsilon}=\bar{u}_{0}+\sqrt{\varepsilon} u_{1}+\varepsilon v_{a}^{\varepsilon}
$$

une solution approchée dans $\mathcal{O}_{a}^{s}(r)$ avec $r>s+1>\frac{d+5}{2}$. En particulier, $v_{a}^{\varepsilon}$ est borné dans $\mathcal{W}^{s+1}(T)$. On suppose en outre que $\bar{u}_{1}=0$ et que la matrice $D$ définie en (5.7) est nulle. On se donne enfin une famille $R^{\varepsilon}$ bornée dans $\mathcal{W}_{\varepsilon}^{s}(T)$ et une famille $I^{\varepsilon}$ bornée dans $H^{s}\left(\mathbb{R}^{d} \times \mathbb{T}\right)$. Il s'agit de montrer que, pour $\varepsilon$ assez petit, le problème de Cauchy

$$
\mathcal{S}\left(u^{\varepsilon} ; \partial_{t, x, \theta}\right) u^{\varepsilon}=R_{a}^{\varepsilon}+\varepsilon^{r} R^{\varepsilon}, \quad u^{\varepsilon}(0, x, \theta)=u_{a}^{\varepsilon}(0, x, \theta)+\varepsilon^{r} I^{\varepsilon}
$$

admet une solution dans $\mathcal{W}^{s}(T)$ et qu'il existe $C$ tel que pour $\left.\left.\varepsilon \in\right] 0, \varepsilon_{0}\right]$ :

$$
\left\|u^{\varepsilon}-u_{a}^{\varepsilon}\right\|_{\mathcal{W}_{\varepsilon}^{s}(T)} \leqslant C \varepsilon^{r} .
$$

On cherche la solution sous la forme $u^{\varepsilon}=u_{a}^{\varepsilon}+\varepsilon^{r} d^{\varepsilon}$. L'équation pour $d^{\varepsilon}$ s'écrit :

$$
\mathcal{S}\left(u^{\varepsilon}, \partial_{t, x, \theta}\right) d^{\varepsilon}+\mathcal{S}^{\prime}\left(t, x, \varepsilon^{r} d^{\varepsilon}\right) d^{\varepsilon}=\varepsilon^{-r} R_{a}^{\varepsilon}+R^{\varepsilon},\left.\quad d^{\varepsilon}\right|_{t=0}=I^{\varepsilon}
$$

avec :

$$
\begin{aligned}
\mathcal{S}^{\prime}(t, x, v) h:= & \sum_{j=0}^{d} \int_{0}^{1}\left(h \cdot \nabla_{u} \Sigma_{j}\right)\left(u_{a}^{\varepsilon}(t, x)+s v\right) \partial_{j} u_{a}^{\varepsilon}(t, x) d s \\
& +\frac{1}{\varepsilon} \sum_{j=0}^{d} \partial_{j} \varphi(t, x) \int_{0}^{1}\left(h \cdot \nabla_{u} \Sigma_{j}\right)\left(u_{a}^{\varepsilon}(t, x)+s v\right) \partial_{\theta} u_{a}^{\varepsilon}(t, x) d s .
\end{aligned}
$$

Le système (5.28) est hyperbolique symétrisable à données $H^{s}$. Il possède une solution locale en temps (voir [19]), de durée de vie $T^{*}(\varepsilon)>0$. De plus, si $T^{*}(\varepsilon)<+\infty$, on a nécessairement :

$$
\limsup _{t \rightarrow T^{*}(\varepsilon)-}\left\|u^{\varepsilon}(t, \cdot)\right\|_{H^{s}\left(\mathbb{R}^{d} \times \mathbb{T}\right)}=+\infty .
$$

En particulier :

$$
\lim _{t \rightarrow T^{*}(\varepsilon)-}\left\|u^{\varepsilon}\right\|_{\mathcal{W}_{\varepsilon}^{s}(t)}=+\infty
$$


Le théorème 3.2 résulte alors de :

$$
\begin{aligned}
& \exists \varepsilon_{0}>0 ; \quad \inf _{\left.\varepsilon \in] 0, \varepsilon_{0}\right]} T^{*}(\varepsilon)>T, \\
& \sup _{\left.\varepsilon \in] 0, \varepsilon_{0}\right]}\left\|d^{\varepsilon}\right\|_{\mathcal{W}_{\varepsilon}^{s}(T)}<+\infty .
\end{aligned}
$$

Compte tenu des critères d'explosion rappelés ci-dessus, il suffit de montrer qu'il existe des constantes $\varepsilon_{0}$ et $M$ telles que :

$$
\left.\forall \varepsilon \in] 0, \varepsilon_{0}\right], \forall T^{\prime} \leqslant \min \left(T^{*}(\varepsilon), T\right), \quad\left\|d^{\varepsilon}\right\|_{\mathcal{W}_{\varepsilon}^{s}\left(T^{\prime}\right)} \leqslant M
$$

La démonstration se fait en deux temps. D'abord, on utilise la proposition 5.1 pour effectuer un changement d'inconnues qui transforme l'équation (5.28) en une équation similaire dont les termes singuliers sont à coefficients constants. Ensuite, on applique une méthode de commutateurs pour estimer les normes $H^{s}$ de la solution de (5.28).

Réduction de l'équation. On écrit :

$$
\Sigma\left(u^{\varepsilon}, \partial_{x} \varphi\right)=\Sigma\left(\bar{u}_{0}, \partial_{x} \varphi\right)+\sqrt{\varepsilon}\left(u_{1} \cdot \nabla_{u} \Sigma\right)\left(\bar{u}_{0}, \partial_{x} \varphi\right)+\varepsilon \mathcal{R}_{d+1}\left(\varepsilon, t, x, v_{a}^{\varepsilon}+\varepsilon^{r-1} d^{\varepsilon}\right)
$$

où $R_{d+1}$ est une matrice régulière de ses arguments. De même

$$
\mathcal{S}^{\prime}\left(t, x, \varepsilon^{r} d^{\varepsilon}\right)=\frac{1}{\sqrt{\varepsilon}} B_{1}+E\left(\varepsilon, t, x, v_{a}^{\varepsilon}, \partial_{t, x, \theta} v_{a}^{\varepsilon}, \varepsilon^{r-1} d^{\varepsilon}\right)
$$

où $B_{1}$ est défini en (5.6) et $E$ est une fonction $C^{\infty}$ de ses arguments. L'équation pour $d^{\varepsilon}$ est donc de la forme

$$
\begin{gathered}
\mathcal{R}\left(\varepsilon, v_{a}^{\varepsilon}, \varepsilon^{r-1} d^{\varepsilon}, \partial_{t, x, \theta}\right) d^{\varepsilon}+E\left(\varepsilon, t, x, v_{a}^{\varepsilon}, \partial_{t, x, \theta} v_{a}^{\varepsilon}, \varepsilon^{r-1} d^{\varepsilon}\right) d^{\varepsilon} \\
+\frac{1}{\varepsilon} A_{0} \partial_{\theta} d^{\varepsilon}+\frac{1}{\sqrt{\varepsilon}}\left(A_{1} \partial_{\theta} d^{\varepsilon}+B_{1} d^{\varepsilon}\right)=\varepsilon^{-r} R_{a}^{\varepsilon}+R^{\varepsilon}
\end{gathered}
$$

où les coefficients de l'opérateur quasi-linéaire symétrique $\mathcal{R}$ sont des fonctions régulières de $\left(\varepsilon, v_{a}^{\varepsilon}, \varepsilon^{r-1} d^{\varepsilon}\right)$.

Ensuite, on applique le changement d'inconnues $d^{\varepsilon}=\left(\operatorname{Id}+\sqrt{\varepsilon} \phi_{1}\right) \breve{d}^{\varepsilon}$ avec $\phi_{1}$ donné à la proposition 5.1. Comme $D=0$, la formule (5.20) implique que $\breve{d}^{\varepsilon}$ vérifie :

$$
\check{\mathcal{R}}\left(\varepsilon, v_{a}^{\varepsilon}, \varepsilon^{r-1} \check{d}^{\varepsilon}, \partial_{t, x, \theta}\right) \check{d}^{\varepsilon}+\check{E}\left(\varepsilon, t, x, v_{a}^{\varepsilon}, \partial_{t, x, \theta} v_{a}^{\varepsilon}, \varepsilon^{r-1} \check{d}^{\varepsilon}\right) \check{d}^{\varepsilon}+\frac{1}{\varepsilon} A_{0} \partial_{\theta} \check{d}^{\varepsilon}=\check{R}^{\varepsilon} .
$$

Ici, on a posé :

$$
\check{R}^{\varepsilon}:=\left(\operatorname{Id}+\sqrt{\varepsilon}^{t} \phi_{1}\right)\left(\varepsilon^{-r} R_{a}^{\varepsilon}+R^{\varepsilon}\right) .
$$

Par ailleurs $\check{\mathcal{R}}$ est de même nature que $\mathcal{R}$.

La matrice $A_{0}=\Sigma\left(\bar{u}_{0}, \partial_{x} \varphi\right)$ dépend des seules variables $(t, x)$. D'après l'hypothèse 2.1 , elle est de rang constant. Cela implique l'existence d'une matrice inversible régulière $\check{\phi}(t, x)$ telle que $\underline{\Sigma}:={ }^{t} \breve{\phi}(t, x) \Sigma\left(\bar{u}_{0}, \partial_{x} \varphi\right) \check{\phi}(t, x)$ est constante. On pose $\widetilde{d}^{\varepsilon}:=\check{\phi}(t, x)^{-1} \breve{d}^{\varepsilon}$ de sorte que $\widetilde{d}^{\varepsilon}$ est solution de :

$$
\begin{aligned}
& \widetilde{\mathcal{R}}\left(\varepsilon, v_{a}^{\varepsilon}, \varepsilon^{r-1} \widetilde{d}^{\varepsilon}, \partial_{t, x, \theta}\right) \widetilde{d}^{\varepsilon}+\widetilde{E}\left(\varepsilon, t, x, v_{a}^{\varepsilon}, \partial_{t, x, \theta} v_{a}^{\varepsilon}, \varepsilon^{r-1} \widetilde{d}^{\varepsilon}\right) \widetilde{d}^{\varepsilon} \\
& \quad+\frac{1}{\varepsilon} \underline{\Sigma} \partial_{\theta} \widetilde{d}^{\varepsilon}=\widetilde{R}^{\varepsilon}:=\check{\phi} \check{R}^{\varepsilon}
\end{aligned}
$$

où $\widetilde{\mathcal{R}}$ et $\widetilde{E}$ sont de même nature que $\mathcal{R}$ et $E$. 
Il suffit maintenant de résoudre le problème de Cauchy pour (5.32) avec pour nouvelles données initiales :

$$
\widetilde{d}_{\mid t=0}^{\varepsilon}=\widetilde{I}^{\varepsilon}:=\check{\phi}_{\mid t=0}^{-1}\left(\operatorname{Id}+\sqrt{\varepsilon} \phi_{1 \mid t=0}\right)^{-1} I^{\varepsilon} .
$$

Le contrôle annoncé en (5.31) résulte alors de l'estimation :

$$
\left.\forall \varepsilon \in] 0, \varepsilon_{0}\right], \forall T^{\prime}<\min \left(T^{*}(\varepsilon), T\right), \quad\left\|\widetilde{d}^{\varepsilon}\right\|_{\mathcal{W}_{\varepsilon}^{s}\left(T^{\prime}\right)} \leqslant M .
$$

Estimations d'énergie. On raisonne dorénavant sur (5.32). La démonstration des majorations (5.34) est inspirée du travail de O. Guès [11] (voir aussi G. Browning et H.O. Kreiss [3]).

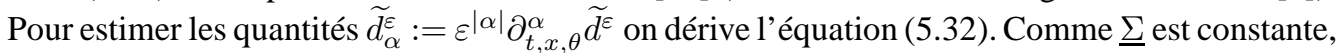
on trouve que

$$
\widetilde{\mathcal{R}}\left(\varepsilon, v_{a}^{\varepsilon}, \varepsilon^{r-1} \widetilde{d}^{\varepsilon}, \partial_{t, x, \theta}\right) \widetilde{d}_{\alpha}^{\varepsilon}+\frac{1}{\varepsilon} \underline{\Sigma} \widetilde{d}_{\alpha}^{\varepsilon}=\widetilde{R}_{\alpha}^{\varepsilon}
$$

où :

$$
\widetilde{R}_{\alpha}^{\varepsilon}=\varepsilon^{|\alpha|} \partial^{\alpha}\left(\widetilde{R}^{\varepsilon}-\widetilde{E}\left(\varepsilon^{r-1} \widetilde{d}^{\varepsilon}\right) \widetilde{d}^{\varepsilon}\right)-\left[\varepsilon^{|\alpha|} \partial^{\alpha}, \mathcal{R}\left(\varepsilon^{r-1} \widetilde{d}^{\varepsilon}, \partial_{t, x, \theta}\right)\right] \widetilde{d}^{\varepsilon}
$$

Dans ces expressions, on a simplifié l'écriture de $\widetilde{\mathcal{R}}$ et $\widetilde{E}$ pour ne retenir que la dépendance en $\widetilde{d}^{\varepsilon}$. Les autres coefficients $v_{a}^{\varepsilon}$ ne sont pas mentionnés. Leur intervention ne pose pas de problème car ils sont bornés dans $\mathcal{W}^{s+1}(T)$. On évalue le terme de droite. Par hypothèse, le terme $\varepsilon^{|\alpha|} \partial^{\alpha} \widetilde{R}^{\varepsilon}$ est borné dans $C^{0}\left([0, T] ; L^{2}\right)$ puisque $\widetilde{R}^{\varepsilon}$ se déduit de $R^{\varepsilon}$ par multiplication par une matrice $C^{\infty}$ bornée dans $\mathcal{W}^{\infty}(T)$. Les autre termes sont sommes d'expressions de la forme

$$
h=\varepsilon^{|\alpha|+(r-1)(p-1)} \Phi \partial^{\alpha^{1}} \widetilde{d}^{\varepsilon} \ldots \partial^{\alpha^{p}} \widetilde{d}^{\varepsilon} \partial^{\beta^{1}} v_{a}^{\varepsilon} \ldots \partial^{\beta^{q}} v_{a}^{\varepsilon}
$$

où $\Phi$ est une fonction $C^{\infty}$ des arguments $\left(t, x, v_{a}^{\varepsilon}, \varepsilon^{r-1} \widetilde{d}^{\varepsilon}\right)$, et les multiindices $\alpha^{j}$ et $\beta^{k}$ vérifient :

$$
\begin{aligned}
& l:=\left|\alpha^{1}\right|+\cdots+\left|\alpha^{p}\right| \leqslant|\alpha|, \\
& l^{\prime}:=\left|\beta^{1}\right|+\cdots+\left|\beta^{q}\right| \leqslant|\alpha|+1, \\
& l+l^{\prime} \leqslant|\alpha|+1 .
\end{aligned}
$$

En utilisant les règles de multiplication dans les espaces de Sobolev

$$
H^{s^{\prime}}\left(\mathbb{R}^{d} \times \mathbb{T}\right) \times H^{s^{\prime \prime}}\left(\mathbb{R}^{d} \times \mathbb{T}\right) \subset H^{s^{\prime \prime \prime}}\left(\mathbb{R}^{d} \times \mathbb{T}\right)
$$

quand $0 \leqslant s^{\prime \prime \prime} \leqslant \min \left(s^{\prime}, s^{\prime \prime}\right)$ et $s^{\prime}+s^{\prime \prime}-s^{\prime \prime \prime}>\frac{d+1}{2}$, on voit que pour $s>\frac{d+3}{2},|\alpha| \leqslant s$ et $t \leqslant T^{\prime}<\min \left(T^{*}(\varepsilon), T\right)$, on récupère :

$$
\begin{aligned}
\|h(t)\|_{L^{2}\left(\mathbb{R}^{d} \times \mathbb{T}\right)} \leqslant & C \varepsilon^{|\alpha|+(r-1)(p-1)}\|\Phi\|_{L^{\infty}\left(\left[0, T^{\prime}\right] \times \mathbb{R}^{d} \times \mathbb{T}\right)}\left\|v_{a}^{\varepsilon}\right\|_{\mathcal{W}^{s+1}\left(T^{\prime}\right)}^{q} \\
& \times\left\|\widetilde{d}^{\varepsilon}\right\|_{\mathcal{W}^{s}\left(T^{\prime}\right)}^{p-1}\left\|\widetilde{d}^{\varepsilon}\right\|_{\mathcal{W}^{|\alpha|}\left(T^{\prime}\right)} .
\end{aligned}
$$

Comme $v_{a}^{\varepsilon}$ est bornée dans $\mathcal{W}^{s+1}$ et que $\delta=r-1-s>0$, on voit que pour $|\alpha| \leqslant s$, on a :

$$
\|h(t)\|_{L^{2}} \leqslant C\|\Phi\|_{L^{\infty}}\left(1+\varepsilon^{\delta}\left\|\widetilde{d}^{\varepsilon}\right\|_{\mathcal{W}_{\varepsilon}^{s}\left(T^{\prime}\right)}\right)^{p-1}\left\|\widetilde{d}^{\varepsilon}\right\|_{\mathcal{W}_{\varepsilon}^{s}\left(T^{\prime}\right)} .
$$

L'injection de Sobolev implique que pour $s \geqslant 1+\frac{d+1}{2}$ :

$$
\|w\|_{L^{\infty}}+\|\partial w\|_{L^{\infty}} \leqslant C \varepsilon^{-1-\frac{d+1}{2}}\|w\|_{\mathcal{W}_{\varepsilon}^{s}\left(T^{\prime}\right)} .
$$


Comme $v_{a}^{\varepsilon}$ est bornée, cela permet de majorer $\Phi$ dans $L^{\infty}$. On voit alors qu'il existe une fonction continue $K(\cdot)$ telle que pour $|\alpha| \leqslant s$ on a pour $t \in\left[0, T^{\prime}\right]$ et $T^{\prime}<\min \left(T^{*}(\varepsilon), T\right)$ :

$$
\left\|\widetilde{R}_{\alpha}^{\varepsilon}(t)\right\|_{L^{2}\left(\mathbb{R}^{d} \times \mathbb{T}\right)} \leqslant K\left(\varepsilon^{\delta} M^{\varepsilon}\left(T^{\prime}\right)\right) M^{\varepsilon}\left(T^{\prime}\right)
$$

avec $M^{\varepsilon}\left(T^{\prime}\right):=\left\|\widetilde{d^{\varepsilon}}\right\|_{\mathcal{W}_{\varepsilon}^{s}\left(T^{\prime}\right)}$.

D'autre part, (5.36) implique que les coefficients de $\widetilde{\mathcal{R}}$ sont bornés et ont des dérivées premières bornées par $K_{1}\left(\varepsilon^{\delta} M^{\varepsilon}\left(T^{\prime}\right)\right)$. Il en va de même pour l'inverse du coefficient de $\partial_{t}$. Comme $\widetilde{\mathcal{R}}$ et $\underline{\Sigma}$ sont symétriques, une intégration par parties montre qu'il existe des fonctions $K_{1}(\cdot)$ et $K_{2}(\cdot)$, telles que pour $t \in\left[0, T^{\prime}\right]$ et $T^{\prime}<\min \left(T^{*}(\varepsilon), T\right)$ :

$$
\begin{aligned}
\left\|\widetilde{d}_{\alpha}^{\varepsilon}(t)\right\|_{L^{2}\left(\mathbb{R}^{d} \times \mathbb{T}\right)} \leqslant & K_{1}(M) e^{t K_{2}(M)}\left\|\widetilde{d}_{\alpha}^{\varepsilon}(0)\right\|_{L^{2}\left(\mathbb{R}^{d} \times \mathbb{T}\right)} \\
& +K_{1}(M) \int_{0}^{t} e^{\left(t-t^{\prime}\right) K_{2}(M)}\left\|\widetilde{R}_{\alpha}^{\varepsilon}\left(t^{\prime}\right)\right\|_{L^{2}\left(\mathbb{R}^{d} \times \mathbb{T}\right)} d t^{\prime}
\end{aligned}
$$

avec $M=M^{\varepsilon}\left(T^{\prime}\right)$.

On estime maintenant les données initiales $\widetilde{d}_{\alpha}^{\varepsilon}(0)$. Pour cela on utilise l'équation (5.32) qui permet d'exprimer les dérivées temporelles $\partial_{t}^{j} \widetilde{d}^{\varepsilon}(t, \cdot)$ en fonction des dérivées spatiales $\partial_{x, \theta}^{\alpha} \widetilde{d}^{\varepsilon}(t, \cdot)$ de longueur $|\alpha| \leqslant j$. La présence du terme singulier $\frac{1}{\varepsilon} \Sigma \partial_{\theta}$ fait en sorte que chaque dérivation en temps consomme une puissance du paramètre $\varepsilon$. Cette perte est compensée par le recours à une norme à poids, en l'occurrence celle de l'espace $\mathcal{W}_{\varepsilon}^{s}$. Par un calcul analogue à celui utilisé pour les commutateurs, on obtient qu'il existe une constante $C_{0}$, qui ne dépend que des normes de $u_{0}, u_{1}$, d'un majorant des normes de $v_{a}^{\varepsilon}$ dans $\mathcal{W}^{s+1}(T)$ et de $I^{\varepsilon}$ dans $H^{s}\left(\mathbb{R}^{d} \times \mathbb{T}\right)$ telle que pour tout $\varepsilon \in] 0,1]$, on a :

$$
\left\|\varepsilon^{j} \partial_{t}^{j} \widetilde{d}^{\varepsilon}(0, \cdot)\right\|_{H^{s-j}\left(\mathbb{R}^{d} \times \mathbb{T}\right)} \leqslant C_{0} .
$$

En particulier, les normes $L^{2}$ des $\widetilde{d}_{\alpha}^{\varepsilon}(0)$ sont uniformément bornées.

En sommant (5.38) pour tous $|\alpha| \leqslant s$ et en utilisant (5.37) et le lemme de Gronwall, on en déduit qu'il existe des fonctions $K_{1}$ et $K_{2}$ telles que pour tout $T^{\prime}<\min \left(T^{*}(\varepsilon), T\right)$ on a :

$$
\left\|\widetilde{d}^{\varepsilon}\right\|_{\mathcal{W}_{\varepsilon}^{s}\left(T^{\prime}\right)} \leqslant K_{1}\left(\varepsilon^{\delta} M^{\varepsilon}\left(T^{\prime}\right)\right) e^{T^{\prime} K_{2}\left(\varepsilon^{\delta} M^{\varepsilon}\left(T^{\prime}\right)\right)} .
$$

Fin de la démonstration du théorème 3.2. On choisit $M>\max \left(C_{0}, K_{1}(1) e^{T K_{2}(1)}\right)$ puis $\varepsilon_{0}$ tel que $\varepsilon_{0}^{\delta} M \leqslant 1$. Alors, par un argument usuel de continuation, (5.39) implique que pour $\varepsilon \leqslant \varepsilon_{0}$ et $T^{\prime}<\min \left(T^{*}(\varepsilon), T\right)$ on a $M^{\varepsilon}\left(T^{\prime}\right) \leqslant M$. L'estimation (5.34) en résulte et la démonstration du théorème 3.2 est complète.

\section{Instabilités}

Les oscillations à l'échelle $\varepsilon$ portées par le profil principal $u_{1}$ interagissent avec les oscillations de plus petites amplitudes portées par la perturbation $\dot{u}$. Ces interactions sont susceptibles d'induire des mécanismes d'amplification qui, éventuellement, se traduisent, au niveau des équations de modulation fournies par une analyse BKW à deux phases, par des taux de croissance en temps plus ou moins forts. Réciproquement ces taux de croissance indiquent quels types d'estimations d'énergie sont possibles pour le linéarisé $\mathcal{L}_{c}^{\varepsilon}$. Dans un premier temps, on formalise cette idée. Ensuite on se tourne vers le problème non linéaire. On met à jour des conditions 
suffisantes pour l'existence d'instabilités non linéaires, dont un exemple typique est celui des instabilités de Rayleigh (cf. [9]).

\subsection{Divers types d'instabilité}

On se place dans le cadre du paragraphe 3.3. On en reprend les notations. On commence par établir :

THÉORÈME 6.1. - Si la famille $\mathcal{L}_{c}^{\varepsilon}$ est stable dans $L^{2}$ de type $k$, alors on a pour tout $\mathbf{v} \in C^{1}\left(\left[0, \infty\left[; H^{1}\left(\mathbb{R}^{d} \times \mathbb{T}^{2}\right)\right)\right.\right.$ et tout $s \geqslant 0$ :

$$
\|\mathbb{P} \mathbf{v}(s)\|_{L^{2}} \leqslant k(s)\left(\|\mathbb{P} \mathbf{v}(0)\|_{L^{2}}+\int_{0}^{s}\left\|\mathbb{L} \mathbf{v}\left(s^{\prime}\right)\right\|_{L^{2}} d s^{\prime}\right) .
$$

Démonstration. - On commence par établir (6.1) lorsque v est un polynôme trigonométrique en les variables $\theta$ et $\omega$, à coefficients très réguliers :

$$
\mathbf{v}(s, x, \theta, \omega)=\sum_{\text {finie }} \mathbf{v}_{\alpha, \beta}(s, x) e^{i(\alpha \theta+\beta \omega)}, \quad \mathbf{v}_{\alpha, \beta} \in C^{1}\left([0, T] ; H^{\infty}\left(\mathbb{R}^{d}\right)\right) .
$$

On pose :

$$
\dot{v}(t, x, \theta)=\mathbf{v}\left(\frac{t}{\sqrt{\varepsilon}}, x, \theta, \frac{\psi}{\varepsilon}\right) .
$$

On fait agir $\mathcal{L}_{c}^{\varepsilon}$ sur la fonction test $\dot{v}$. Il sort :

$$
\mathcal{L}_{c}^{\varepsilon} \dot{v}=\frac{1}{\varepsilon} S\left(\underline{u}, d \psi \partial_{\omega}+d \varphi \partial_{\theta}\right) \mathbf{v}+\frac{1}{\sqrt{\varepsilon}}\left(\underline{\Sigma}_{0} \partial_{s}+G\right) \mathbf{v}+\mathbf{f}
$$

où $\mathbf{f}=\mathrm{O}\left(\partial_{s, x, \theta, \omega} \mathbf{v}\right)+\mathrm{O}(\mathbf{v})$. On sépare $\mathbf{v}$ en $\mathbf{v}=\mathbf{v}_{0}+\sqrt{\varepsilon} \mathbf{v}_{1}$ où $\mathbf{v}_{0}$ est un polynôme trigonométrique à coefficients réguliers, sélectionné dans le noyau de la projection $\operatorname{Id}-\mathbb{P}$. Ainsi :

$$
S\left(\underline{u}, d \psi \partial_{\omega}+d \varphi \partial_{\theta}\right) \mathbf{v}_{0}=0 .
$$

On note $Q_{\alpha, \beta}$ l'inverse de $\underline{S}(\alpha d \varphi+\beta d \psi)$ lorsque $(\alpha, \beta) \notin Z$ ou un inverse partiel lorsque $(\alpha, \beta) \in Z$. Les $Q_{\alpha, \beta}$ servent à définir un opérateur $\mathbb{Q}$ qui agit sur les polynômes trigonométriques conformément à :

$$
\mathbb{Q}\left(\sum_{\text {finie }} \mathbf{v}_{\alpha, \beta} e^{i(\alpha \theta+\beta \omega)}\right)=\sum_{\text {finie }} Q_{\alpha, \beta} \mathbf{v}_{\alpha, \beta} e^{i(\alpha \theta+\beta \omega)} .
$$

En particulier, on repère le polynôme trigonométrique

$$
\mathbf{v}_{1}=-\mathbb{Q}\left(\underline{\Sigma}_{0} \partial_{s}+G\right) \mathbf{v}_{0}
$$

qui est ajusté de façon à ce que :

$$
S\left(\underline{u}, d \psi \partial_{\omega}+d \varphi \partial_{\theta}\right) \mathbf{v}_{1}=-(\operatorname{Id}-\mathbb{P})\left(\underline{\Sigma}_{0} \partial_{s}+G\right) \mathbf{v}_{0} .
$$

Avec ces choix, on a la simplification :

$$
\mathcal{L}_{c}^{\varepsilon} \dot{v}=\frac{1}{\sqrt{\varepsilon}} \mathbb{L} \mathbf{v}_{0}+\mathbf{f}_{1}
$$

$4^{\mathrm{e}}$ SÉRIE - TOME $36-2003-\mathrm{N}^{\circ} 5$ 
où $\mathbf{f}_{1}=\mathrm{O}\left(\partial_{s, x, \theta, \omega}\left(\mathbf{v}_{0}, \mathbf{v}_{1}\right)\right)+\mathrm{O}\left(\mathbf{v}_{0}, \mathbf{v}_{1}\right)$. On reporte $\dot{v}$ au niveau de (3.18). On remplace $t$ par $\sqrt{\varepsilon} s$ puis on effectue le changement de variables $t^{\prime}=\sqrt{\varepsilon} s^{\prime}$ dans l'intégrale. Il sort

$$
\begin{array}{r}
\left\|\mathbf{v}_{0}(s)+\sqrt{\varepsilon} \mathbf{v}_{1}(s)\right\|_{L^{2}} \leqslant k(s)\left(\left\|\mathbf{v}_{0}(0)+\sqrt{\varepsilon} \mathbf{v}_{1}(0)\right\|_{L^{2}}\right. \\
\left.+\int_{0}^{s}\left\|\mathbb{L} \mathbf{v}_{0}\left(s^{\prime}\right)+\sqrt{\varepsilon} \mathbf{f}_{1}\left(s^{\prime}\right)\right\|_{L^{2}} d s^{\prime}\right)
\end{array}
$$

où il faut prendre garde à ce que les normes $L^{2}$ sont évaluées en $(x, \theta)$, la phase non stationnaire $\psi$ étant substituée à la variable $\omega$. On fait tendre $\varepsilon$ vers zéro. Il reste :

$$
\left\|\mathbf{v}_{0}(s)\right\|_{L^{2}} \leqslant k(s)\left(\left\|\mathbf{v}_{0}(0)\right\|_{L^{2}}+\int_{0}^{s}\left\|\mathbb{L} \mathbf{v}_{0}\left(s^{\prime}\right)\right\|_{L^{2}} d s^{\prime}\right)
$$

où cette fois-ci les normes $L^{2}$ sont en $(x, \theta, \omega)$. L'opérateur $\mathbb{L}$ agit continûment de $C^{1}([0, \infty[$; $\left.H^{1}\left(\mathbb{R}^{d} \times \mathbb{T}^{2}\right)\right)$ dans $C^{0}\left(\left[0, \infty\left[; L^{2}\left(\mathbb{R}^{d} \times \mathbb{T}^{2}\right)\right)\right.\right.$. Par densité des polynômes trigonométriques, on récupère (6.1) pour toute fonction test $\mathbf{v}$ ayant la régularité indiquée.

Preuve du théorème 3.3. - Soit $\mathbf{v}$ dans $H^{\infty}\left(\mathbb{R}^{d} \times \mathbb{T}^{2}\right)$. Comme le semi-groupe $e^{-s \mathbb{G}}$ préserve la régularité, la fonction $e^{-s \mathbb{G}} \mathbf{v}$ est, entre autres, dans l'espace $C^{1}\left(\left[0, \infty\left[; H^{1}\left(\mathbb{R}^{d} \times \mathbb{T}^{2}\right)\right)\right.\right.$. On applique (6.1) avec pour fonction test $e^{-s \mathbb{G}} \mathbf{V}$ ce qui donne :

$$
\left\|e^{-s \mathbb{G}} \mathbf{v}\right\|_{L^{2}} \leqslant k(s)\|\mathbf{v}\|_{L^{2}}, \quad \forall s \in \mathbb{R}^{+} .
$$

Par continuité de $e^{-s \mathbb{G}}$ sur $L^{2}$, cela implique :

$$
g(s):=|| e^{-s \mathbb{G}} \mid \| \leqslant k(s), \quad \forall s \in \mathbb{R}^{+} .
$$

La fonction $g$ est bien contrôlée par $k$.

Le théorème 3.3 est utile dans la pratique, comme en témoigne la discussion du paragraphe 3.3. Par orthogonalité, on a :

$$
g_{\beta} \preceq g \preceq \sup _{\beta \in \mathbb{Z}} g_{\beta} .
$$

Le comportement de la fonction $g$ est le reflet de celui des $g_{\beta}$. Les propriétés de $g_{\beta}$ sont déterminées par l'action de $\mathbb{G}_{\beta}$. C'est pourquoi il est intéressant de dégager la structure de $\mathbb{G}_{\beta}$.

Considérons un profil $u_{1}^{*}$ qui possède seulement deux harmoniques de signes opposés $+\delta$ et $-\delta$ :

$$
u_{1}^{*}(t, \theta)=r e^{i \delta \theta}+\bar{r} e^{-i \delta \theta}, \quad \delta \in \mathbb{N} .
$$

Ici, $r$ est un vecteur fixé dans $E(\underline{u}, \underline{\xi})$ et $\bar{r}$ désigne son complexe conjugué. La fonction $u_{1}^{*}$ est bien à valeurs réelles. De plus ce choix de $u_{1}^{*}$ est compatible avec la forme des équations $\left(\mathcal{Z}_{1}\right)$ du paragraphe 4.2. On note $\mathbb{G}_{\beta}^{\delta}$ l'opérateur $\mathbb{G}_{\beta}$ associé à un tel $u_{1}^{*}$. Tout $\mathbb{G}_{\beta}$ s'obtient par combinaison linéaire des $\mathbb{G}_{\beta}^{\delta}$ où $\delta$ parcourt $\mathbb{N}$. Pour simplifier les calculs, on fixe $(\beta, \delta)$ et on concentre notre attention sur $\mathbb{G}_{\beta}^{\delta}$.

L'opérateur $\mathbb{G}_{\beta}^{\delta}$ est un opérateur différentiel du premier ordre que l'on écrit sous la forme $e^{i \delta \theta} G^{+}\left(\partial_{\theta}\right)+e^{-i \delta \theta} G^{-}\left(\partial_{\theta}\right)$ avec :

$$
\left\{\begin{array}{l}
G^{+}\left(\partial_{\theta}\right) v=r \cdot \nabla_{u} S\left(\underline{u}, i \beta d \psi+d \varphi \partial_{\theta}\right) v+i \delta v \cdot \nabla_{u} S(\underline{u}, d \varphi) r \\
G^{-}\left(\partial_{\theta}\right) v=\bar{r} \cdot \nabla_{u} S\left(\underline{u}, i \beta d \psi+d \varphi \partial_{\theta}\right) v-i \delta v \cdot \nabla_{u} S(\underline{u}, d \varphi) \bar{r} .
\end{array}\right.
$$


On regarde comment $\mathbb{G}_{\beta}^{\delta}$ agit sur les modes de Fourier. On trouve :

$$
\mathbb{G}_{\beta}^{\delta}\left(\sum v_{\alpha} e^{i \alpha \theta}\right)=\sum\left(G_{\alpha}^{+} v_{\alpha-\delta}+G_{\alpha}^{-} v_{\alpha+\delta}\right) e^{i \alpha \theta}
$$

avec :

$$
G_{\alpha}^{+}:=P_{\alpha, \beta} G^{+}(i(\alpha-\delta)) P_{\alpha-\delta, \beta}, \quad G_{\alpha}^{-}:=P_{\alpha, \beta} G^{-}(i(\alpha+\delta)) P_{\alpha+\delta, \beta} .
$$

L'opérateur $\mathbb{G}_{\beta}^{\delta}$ s'interprète ainsi comme un système matriciel de dimension infinie. C'est l'analyse spectrale de ce système qui renseigne sur le comportement de $g_{\beta}$. Il arrive que cette analyse se simplifie.

Exemple 6.1. - On reprend la situation examinée en 3.1.b) dans le cas isentropique, avec $\gamma:=-2 \beta(\xi \cdot \underline{\xi}) /|\underline{\xi}|^{2}$ dans $\mathbb{Z}^{*}$. On prend $\underline{\xi}={ }^{t}(1,0)$ et $r={ }^{t}(0,1)$. On écrit :

$$
\underline{\Sigma}_{0}=\operatorname{diag}\left(\sigma_{1}, \sigma_{2}, \sigma_{2}\right), \quad \sigma_{1} \sigma_{2} \underline{c}^{2}=1, \quad \sigma_{1}>0, \quad \sigma_{2}>0 .
$$

On considère deux phases caractéristiques. L'une est associée au mode l.d.g. tandis que l'autre correspond à un mode acoustique :

$$
\varphi(t, x)=\underline{\xi} \cdot x, \quad \psi(t, x)=-\underline{c}|\xi| t+\xi \cdot x .
$$

Ces deux phases engendrent une troisième phase caractéristique :

$$
\phi(t, x)=\gamma \varphi(t, x)+\beta \psi(t, x)=\beta\left(-\underline{c}|\xi| t-\xi_{1} x_{1}+\xi_{2} x_{2}\right) .
$$

Les noyaux des matrices $\underline{S}(d \psi)$ et $\underline{S}(d \phi)$ sont de dimension un, engendrés respectivement par les vecteurs :

$$
r_{1}={ }^{t}\left(|\xi| / \sigma_{1} \underline{c}, \xi_{1}, \xi_{2}\right), \quad r_{2}={ }^{t}\left(|\xi| / \sigma_{1} \underline{c},-\xi_{1}, \xi_{2}\right) .
$$

La projection $P_{\alpha, \beta}$ est non nulle seulement si $\alpha=0$ ou $\alpha=\gamma$. Par conséquent $\mathbb{G}_{\beta}^{\delta}$ est non trivial seulement si $\delta=-\gamma$ ou $\delta=\gamma$. On examine le cas $\delta=-\gamma$, l'autre cas étant analogue. L'action de $\mathbb{G}_{\beta}^{-\gamma}$ se réduit à :

$$
\mathbb{G}_{\beta}^{-\gamma}\left(\sum v_{\alpha} e^{i \alpha \theta}\right)=G_{0}^{+} v_{\gamma}+G_{\gamma}^{-} v_{0} e^{i \gamma \theta}
$$

L'équation $\mathbb{L}_{\beta} \check{u}=0$ se formule dans la base engendrée par les vecteurs $r_{1}$ et $r_{2}$. Elle est équivalente au système différentiel de dimension deux :

$$
\partial_{s} \check{u}+\left(\begin{array}{cc}
0 & c_{+} \\
c_{-} & 0
\end{array}\right) \check{u}=0, \quad \check{u}=\left(\begin{array}{c}
\check{u}_{1} \\
\check{u}_{2}
\end{array}\right) \in \mathbb{R}^{2} .
$$

Les scalaires $c_{+}$et $c_{-}$sont identifiés via :

$$
c_{+}={ }^{t} r_{1} G^{+}(i \gamma) r_{2}, \quad c_{-}={ }^{t} r_{2} G^{-}(0) r_{1} .
$$

Pour Euler, on trouve :

$$
G^{ \pm}(i \gamma) v=i \beta(r \cdot \xi) \underline{\Sigma}_{0} v \pm i \delta(v \cdot \underline{\xi}) \underline{\Sigma}_{0} r .
$$

On en déduit les relations :

$$
c_{+}=c_{-}=i \beta(r \cdot \xi)^{t} r_{1} \underline{\Sigma}_{0} r_{2}+i \gamma \sigma_{2} \xi_{1} \xi_{2} .
$$


Il s'ensuit que :

$$
\exists c_{1}>0 ; \quad g_{\beta} \preceq c_{1}, \quad \forall \beta \in \mathbb{Z} .
$$

Autrement dit, les interactions entre modes l.d.g. et modes acoustiques ne provoquent pas, du point de vue de l'optique géométrique, des phénomènes d'amplification.

On a vu que par transparence $\mathbb{G}_{0}=0$. Pour $\beta \neq 0$ fixé, l'opérateur $\mathbb{G}_{\beta}$ est en général non trivial. L'action de $\mathbb{G}_{\beta}$ met en jeu les $\mathbb{G}_{\alpha}^{ \pm}$qui, pour les grandes valeurs de $\alpha$, ressemblent aux $\mathbb{G}_{\alpha}^{ \pm}$associés à $\mathbb{G}_{0}$. Sous cet angle $\mathbb{G}_{\beta}$ peut être perçu comme une perturbation de $\mathbb{G}_{0}$. Ce point de vue conduit au résultat suivant :

Lemme 6.1. - Soient $\beta \in \mathbb{Z}$ et $k \in \mathbb{N}$ fixés. L'opérateur $\mathbb{G}_{\beta}$ est borné de l'espace $H^{k}(\mathbb{T})$ dans lui-même.

Démonstration. - Il suffit de prouver l'estimation :

$$
\sup _{\alpha \in \mathbb{Z}} \max \left(\left\|G_{\alpha}^{+}\right\| ;\left\|G_{\alpha}^{-}\right\|\right)<+\infty .
$$

On raisonne sur les $G_{\alpha}^{+}$. Les $G_{\alpha}^{-}$se traitent de manière analogue. L'opérateur $G_{\alpha}^{+}$est non trivial seulement si les deux couples $(\alpha, \beta)$ et $(\alpha-\delta, \beta)$ donnent lieu à des phases caractéristiques. Pour $\alpha \neq 0$, on a alors :

$$
\begin{aligned}
& \operatorname{ker} S(\underline{u}, \alpha d \varphi+\beta d \psi)=\operatorname{ker} S(\underline{u}, d \varphi+\beta d \psi / \alpha) \neq\{0\}, \\
& \operatorname{ker} S(\underline{u},(\alpha-\delta) d \varphi+\beta d \psi)=\operatorname{ker} S(\underline{u},(1-\delta / \alpha) d \varphi+\beta d \psi / \alpha) \neq\{0\} .
\end{aligned}
$$

Cela implique :

$$
\underline{\tau}+\beta \tau / \alpha=-\lambda(\underline{u}, \underline{\xi}+\beta \xi / \alpha), \quad(1-\delta / \alpha) \underline{\tau}+\beta \tau / \alpha=-\lambda(\underline{u},(1-\delta / \alpha) \underline{\xi}+\beta \xi / \alpha) .
$$

On a aussi :

$$
P_{\alpha, \beta}=\Pi(\underline{u}, \underline{\xi}+\beta \xi / \alpha), \quad P_{\alpha-\delta, \beta}=\Pi(\underline{u},(1-\delta / \alpha) \underline{\xi}+\beta \xi / \alpha) .
$$

On isole le terme principal du développement asymptotique en $\alpha$ de $G_{\alpha}^{+}$lorsque $\alpha$ tend vers $\pm \infty$. On obtient

$$
G_{\alpha}^{+}=i \alpha \underline{\Pi}_{\perp}\left[r \cdot \nabla_{u} S(\underline{u},(\underline{\tau}, \underline{\xi}))\right] \underline{\Pi}_{\perp}+R_{\alpha}, \quad \underline{\Pi}_{\perp}:=\Pi_{\perp}(\underline{u}, \underline{\xi})
$$

où les restes $R_{\alpha}$ satisfont la majoration :

$$
\sup _{\alpha \in \mathbb{Z}}\left\|R_{\alpha}\right\|<+\infty
$$

Le vecteur $r$ est polarisé dans $E(\underline{u}, \underline{\xi})$. Avec l'hypothèse 2.2, on a la simplification :

$$
\underline{\Pi}_{\perp}\left[r \cdot \nabla_{u} S(\underline{u},(\underline{\tau}, \underline{\xi}))\right] \underline{\Pi}_{\perp}=\underline{\Pi}_{\perp}\left[r \cdot \nabla_{u} \Sigma(\underline{u}, \underline{\xi})\right] \underline{\Pi}_{\perp} .
$$

On se souvient alors de la relation (5.13), obtenue comme conséquence des conditions de transparence, qui garantit l'élimination de cette dernière quantité. Bref, on a $G_{\alpha}^{+}=R_{\alpha}$. Le lemme 6.1 suit.

Le lemme 6.1 est un résultat de type perturbatif. Rien ne garantit que les normes des opérateurs $\mathbb{G}_{\beta}$ soient uniformément bornées lorsque $\beta$ parcourt $\mathbb{Z}$. 
On déduit du lemme 6.1 que, mesurant les normes dans l'espace des opérateurs bornés de $L^{2}(\mathbb{T})$ dans lui-même (ou de $H^{k}(\mathbb{T})$ dans lui-même), on a :

$$
g_{\beta}(s) \leqslant e^{s|| \mathbb{G}_{\beta}|| \mid}, \quad \forall s \in \mathbb{R}^{+} .
$$

On voit ici que le semi-groupe $e^{-s \mathbb{G}_{\beta}}$ présente un taux de croissance au plus exponentielle. On peut arriver à la même conclusion en utilisant le caractère symétrique de la partie principale de $G_{\beta}$. Cela étant dit, le résultat du lemme 6.1 est notablement plus fort.

\subsection{Instabilités non linéaires}

Les instabilités linéaires mises à jour dans l'étude de l'exemple 3.4 conduisent à des amplifications exponentielles. On s'attend à ce que ces amplifications se traduisent en instabilités pour le problème non linéaire lui-même, dans l'esprit des résultats d'E. Grenier [10]. Inspirés par la dynamique des gaz, nous développons cette idée dans le cadre suivant. On se donne $\underline{u}$, et on suppose que la valeur propre $\lambda$ est telle que $\lambda(\underline{u}, \xi)$ est linéaire en $\xi$ :

$$
\lambda(\underline{u}, \xi)=\underline{a} \cdot \xi, \quad \underline{a} \in \mathbb{R}^{d} .
$$

On se donne la phase $\varphi=\underline{\tau} t+\underline{\xi} \cdot x$ avec $\underline{\xi} \neq 0$ et $\underline{\tau}=-\underline{a} \cdot \underline{\xi}$. On considère une solution approchée $u_{a}^{\varepsilon} \in \mathcal{O}_{a}^{\infty}(\infty)$ d'ordre infini avec $\bar{u}_{0}=\underline{u}$ et $u_{1}$ de la forme (6.2) avec $\delta=1$. On se ramène à $\underline{\Sigma}_{0}=$ Id. On remarque que la solution approchée $u_{a}^{\varepsilon}$ construite au paragraphe 4 existe et est régulière pour tout temps, donc en particulier sur $[0,1] \times \mathbb{R}^{d} \times \mathbb{T}$.

Pour $\xi$ linéairement indépendant de $\xi$, on considère une seconde phase, elle aussi associée à $\lambda$, notée $\psi=\tau t+\xi \cdot x$ avec $\tau=-\underline{a} \cdot \xi$. Les non linéarités conduisent à considérer non seulement la phase $\psi$ mais aussi ses harmoniques. On définit donc l'ensemble $Z$ comme en (3.12). La linéarité de $\lambda$ en $\xi$ implique que $Z$ coïncide avec $\mathbb{Z}^{2}$.

Rappel des notations. On note $\underline{S}=L(\underline{u}, \cdot)$ et $\nabla \underline{S}=\left(\nabla_{u} S\right)(\underline{u}, \cdot)$. Pour $(\alpha, \beta) \in \mathbb{Z}^{2}$, $P_{\alpha, \beta}$ désigne le projecteur orthogonal sur ker $\underline{S}(\alpha d \varphi+\beta d \psi)$. Par décomposition en séries de Fourier, ces projecteurs induisent dans $L^{2}\left(\mathbb{T}^{2} ; \mathbb{C}^{N}\right)$ un projecteur orthogonal $\mathbb{P}$ sur le noyau de $\underline{S}\left(d \varphi \partial_{\theta}+d \psi \partial_{\omega}\right)$. De même, pour chaque $\beta \in \mathbb{Z}$, on note $\mathbb{P}_{\beta}$ le projecteur orthogonal dans $L^{2}\left(\mathbb{T} ; \mathbb{C}^{N}\right)$ sur le noyau de $\underline{S}\left(i \beta d \psi+d \varphi \partial_{\theta}\right)$ et

$$
G_{\beta}\left(\theta, \partial_{\theta}\right)=e^{i \theta} G_{\beta}^{+}\left(\partial_{\theta}\right)+e^{-i \theta} G_{\beta}^{-}\left(\partial_{\theta}\right)
$$

avec :

$$
\left\{\begin{array}{l}
G_{\beta}^{+}\left(\partial_{\theta}\right) v=r \cdot \nabla \underline{S}\left(i \beta d \psi+d \varphi \partial_{\theta}\right) v+i \beta v \cdot \nabla \underline{S}(d \varphi) r, \\
G_{\beta}^{-}\left(\partial_{\theta}\right) v=\bar{r} \cdot \nabla \underline{S}\left(i \beta d \psi+d \varphi \partial_{\theta}\right) v-i \beta v \cdot \nabla \underline{S}(d \varphi) \bar{r} .
\end{array}\right.
$$

On désigne par $\mathbb{G}_{\beta}$ l'opérateur $\mathbb{P}_{\beta} G_{\beta} \mathbb{P}_{\beta}$.

HYPOTHÈSE 6.1.-

(i) L'opérateur $\mathbb{G}_{1}$ admet une valeur propre $\mu$ telle que $\gamma_{0}:=-\Re e \mu>0$, associée à une fonction propre $w \in H^{\infty}(\mathbb{T})$.

(ii) Pour tout $\gamma>\gamma_{0}$ il existe $C$ tel que :

$$
g(s) \leqslant C e^{\gamma s}, \quad \forall s \in \mathbb{R}^{+} .
$$

(iii) Les inverses partiels $Q_{\alpha, \beta}$ de $\underline{S}(\alpha d \varphi+\beta d \psi)$ sont uniformément bornés pour $(\alpha, \beta)$ parcourant $\mathbb{Z}^{2}$. 
L'hypothèse 6.1 est motivée par l'étude des équations d'Euler. L'énoncé suivant reprend celui du théorème 3.4 .

THÉORÈME 6.2. - Sous l'hypothèse 6.1, pour tous $m \in \mathbb{N}$ et $\delta>0$, il existe des constantes $C$, $\varepsilon_{0}>0$ et $c>0$, et une famille $\left\{u^{\varepsilon}\right\}_{\left.\varepsilon \in] 0, \varepsilon_{0}\right]}$ de solutions du système $(\mathcal{S})$, l'expression $u^{\varepsilon}$ étant définie sur l'intervalle $[0, T(\varepsilon)]$, telle que pour tout $\left.\varepsilon \in] 0, \varepsilon_{0}\right]$ on a :

$$
\begin{aligned}
& \left\|u_{a}^{\varepsilon}(0)-u^{\varepsilon}(0)\right\|_{L^{\infty}} \leqslant C \varepsilon^{m}, \\
& \sup _{t \in[0, \min (\delta, T(\varepsilon))]}\left\|u_{a}^{\varepsilon}(t)-u^{\varepsilon}(t)\right\|_{L^{\infty}} \geqslant c \sqrt{\varepsilon} .
\end{aligned}
$$

Démonstration. - La preuve de cet énoncé est décomposée en quatre étapes. On part de la solution approchée $u_{a}^{\varepsilon}$ et on cherche une solution de $(\mathcal{S})$ sous la forme :

$$
u^{\varepsilon}=u_{a}^{\varepsilon}+\varepsilon^{m} \dot{u}^{\varepsilon}=\underline{u}+\sqrt{\varepsilon} u_{1}+\varepsilon v_{a}^{\varepsilon}+\varepsilon^{m} \dot{u}^{\varepsilon}, \quad m \geqslant 2 .
$$

Comme en (5.28), l'équation pour $\dot{u}^{\varepsilon}$ s'écrit :

$$
\mathcal{S}\left(u^{\varepsilon}, \partial_{t, x, \theta}\right) \dot{u}^{\varepsilon}+\mathcal{S}^{\prime}\left(t, x, \varepsilon^{m} \dot{u}^{\varepsilon}\right) \dot{u}^{\varepsilon}=f^{\varepsilon}=\mathrm{O}\left(\varepsilon^{\infty}\right) .
$$

On cherche une solution $\dot{u}^{\varepsilon}$ qui oscille selon les deux phases $\varphi$ et $\psi$, avec une durée de vie en $\mathrm{O}(\sqrt{\varepsilon}|\ln \varepsilon|)$ :

$$
\dot{u}^{\varepsilon}(t, x)=\mathbf{u}^{\varepsilon}\left(\frac{t}{\sqrt{\varepsilon}}, x, \frac{\varphi}{\varepsilon}, \frac{\psi}{\varepsilon}\right) .
$$

Les profils $\mathbf{u}^{\varepsilon}(s, x, \theta, \omega)$ sont périodiques en les variables rapides $\theta$ et $\omega$. L'équation pour $\mathbf{u}^{\varepsilon}$ s'écrit

$$
\mathbf{S}^{\varepsilon}\left(\varepsilon^{m} \mathbf{u}^{\varepsilon}, \partial_{s, x, \theta, \omega}\right) \mathbf{u}^{\varepsilon}=\sqrt{\varepsilon} f^{\varepsilon}=\mathrm{O}\left(\varepsilon^{\infty}\right)
$$

avec

$$
\begin{aligned}
\mathbf{S}^{\varepsilon}(\mathbf{v}, \partial):= & \Sigma_{0}\left(u_{a}^{\varepsilon}+\mathbf{v}\right) \partial_{s}+\sum_{j=1}^{d} \sqrt{\varepsilon} \Sigma_{j}\left(u_{a}^{\varepsilon}+\mathbf{v}\right) \partial_{x_{j}} \\
& +B+\frac{1}{\sqrt{\varepsilon}} S\left(u_{a}^{\varepsilon}+\mathbf{v} ; d \varphi \partial_{\theta}+d \psi \partial_{\omega}\right)+\sqrt{\varepsilon} C^{\varepsilon}(\mathbf{v})
\end{aligned}
$$

où

$$
B v:=(v \cdot \nabla \underline{S}(d \varphi)) \partial_{\theta} u_{1}
$$

et $C^{\varepsilon}(\mathbf{v})$ est une matrice dont les coefficients sont des fonctions $C^{\infty}$ de $\mathbf{v}$ et de $(s, x, \theta)$. Pour ne pas alourdir les notations, on désigne encore par $u_{a}^{\varepsilon}$ la fonction $u_{a}^{\varepsilon}(\sqrt{\varepsilon} s, x, \theta)$ (de même pour $v_{a}^{\varepsilon}$ et l'erreur $f^{\varepsilon}$ ).

Étape 1. Estimations sur le linéarisé. On se donne $\mathbf{v}$ et $\mathbf{f}$. On considère l'équation linéaire :

$$
\mathbf{S}^{\varepsilon}\left(\varepsilon^{m} \mathbf{v}, \partial_{s, x, \theta, \omega}\right) \mathbf{u}=\mathbf{f} .
$$

Proposition 6.1. - Étant donné $k>2+\frac{d}{2}$, il existe une constante $C_{0}$ (qui ne dépend que de $u_{a}^{\varepsilon}$ ) telle que pour tout $\left.\left.\varepsilon \in\right] 0,1\right]$, tout $\left.\left.T \in\right] 0, \varepsilon^{-1 / 2}\right]$ et tout

$$
\mathbf{v} \in C^{0}\left([0, T] ; H^{k}\right) \cap C^{1}\left([0, T] ; H^{k-1}\right)
$$

vérifiant

$$
\varepsilon^{m-\frac{1}{2}} \sup _{s \in[0, T]}\|\mathbf{v}(s)\|_{H^{k}}+\varepsilon^{m} \sup _{s \in[0, T]}\left\|\partial_{s} \mathbf{v}(s)\right\|_{H^{k-1}} \leqslant 1
$$


les solutions de (6.14) satisfont pour tout $s \in[0, T]$ :

$$
\|\mathbf{u}(s)\|_{H^{k}} \leqslant C_{0} e^{s C_{0}}\|\mathbf{u}(0)\|_{H^{k}}+C_{0} \int_{0}^{s} e^{\left(s-s^{\prime}\right) C_{0}}\left\|\mathbf{f}\left(s^{\prime}\right)\right\|_{H^{k}} d s^{\prime} .
$$

Étape 2. Construction BKW. On cherche une solution approchée de (6.12) sous la forme d'un développement asymptotique en $\varepsilon$ d'ordre fini :

$$
\mathbf{u}_{a}^{\varepsilon}=\sum_{n=0}^{\nu+1} \varepsilon^{n / 2} \mathbf{u}_{n}(s, x, \theta, \omega) \text {. }
$$

On note $\mathbf{r}^{\varepsilon}$ la quantité obtenue en reportant l'expression $\mathbf{u}_{a}^{\varepsilon}$ au niveau de l'équation (6.12) :

$$
\mathbf{r}^{\varepsilon}:=\mathbf{S}^{\varepsilon}\left(\varepsilon^{m} \mathbf{u}_{a}^{\varepsilon}, \partial_{s, x, \theta, \omega}\right) \mathbf{u}_{a}^{\varepsilon} .
$$

On initialise la série (6.17) par le terme amplificateur :

$$
\mathbf{u}_{0}(s, x, \theta, \omega)=e^{-\mu s} a(x)\left(w(\theta) e^{i \omega}+\overline{w(\theta)} e^{-i \omega}\right) .
$$

Ici $a \in C_{0}^{\infty}\left(\mathbb{R}^{d}\right)$ est à valeurs réelles et $w \in H^{\infty}(\mathbb{T})$ est une fonction propre de $\mathbb{G}_{1}$ associée à la valeur propre $\mu$, dont l'existence est garantie par l'hypothèse 6.1. On fixe l'entier $\nu$ de façon à ce que :

$$
\gamma_{0}\left(1+\frac{\nu}{2 m-1}\right)>C_{0}, \quad \nu \geqslant m
$$

Proposition 6.2. - Il existe des profils $\mathbf{u}_{n}$ pour $1 \leqslant n \leqslant \nu+1$, qui sont $C^{\infty}$ en $s \in[0, \infty[$, à valeurs dans $H^{\infty}\left(\mathbb{R}^{d} \times \mathbb{T}^{2}\right)$, et tels que :

(i) pour tout entier $k$, il existe une constante $D_{k}$ telle que pour tout $n$ compris entre 0 et $\nu$, et tout $s \in\left[0, \varepsilon^{-1 / 2}\right]$, on $a:$

$$
\sup _{j \leqslant k}\left\|\partial_{s}^{j} \mathbf{u}_{n}(s)\right\|_{H^{k-j}\left(\mathbb{R}^{d} \times \mathbb{T}^{2}\right)} \leqslant D_{k} e^{s \gamma_{0}\left(1+\frac{n}{2 m-1}\right)} .
$$

De plus, le terme $u_{\nu+1}$ se majore comme $u_{\nu}$;

(ii) avec $\mathbf{u}_{a}^{\varepsilon}$ défini par (6.17), pour tout $k$, il existe une constante $E_{k}$ telle que l'expression $\mathbf{r}^{\varepsilon}$ vérifie pour tout $s \in\left[0, \varepsilon^{-1 / 2}\right]:$

$$
\sup _{j \leqslant k}\left\|\partial_{s}^{j} \mathbf{r}^{\varepsilon}(s)\right\|_{H^{k-j}\left(\mathbb{R}^{d} \times \mathbb{T}^{2}\right)} \leqslant E_{k} \varepsilon^{\nu / 2} e^{s \gamma_{0}\left(1+\frac{\nu}{2 m-1}\right)} .
$$

On fixe désormais $k>2+\frac{d}{2}$. On pose :

$$
\rho(s):=\sqrt{\varepsilon} e^{s \gamma_{0} /(2 m-1)} .
$$

La proposition 6.2 garantit l'existence d'une constante $C_{1} \geqslant 1$ telle que pour tout $\left.\left.\varepsilon \in\right] 0,1\right]$ on a :

$$
\left\|\mathbf{u}_{a}^{\varepsilon}(s)\right\|_{H^{k}}+\left\|\partial_{s} \mathbf{u}_{a}^{\varepsilon}(s)\right\|_{H^{k-1}} \leqslant C_{1} e^{s \gamma_{0}}\left(1+\rho(s)^{\nu}\right)
$$

Étape 3. Estimations sur la solution exacte. On exprime la solution exacte de (6.12) sous la forme $\mathbf{u}^{\varepsilon}=\mathbf{u}_{a}^{\varepsilon}+\varepsilon^{\nu / 2} \mathbf{v}^{\varepsilon}$ avec $\mathbf{u}_{a}^{\varepsilon}$ donné par (6.17). L'équation pour $\mathbf{v}^{\varepsilon}$ se développe en :

$$
\mathbf{S}^{\varepsilon}\left(\varepsilon^{m}\left(\mathbf{u}_{a}^{\varepsilon}+\varepsilon^{\nu / 2} \mathbf{v}^{\varepsilon}\right), \partial\right) \mathbf{v}^{\varepsilon}+\varepsilon^{-\frac{\nu}{2}} \mathbf{S}^{\varepsilon}\left(\varepsilon^{m}\left(\mathbf{u}_{a}^{\varepsilon}+\varepsilon^{\nu / 2} \mathbf{v}^{\varepsilon}\right), \partial\right) \mathbf{u}_{a}^{\varepsilon}=\varepsilon^{\frac{1-\nu}{2}} f^{\varepsilon} .
$$


On décompose le terme source en :

$$
\begin{aligned}
\mathbf{h}^{\varepsilon}\left(\mathbf{v}^{\varepsilon}\right):= & \varepsilon^{\frac{1-\nu}{2}} f^{\varepsilon}-\varepsilon^{-\frac{\nu}{2}} \mathbf{S}^{\varepsilon}\left(\varepsilon^{m}\left(\mathbf{u}_{a}^{\varepsilon}+\varepsilon^{\nu / 2} \mathbf{v}^{\varepsilon}\right), \partial\right) \mathbf{u}_{a}^{\varepsilon}=\varepsilon^{\frac{1-\nu}{2}} f^{\varepsilon}-\varepsilon^{-\frac{\nu}{2}} \mathbf{r}^{\varepsilon} \\
& +\varepsilon^{m-\frac{1}{2}} \mathbf{D}_{1}^{\varepsilon}\left(\varepsilon^{m} \mathbf{u}_{a}^{\varepsilon}, \varepsilon^{m+\nu / 2} \mathbf{v}^{\varepsilon}\right)\left(\partial_{\theta, \omega} \mathbf{u}_{a}^{\varepsilon}, \mathbf{v}^{\varepsilon}\right) \\
& +\varepsilon^{m} \mathbf{D}_{2}^{\varepsilon}\left(\varepsilon^{m} \mathbf{u}_{a}^{\varepsilon}, \varepsilon^{m+\nu / 2} \mathbf{v}^{\varepsilon}\right)\left(\partial_{s, x} \mathbf{u}_{a}^{\varepsilon}, \mathbf{v}^{\varepsilon}\right) .
\end{aligned}
$$

Les $\mathbf{D}_{j}^{\varepsilon}(a, b)(\cdot, \cdot)$ pour $j=1$ ou 2 représentent des applications qui sont bilinéaires en les deux derniers arguments et dont les coefficients sont des fonctions $C^{\infty}$ de $(a, b)$ ainsi que des variables $(s, x, \theta, \omega)$. On considère :

$$
\mathbf{S}^{\varepsilon}\left(\varepsilon^{m}\left(\mathbf{u}_{a}^{\varepsilon}+\varepsilon^{\nu / 2} \mathbf{v}^{\varepsilon}\right), \partial\right) \mathbf{v}^{\varepsilon}=\mathbf{h}^{\varepsilon}\left(\mathbf{v}^{\varepsilon}\right) .
$$

Le système quasilinéaire (6.23) est hyperbolique symétrique. On sait que le problème de Cauchy pour (6.23) à données initiales nulles a une solution locale dans

$$
C^{0}\left(\left[0, T(\varepsilon)\left[; H^{k}\right) \cap C^{1}\left(\left[0, T(\varepsilon)\left[; H^{k-1}\right)\right.\right.\right.\right.
$$

pour un certain $T(\varepsilon)>0$.

Proposition 6.3. - Il existe une constante $C_{2} \geqslant 4 C_{1} \geqslant 4$ et un indice $\varepsilon_{0}>0$ tels que pour tout $\left.\varepsilon \in] 0, \varepsilon_{0}\right]$ on $a$ :

$$
T(\varepsilon)>T_{m}(\varepsilon):=\frac{1}{\gamma_{0}}\left(\left(m-\frac{1}{2}\right)|\ln \varepsilon|-\ln C_{2}\right)>0 .
$$

De plus, pour tout $s \in\left[0, T_{m}(\varepsilon)\right]$ la solution $\mathbf{v}^{\varepsilon}$ de (6.23) associée à la donnée initiale nulle vérifie:

$$
\left\|\mathbf{v}^{\varepsilon}(s)\right\|_{H^{k}}+\sqrt{\varepsilon}\left\|\partial_{s} \mathbf{v}^{\varepsilon}(s)\right\|_{H^{k-1}} \leqslant C_{2} e^{s \gamma_{0}\left(1+\frac{\nu}{2 m-1}\right)} .
$$

Étape 4. Conclusion. On suppose que les propositions 6.1, 6.2 et 6.3 sont démontrées. Soit $M \geqslant 1$. On examine la solution $\mathbf{u}^{\varepsilon}$ à l'instant :

$$
\bar{s}:=\frac{1}{\gamma_{0}}\left(\left(m-\frac{1}{2}\right)|\ln \varepsilon|-\ln \left(C_{2} M\right)\right) \leqslant T_{m}(\varepsilon) .
$$

Ce choix conduit à :

$$
C_{1} \varepsilon^{m-\frac{1}{2}} e^{\bar{s} \gamma_{0}} \leqslant C_{1} / C_{2} M \leqslant 1 / 4 M, \quad \rho(\bar{s})=\left(1 / C_{2} M\right)^{1 /(2 m-1)} \leqslant 1 .
$$

Avec (6.22), il vient :

$$
\varepsilon^{m-\frac{1}{2}}\left\|\mathbf{u}_{a}^{\varepsilon}(\bar{s})\right\|_{H^{k}}+\varepsilon^{m}\left\|\partial_{s} \mathbf{u}_{a}^{\varepsilon}(\bar{s})\right\|_{H^{k-1}} \leqslant 2 C_{1} / C_{2} M \leqslant 1 / 2 M .
$$

On a aussi :

$$
\varepsilon^{m}\left\|\mathbf{u}_{a}^{\varepsilon}(\bar{s})-\mathbf{u}_{0}(\bar{s})\right\|_{H^{k}} \leqslant \varepsilon^{m} C_{1} e^{\bar{s} \gamma_{0}}\left(\rho(\bar{s})+\rho(\bar{s})^{\nu}\right) \leqslant 2 C_{1} \sqrt{\varepsilon} \rho(\bar{s})^{2 m} .
$$

Au contraire, on a :

$$
\left\|\varepsilon^{m} \mathbf{u}_{0}(\bar{s})\right\|_{L^{\infty}} \geqslant c \varepsilon^{m} e^{\bar{s} \gamma_{0}} \geqslant c \sqrt{\varepsilon} \rho(\bar{s})^{2 m-1} .
$$

On en déduit que pour $M$ assez grand, on a :

$$
\left\|\varepsilon^{m} \mathbf{u}_{a}^{\varepsilon}(\bar{s})\right\|_{L^{\infty}} \geqslant \frac{c \sqrt{\varepsilon}}{2 C_{2} M} .
$$


L'estimation (6.25) montre que :

$$
\varepsilon^{m+\nu / 2}\left\|\mathbf{v}^{\varepsilon}(\bar{s})\right\|_{H^{k}} \leqslant C_{2} \varepsilon^{m} e^{\bar{s} \gamma_{0}} \rho(\bar{s})^{\nu} \leqslant C_{2} \sqrt{\varepsilon} \rho(\bar{s})^{2 m-1+\nu} .
$$

On retranche (6.29) à (6.27). Quitte à choisir $M$ assez grand, on obtient :

$$
\left\|\left(u^{\varepsilon}-u_{a}^{\varepsilon}\right)(\bar{s})\right\|_{L^{\infty}}=\left\|\varepsilon^{m} \mathbf{u}^{\varepsilon}(\bar{s})\right\|_{L^{\infty}} \geqslant \frac{c \sqrt{\varepsilon}}{4 C_{2} M} .
$$

Par ailleurs, on a :

$$
\left(u^{\varepsilon}-u_{a}^{\varepsilon}\right)(0)=\varepsilon^{m} \mathbf{u}^{\varepsilon}(0)=\varepsilon^{m} \mathbf{u}_{a}^{\varepsilon}(0), \quad\left\|\mathbf{u}_{a}^{\varepsilon}(0)\right\|_{L^{\infty}} \leqslant C .
$$

Le théorème 6.2 en résulte.

Il reste à démontrer les trois propositions ci-dessus. Les preuves sont classiques. Nous ne donnerons que les arguments essentiels.

Preuve de la proposition 6.1. - L'opérateur $\mathbf{S}^{\varepsilon}\left(\varepsilon^{m} \mathbf{v}, \partial\right)$ est de la forme

$$
\mathbf{S}^{\varepsilon}=\Sigma_{0}\left(u_{a}^{\varepsilon}+\varepsilon^{m} \mathbf{v}\right) \partial_{s}+\mathbf{R}^{\varepsilon}\left(\varepsilon^{m-\frac{1}{2}} \mathbf{v}, \partial_{x, \theta, \omega}\right)+\frac{1}{\sqrt{\varepsilon}} \underline{S}\left(d \varphi \partial_{\theta}+d \psi \partial_{\omega}\right)
$$

où $\mathbf{R}^{\varepsilon}$ est un système symétrique en $\partial_{x, \theta, \omega}$ dont les coefficients sont des fonctions $C^{\infty}$ des variables $(\sqrt{\varepsilon} s, x, \theta)$ et $\varepsilon^{m-\frac{1}{2}} \mathbf{v}$. En outre :

$$
\Sigma_{0}\left(u_{a}^{\varepsilon}+\varepsilon^{m} \mathbf{v}\right)=\underline{\Sigma}_{0}+\sqrt{\varepsilon} \mathbf{R}_{0}^{\varepsilon}\left(\varepsilon^{m-\frac{1}{2}} \mathbf{v}\right) .
$$

L'estimation (6.16) pour $k=0$ s'en déduit à l'aide d'une intégration par partie, en utilisant la symétrie de l'opérateur. La constante $C_{0}$ ne dépend que de bornes pour les normes $L^{\infty}$ des dérivées en $(x, \theta)$ des coefficients de $\mathbf{R}^{\varepsilon}\left(\varepsilon^{m-\frac{1}{2}} \mathbf{v}, \partial\right)$ et de la norme $L^{\infty}$ de $\partial_{s} \Sigma_{0}\left(u_{a}^{\varepsilon}+\varepsilon^{m} \mathbf{v}\right)$.

Pour obtenir les estimations $H^{k}$ on dérive l'équation (6.14) en $(x, \theta, \omega)$. Le point clé est que $\underline{\Sigma}_{0}$ et $\underline{S}\left(d \varphi \partial_{\theta}+d \psi \partial_{\omega}\right)$ commutent à ces dérivations. Le commutateur s'exprime donc à l'aide des dérivées en $(x, \theta, \omega)$ et de la dérivation $\sqrt{\varepsilon} \partial_{s}$ qui peut être échangée, via l'équation (6.14), en des dérivées selon $(x, \theta, \omega)$.

En utilisant les propriétés multiplicatives des espaces de Sobolev, comme au paragraphe 5.3, on obtient que, pour $k>2+\frac{d}{2}$, il existe une constante $C$ telle que, pour tout $\left.\left.\varepsilon \in\right] 0,1\right]$ et $\mathbf{v}$ vérifiant (6.15), on a pour $|\alpha| \leqslant k$ :

$$
\left\|\mathbf{S}^{\varepsilon}\left(\varepsilon^{m} \mathbf{v}, \partial\right) \partial_{x, \theta, \omega}^{\alpha} \mathbf{u}(s)\right\|_{L^{2}} \leqslant C\left(\|\mathbf{f}(s)\|_{H^{k}}+\|\mathbf{u}(s)\|_{H^{k}}\right) .
$$

Avec l'estimation $L^{2}$, la majoration (6.16) dans $H^{k}$ en résulte.

Preuve de la proposition 6.2. - En reportant (6.17) dans l'équation (6.12) on obtient une cascade de conditions. La première s'écrit :

$$
\underline{S}\left(d \varphi \partial_{\theta}+d \psi \partial_{\omega}\right) \mathbf{u}_{0}=0 .
$$

Pour $n \geqslant 0$, on trouve :

$$
\underline{S}\left(d \varphi \partial_{\theta}+d \psi \partial_{\omega}\right) \mathbf{u}_{n+1}+\left(\partial_{s}+G\right) \mathbf{u}_{n}=\mathbf{f}_{n-1}
$$

où $G$ est défini en (3.11) et $\mathbf{f}_{n-1}$ est une fonction de $\left(\mathbf{u}_{0}, \ldots, \mathbf{u}_{n-1}\right)$, somme de monômes

$$
\Phi \mathbf{u}_{n_{1}} \cdots \mathbf{u}_{n_{p-1}} \partial \mathbf{u}_{n_{p}} \quad \text { ou } \Phi \mathbf{u}_{n_{1}} \cdots \mathbf{u}_{n_{p}}
$$


dont les coefficients $\Phi$ sont $C^{\infty}$ bornés ansi que leurs dérivées pour $s \leqslant \varepsilon^{-1 / 2}$. De plus :

$$
\sup _{j} n_{j}<n \quad \text { et } \quad n_{1}+\cdots+n_{p} \leqslant n+1-2 m(p-1) .
$$

En particulier, $\mathbf{f}_{n-1}$ est linéaire en les $\mathbf{u}_{j}$ pour $n \leqslant 2 m-2$, au plus quadratique pour $n \leqslant 4 m-2$, etc.

On construit une solution de ces équations, partant de $\mathbf{u}_{0}$ donné par (6.18), en résolvant pour $n \geqslant 1$ :

$$
\left\{\begin{array}{l}
(\mathrm{Id}-\mathbb{P}) \mathbf{u}_{n}=\mathbb{Q}\left(\mathbf{f}_{n-2}-\left(\partial_{s}+G\right) \mathbf{u}_{n-1}\right), \\
\left(\partial_{s}+\mathbb{G}\right) \mathbb{P} \mathbf{u}_{n}=\mathbb{P}\left(\mathbf{f}_{n-1}-G(\operatorname{Id}-\mathbb{P}) \mathbf{u}_{n}\right), \\
\mathbb{P} \mathbf{u}_{n}=0 \quad \text { en } \quad s=0 .
\end{array}\right.
$$

Pour contrôler les $\mathbf{u}_{n}$, on a besoin de l'information suivante :

Lemme 6.2. - Soit $\beta \in \mathbb{Z}$ fixé. Pour tout $\gamma^{\prime}>\gamma_{0}$ et tout entier $k$, il existe une constante $C_{k}$ telle que l'on ait :

$$
\begin{aligned}
\left\|\mathbb{P}_{\beta} \mathbf{v}(s)\right\|_{H^{k}} \leqslant & C_{k} e^{\gamma^{\prime} s}\left\|\mathbb{P}_{\beta} \mathbf{v}(0)\right\|_{H^{k}} \\
& +C_{k} \int_{0}^{s} e^{\gamma^{\prime}\left(s-s^{\prime}\right)}\left\|\left(\partial_{s}+\mathbb{G}_{\beta}\right) \mathbf{v}\left(s^{\prime}\right)\right\|_{H^{k}} d s^{\prime} .
\end{aligned}
$$

Démonstration. - On raisonne par récurrence sur l'entier $k$.

Pour $k=0$, on utilise l'alinéa (ii) de l'hypothèse 6.1 :

$$
g_{\beta}(s) \leqslant g(s) \leqslant C e^{\gamma s}, \quad \forall \gamma>\gamma_{0} .
$$

L'estimation (6.32) pour $k=0$ s'en déduit directement.

On suppose maintenant (6.32) établie pour $0 \leqslant j<k$. On dérive l'équation à l'ordre $k$ et on met à profit l'estimation $L^{2}$ pour majorer les dérivées d'ordre $k$. Il faut faire attention aux commutateurs. L'opérateur $\mathbb{G}_{\beta}$ commute aux dérivations en $x$. Le projecteur $\mathbb{P}_{\beta}$ commute aux dérivations en $x$ et $\theta$. À l'ordre un, on a :

$$
\left[\partial_{\theta}, \mathbb{G}_{\beta}\right]=\mathbb{P}_{\beta}\left[\partial_{\theta}, G_{\beta}\right] \mathbb{P}_{\beta}=\mathbb{P}_{\beta}\left(i e^{i \theta} G_{\beta}^{+} \partial_{\theta}-i e^{-i \theta} G_{\beta}^{-} \partial_{\theta}\right) \mathbb{P}_{\beta}
$$

Ce commutateur est du même type que $\mathbb{G}_{\beta}$. On retrouve les expressions mises en jeu dans la preuve du lemme 6.1. Il s'ensuit que $\left[\partial_{\theta}, \mathbb{G}_{\beta}\right]$ est borné sur $H^{k}(\mathbb{T})$ pour tout $k$. Il en va de même pour les commutateurs d'ordre supérieur. On en déduit, avec $\left.\gamma^{\prime \prime} \in\right] \gamma_{0}, \gamma^{\prime}[$ :

$$
\begin{aligned}
\left\|\mathbb{P}_{\beta} \mathbf{v}(s)\right\|_{H^{k}} \leqslant & C_{k} e^{\gamma^{\prime \prime} s}\left\|\mathbb{P}_{\beta} \mathbf{v}(0)\right\|_{H^{k}} \\
& +C_{k} \int_{0}^{s} e^{\gamma^{\prime \prime}\left(s-s^{\prime}\right)}\left(\left\|\left(\partial_{s}+\mathbb{G}_{\beta}\right) \mathbf{v}\left(s^{\prime}\right)\right\|_{H^{k}}+\left\|\mathbb{P}_{\beta} \mathbf{v}\left(s^{\prime}\right)\right\|_{H^{k-1}}\right) d s^{\prime} .
\end{aligned}
$$

On applique l'hypothèse de récurrence pour contrôler la norme $H^{k-1} \operatorname{de~} \mathbb{P}_{\beta} \mathbf{v}\left(s^{\prime}\right)$. L'estimation souhaitée pour la norme $H^{k}$ suit.

Il s'agit maintenant d'établir l'estimation (6.20) pour les $\mathbf{u}_{n}$ obtenus via (6.31). On procède par récurrence sur l'entier $n$.

Pour $n=0$, l'inégalité (6.20) est une conséquence du choix de $\mathbf{u}_{0}$. En effet, la croissance de $\mathbf{u}_{0}$ et de ses dérivées en $s$ est contrôlée via l'alinéa (i) de l'hypothèse 6.1. 
Pour $0<n \leqslant 2 m-2$, le terme source $\mathbf{f}_{n-1}$ est linéaire en $\mathbf{u}_{j}$ avec $j<n$. Les seules harmoniques présentes en $\omega$ sont +1 et -1 de sorte que le système (6.31) se réduit à :

$$
\left\{\begin{array}{l}
\left(\mathrm{Id}-\mathbb{P}_{1}\right) \mathbf{u}_{n}=\mathbb{Q}_{1}\left(\mathbf{f}_{n-2}-\left(\partial_{s}+G_{1}\right) \mathbf{u}_{n-1}\right), \\
\left(\partial_{s}+\mathbb{G}_{1}\right) \mathbb{P}_{1} \mathbf{u}_{n}=\mathbb{P}_{1}\left(\mathbf{f}_{n-1}-G_{1}\left(\mathrm{Id}-\mathbb{P}_{1}\right) \mathbf{u}_{n}\right), \\
\mathbb{P}_{1} \mathbf{u}_{n}=0 \text { en } s=0 .
\end{array}\right.
$$

On suppose (6.20) vérifiée jusqu' au rang $n-1 \leqslant 2 m-3$. Cette hypothèse de récurrence et la forme de $\mathbf{f}_{n-2}$ garantissent l'existence d'une constante $C_{n, k}$ telle que pour $s \leqslant \varepsilon^{-1 / 2}$ :

$$
\sup _{j \leqslant k}\left\|\partial_{s}^{j} \mathbf{f}_{n-2}(s)-\partial_{s}^{j}\left(\partial_{s}+G\right) \mathbf{u}_{n-1}\right\|_{H^{k-j}\left(\mathbb{R}^{d} \times \mathbb{T}\right)} \leqslant C_{n, k} e^{s \gamma_{0}\left(1+\frac{n}{2 m-1}\right)}
$$

L'alinéa (iii) de l'hypothèse 6.1 implique que l'opérateur $\mathbb{Q}$ et donc $\mathbb{Q}_{1}$ est borné de $H^{k}(\mathbb{T})$ dans lui-même pour tout entier $k$. On voit ainsi que la composante $\left(\mathrm{Id}-\mathbb{P}_{1}\right) \mathbf{u}_{n}$ vérifie (6.20).

On se tourne vers l'équation concernant $\mathbb{P}_{1} \mathbf{u}_{n}$. Les renseignements obtenus ci-dessus indiquent que le terme source

$$
\mathbf{g}=\mathbb{P}_{1}\left(\mathbf{f}_{n-1}-G_{1}\left(\mathrm{Id}-\mathbb{P}_{1}\right) \mathbf{u}_{n}\right)
$$

vérifie (6.20). On met à profit le lemme 6.2 pour contrôler les dérivées en $(x, \theta)$ de $\mathbb{P}_{1} \mathbf{u}_{n}$. Par ailleurs, les dérivées en $s$ se récupèrent en utilisant l'équation

$$
\partial_{s} \mathbb{P}_{1} \mathbf{u}_{n}=-\mathbb{G}_{1} \mathbf{u}_{n}+\mathbf{g} .
$$

On se donne finalement $n \geqslant 2 m-1$. On suppose que l'estimation (6.20) est vraie jusqu'au rang $n-1$. Alors les monômes qui interviennent dans la constitution de $\mathbf{f}_{n-1}$ vérifient :

$$
\max \left(\left\|\Phi \mathbf{u}_{n_{1}} \cdots \mathbf{u}_{n_{p-1}} \partial \mathbf{u}_{n_{p}}(s)\right\|_{H^{k}} ;\left\|\Phi \mathbf{u}_{n_{1}} \cdots \mathbf{u}_{n_{p}}(s)\right\|_{H^{k}}\right) \leqslant C_{n, k} e^{s \gamma_{0} \sigma} .
$$

Ici $\sigma \leqslant 1+\frac{n}{2 m-1}$ si $p=1$. Pour $p \geqslant 2$, on obtient :

$$
\sigma \leqslant p+\frac{n_{1}+\cdots+n_{p}}{2 m-1} \leqslant p+\frac{n+1-2 m(p-1)}{2 m-1} \leqslant 1+\frac{n}{2 m-1} .
$$

On répète la démarche décrite ci-dessus pour estimer $(\mathrm{Id}-\mathbb{P}) u_{n}$ puis $\mathbb{P} u_{n}$. À cet endroit, il est important de tenir compte des harmoniques en $\omega$ qui apparaissent par interactions non linéaires. La difficulté soulevée tient à ce que les informations délivrées par les lemmes 6.1 et 6.2 ne sont pas uniformes en $\beta$. Toutefois, comme $n \leqslant \nu+1$ avec $\nu$ fixé, le nombre des harmoniques créées reste fini. Du coup les arguments précédents se transposent tels quels.

L'inégalité (6.20) est prouvée pour tout $n$ avec $0 \leqslant n \leqslant \nu$. Le terme de correction $u_{\nu+1}$ se traite à part. On prend $u_{\nu+1}=(\mathrm{Id}-\mathbb{P}) u_{\nu+1}$ identifié à l'aide de la première ligne de $(6.31)$. On retrouve ainsi sur $u_{\nu+1}$ le contrôle dont on dispose sur $u_{\nu}$.

L'estimation (6.21) s'obtient par un examen du reste $\mathbf{r}^{\varepsilon}$. On voit que $\mathbf{r}^{\varepsilon}$ comporte $\varepsilon^{\nu / 2}$ en facteur et met en jeu les dérivées des $u_{n}$ pour $0 \leqslant n \leqslant \nu+1$. Il suffit alors d'utiliser (6.20).

Preuve de la proposition 6.3. - On résout l'équation (6.23) à l'aide du schéma itératif :

$$
\mathbf{S}^{\varepsilon}\left(\varepsilon^{m}\left(\mathbf{u}_{a}^{\varepsilon}+\varepsilon^{\nu / 2} \mathbf{v}_{n}^{\varepsilon}\right), \partial\right) \mathbf{v}_{n+1}^{\varepsilon}=\mathbf{h}^{\varepsilon}\left(\mathbf{v}_{n}^{\varepsilon}\right), \quad \mathbf{v}_{n+1 \mid s=0}^{\varepsilon}=0 .
$$

On initialise ce schéma avec $\mathbf{v}_{0}^{\varepsilon}=0$. La proposition 6.2 implique qu'il existe une constante $K_{0}$ telle que pour $s \leqslant \varepsilon^{-1 / 2}$ on ait :

$$
\left\|\varepsilon^{\frac{1-\nu}{2}} f^{\varepsilon}-\varepsilon^{\frac{-\nu}{2}} \mathbf{r}^{\varepsilon}(s)\right\|_{H^{k}\left(\mathbb{R}^{d} \times \mathbb{T}^{2}\right)} \leqslant K_{0} e^{s \gamma_{1}}, \quad \gamma_{1}:=\gamma_{0}\left(1+\frac{\nu}{2 m-1}\right) .
$$


On montre par récurrence sur $n$ qu'il existe un indice $\varepsilon_{0}$ et une constante $K \geqslant 4 C_{1}$ tels que pour $\left.\varepsilon \in] 0, \varepsilon_{0}\right], n \geqslant 0$ et

$$
0 \leqslant s \leqslant T_{m}(\varepsilon):=\frac{1}{\gamma_{0}}\left(\left(m-\frac{1}{2}\right)|\ln \varepsilon|-\ln K\right)
$$

on ait :

$$
\left\|\mathbf{v}_{n}^{\varepsilon}(s)\right\|_{H^{k}}+\sqrt{\varepsilon}\left\|\partial_{s} \mathbf{v}_{n}^{\varepsilon}(s)\right\|_{H^{k-1}} \leqslant K e^{s \gamma_{1}} .
$$

La proposition 6.3 écrite avec $C_{2}=K$ s'en déduit. En effet, à $\varepsilon$ fixé, on sait que la suite $\mathbf{v}_{n}^{\varepsilon}$ converge dans $C^{0}\left([0, T] ; H^{k-1}\right)$ vers la solution de (6.23) sur tout intervalle de temps où elle est bornée dans $C^{0}\left([0, T] ; H^{k}\right)$ et dans $C^{1}\left([0, T] ; H^{k-1}\right)$. Les estimations (6.38) qui sont uniformes en $n$ montrent que la suite $\left\{\mathbf{v}_{n}^{\varepsilon}\right\}_{n \in \mathbb{N}}$ converge au moins sur $\left[0, T_{m}(\varepsilon)\right]$. La solution de (6.23) est donc définie au moins sur cet intervalle et les majorations (6.38) impliquent que la limite $\mathbf{v}^{\varepsilon}$ vérifie (6.25).

Il reste finalement à démontrer les majorations (6.38). Pour $n=0$, elles sont triviales. Supposons qu'elles sont vérifiées au rang $n$. On remarque d'abord que, pour $s \leqslant T_{m}(\varepsilon)$, le contrôle (6.38) et l'inégalité $\nu \geqslant m$ impliquent :

$$
\varepsilon^{m-\frac{1}{2}}\left\|\varepsilon^{\frac{\nu}{2}} \mathbf{v}_{n}^{\varepsilon}(s)\right\|_{H^{k}}+\varepsilon^{m}\left\|\varepsilon^{\frac{\nu}{2}} \partial_{s} \mathbf{v}_{n}^{\varepsilon}(s)\right\|_{H^{k-1}} \leqslant K^{-\frac{\nu}{2 m-1}} \leqslant 1 / 2 .
$$

Par ailleurs, la proposition 6.2 se traduit par l'inégalité (6.26) qui pour $M=1$ donne :

$$
\varepsilon^{m-\frac{1}{2}}\left\|\mathbf{u}_{a}^{\varepsilon}(s)\right\|_{H^{k}}+\varepsilon^{m}\left\|\partial_{s} \mathbf{u}_{a}^{\varepsilon}(s)\right\|_{H^{k-1}} \leqslant 2 C_{1} / K \leqslant 1 / 2 .
$$

On exploite ces renseignements pour estimer le membre de droite de (6.23). D'abord on a :

$$
\left\|\mathbf{D}_{j}^{\varepsilon}\left(\varepsilon^{m} \mathbf{u}_{a}^{\varepsilon}, \varepsilon^{m+\nu / 2} \mathbf{v}_{n}^{\varepsilon}\right)\right\|_{H^{k}} \leqslant C_{4} .
$$

On en déduit :

$$
\begin{aligned}
& \left\|\varepsilon^{m-\frac{1}{2}} \mathbf{D}_{1}^{\varepsilon}\left(\varepsilon^{m} \mathbf{u}_{a}^{\varepsilon}, \varepsilon^{m+\nu / 2} \mathbf{v}_{n}^{\varepsilon}\right)\left(\partial_{\theta, \omega} \mathbf{u}_{a}^{\varepsilon}, \mathbf{v}_{n}^{\varepsilon}\right)\right\|_{H^{k}} \leqslant C_{5} K^{-\frac{\nu}{2 m-1}} e^{\gamma_{1} s} \\
& \left\|\varepsilon^{m} \mathbf{D}_{2}^{\varepsilon}\left(\varepsilon^{m} \mathbf{u}_{a}^{\varepsilon}, \varepsilon^{m+\nu / 2} \mathbf{v}_{n}^{\varepsilon}\right)\left(\partial_{s, x} \mathbf{u}_{a}^{\varepsilon}, \mathbf{v}_{n}^{\varepsilon}\right)\right\|_{H^{k}} \leqslant C_{5} K^{-\frac{\nu}{2 m-1}} e^{\gamma_{1} s}
\end{aligned}
$$

Et finalement :

$$
\left\|\mathbf{h}^{\varepsilon}\left(\mathbf{v}_{n}^{\varepsilon}\right)\right\|_{H^{k}} \leqslant\left(K_{0}+2 C_{5} K^{-\frac{\nu}{2 m-1}}\right) e^{\gamma_{1} s} .
$$

Ici les constantes $C_{4}$ et $C_{5}$ sont indépendantes de $K$, $\varepsilon$ et $n$. On ajoute (6.39) et (6.40) pour retenir :

$$
\varepsilon^{m-\frac{1}{2}}\left\|\mathbf{u}_{a}^{\varepsilon}(s)+\varepsilon^{\frac{\nu}{2}} \mathbf{v}_{n}^{\varepsilon}(s)\right\|_{H^{k}}+\varepsilon^{m}\left\|\partial_{s} \mathbf{u}_{a}^{\varepsilon}(s)+\varepsilon^{\frac{\nu}{2}} \partial_{s} \mathbf{v}_{n}^{\varepsilon}(s)\right\|_{H^{k-1}} \leqslant 1 .
$$

Dès lors, on peut appliquer la proposition 6.1 sur (6.35) qui livre :

$$
\left\|\mathbf{v}_{n+1}^{\varepsilon}(s)\right\|_{H^{k}} \leqslant C_{0}\left(K_{0}+2 C_{5} K^{-\frac{\nu}{2 m-1}}\right) \int_{0}^{s} e^{C_{0}\left(s-s^{\prime}\right)} e^{\gamma_{1} s^{\prime}} d s^{\prime} .
$$

L'entier $\nu$ a été ajusté en (6.19) de manière à ce que $C_{0}<\gamma_{1}$. Du coup :

$$
\left\|\mathbf{v}_{n+1}^{\varepsilon}(s)\right\|_{H^{k}} \leqslant C_{0}\left(K_{0}+2 C_{5} K^{-\frac{\nu}{2 m-1}}\right) e^{\gamma_{1} s} /\left(\gamma_{1}-C_{0}\right) .
$$

On exprime $\sqrt{\varepsilon} \partial_{s} \mathbf{v}_{n+1}^{\varepsilon}(s)$ à l'aide de l'équation (6.35). La forme de l'opérateur $\mathbf{S}^{\varepsilon}$ et le contrôle (6.41) fournissent :

$$
\sqrt{\varepsilon}\left\|\partial_{s} \mathbf{v}_{n+1}^{\varepsilon}(s)\right\|_{H^{k-1}} \leqslant C_{6}\left(\left\|\mathbf{v}_{n+1}^{\varepsilon}(s)\right\|_{H^{k}}+\sqrt{\varepsilon}\left\|\mathbf{h}^{\varepsilon}\left(\mathbf{v}_{n}^{\varepsilon}(s)\right)\right\|_{H^{k-1}}\right) .
$$


Pour pouvoir conclure, il faut que :

$$
\left(\left(1+C_{6}\right) C_{0} /\left(\gamma_{1}-C_{0}\right)+C_{6} \sqrt{\varepsilon}\right)\left(K_{0}+2 C_{5} K^{-\frac{\nu}{2 m-1}}\right) \leqslant K
$$

Il suffit de prendre $\varepsilon_{0}$ assez petit et $K$ assez grand pour que cette inégalité soit vraie. La démonstration de la proposition 6.3 et par conséquent celle du théorème 6.2 en découle.

\section{RÉFÉRENCES}

[1] Artola M., Majda A., Nonlinear development of instabilities in supersonic vortex sheets I. The basic kink mode, Physica D 28 (1987) 253-281.

[2] Boillat G., Symétrisation des systèmes d'e.d.p. avec densité d'énergie convexe et contraintes, CRAS Sér. I 295 (1982) 551-554.

[3] Browning G., Kreiss H.O., Problems with different time scales for non-linear partial differential equations, SIAM J. Appl. Math. 42 (1982) 704-718.

[4] Cheverry C., GuÈs O., MÉtivier G., Oscillations de grande amplitude, En préparation.

[5] Colin T., LAnnes D., Long-wave short-wave resonance for nonlinear geometric optics, Duke Math. J. 107 (2) (2001) 351-419.

[6] Corli A., GuÈs O., Stratified solutions for systems of conservation laws, Trans. Amer. Math. Soc. 353 (6) (2001) 2459-2486.

[7] E W., Propagation of oscillations in the solutions of $1-D$ compressible fluid equations, Com. PDE 17 (3/4) (1992) 347-370.

[8] Friedrichs K.-O., LAX P., Systems of conservation laws with a convex extension, Proc. Nat. Acad. Sci. USA 68 (1971) 1686-1688.

[9] Friedlander S., Strauss W., Vishik M., Nonlinear instability in an ideal fluid, Ann. Inst. Henri Poincaré 14 (2) (1997) 187-209.

[10] Grenier E., On the nonlinear instability of Euler and Prandtl equations, Comm. Pure Appl. Math. 53 (9) (2000) 1067-1091.

[11] GUÈs O., Ondes multidimensionnelles $\varepsilon$-stratifiées et oscillations, Duke Math. J. 68 (3) (1992) 401446.

[12] GuÈs O., Développement asymptotique de solutions exactes de systèmes hyperboliques quasilinéaires, Asymptotic Anal. 6 (3) (1993) 241-269.

[13] Heibig A., Error estimates for oscillatory solutions to hyperbolic systems of conservation laws, Com. PDE 18 (1993) 281-304.

[14] JEANNE P.-Y., Optique géométrique pour des systèmes semi-linéaires avec invariance de jauge, Soumis pour publication aux Mémoires de la SMF.

[15] Joly J.-L., Métivier G., Rauch J., Optique géométrique non linéaire et équations de Maxwell Bloch, Prépublication de l'École Polytechnique, exposé XI, 1999.

[16] Joly J.-L., Métivier G., RAUCH J., Justification of multidimensional single phase semilinear geometric optics, Trans. Amer. Math. Soc. 330 (2) (1992) 599-623.

[17] Joly J.-L., MÉtivier G., RAUCH J., Recent results in non-linear geometric optics, in: Hyperbolic Problems: Theory, Numerics, Applications, vol. II (Zürich, 1998), in: Internat. Ser. Numer. Math., vol. 130, Birkhäuser, Basel, 1999, pp. 723-736.

[18] Joly J.-L., Métivier G., Rauch J., Coherent and focusing multidimensional nonlinear geometric optics, Ann. Sci. Éc. Norm. Sup. (4) 28 (1) (1995) 51-113.

[19] Majda A., Compressible Fluid Flow and Systems of Conservation Laws in Several Space Variables, Springer-Verlag, New York, 1984.

[20] MuseuX A., Problème de Cauchy pour des solutions stratifiées de systèmes hyperboliques de lois de conservation, Thèse université de Nice 2002 et Prépublication 616 du labo. Dieudonné, 2001.

[21] Schochet S., Fast singular limits of hyperbolic PDEs, J. Differential Eqs. 114 (1994) 476-512.

[22] SERre D., Oscillations non linéaires de haute fréquence. Dim $\geqslant 2$, in: Marino A., Murthy M.K.V. (Eds.), Nonlinear Variational Problems and Partial Differential Equations, in: Pitman Res. Notes in Math., vol. 320, Longman, London, 1995, pp. 245-294 ; Springer-Verlag, New York, 1983. 
[23] SERRE D., Quelques méthodes d'étude de la propagation d'oscillations hyperboliques non linéaires, in : Séminaire EDP 1990-91, École Polytechnique, exposé no XX.

[24] SÉvenNeC B., Géométrie des systèmes hyperboliques de lois de conservation, Société mathématique de France, Mémoire 56, Suppl. Bull. Soc. Math. France 122 (1) (1994).

(Manuscrit reçu le 23 mai 2002; accepté, après révision, le 24 janvier 2003.)

Christophe CHEVERrY

Institut Girard Désargues,

Université de Lyon I,

Bâtiment 101,

43 bld du 11 novembre 1918 ,

69622 Villeurbanne Cedex, France

E-mail : cheverry@igd.univ-lyon1.fr

Olivier GuÈs

Département de Mathématiques,

Université de Nice-Sophia Antipolis,

Parc Valrose,

06108 Nice Cedex 2, France

\section{Guy MÉTIVIER} IRMAR,

Campus de Beaulieu,

Université de Rennes I,

Campus de Beaulieu,

35402 Rennes Cedex, France

E-mail : guy.metivier@univ-rennes1.fr 